\title{
VISCOUS APPROXIMATION OF QUASISTATIC EVOLUTIONS FOR A COUPLED ELASTOPLASTIC-DAMAGE MODEL
}

\author{
VITO CRISMALE AND GIULIANO LAZZARONI
}

\begin{abstract}
Employing the technique of vanishing viscosity and time rescaling, we show the existence of quasistatic evolutions for elastoplastic materials with incomplete damage affecting both the elastic tensor and the plastic yield surface, in a softening framework and in small strain assumptions.
\end{abstract}

Keywords: variational models, quasistatic evolution, vanishing viscosity, elastoplasticity, damage models, incomplete damage, softening.

2010 Mathematics Subject Classification: 74C05, 74R05, 74G65, 35Q74, 49J45.

\section{Contents}

$\begin{array}{lr}\text { Introduction } & 1 \\ \text { 1. Mechanical assumptions and mathematical tools } & 5 \\ \text { 2. Discrete-time viscous approximation } & 10 \\ \text { 3. Viscous evolutions } & 18 \\ \text { 4. Rescaled quasistatic viscosity evolutions } & 26 \\ \text { 5. Properties of rescaled quasistatic viscosity evolutions } & 31 \\ \text { Appendix A. Auxiliary results } & 35 \\ \text { References } & 37\end{array}$

\section{INTRODUCTION}

Materials are subject to irreversible processes resulting in inelastic behaviour, such as damage and plasticity. Damage, originated by microcracks and microvoids, affects the elastic response of the material with respect to loading and unloading, whilst plasticity produces residual deformations that remain after complete unloading. Combining these two models provides a better description of e.g. cyclic loading, see for instance [15, Section 3.6] or [22, Section 7.5].

Gradient damage models are characterized by a dependence of the elastic modulus on a scalar internal variable that describes the damage state of the material; indeed, the stored elastic energy decreases as damage increases [13]. To localize damage, the energy is assumed to contain a term depending on the derivatives of the damage variable. We consider incomplete damage only, i.e., during the process the material keeps some elastic properties. We adopt the softening framework of [1, 2], thus damage is coupled with plasticity by requiring that the plastic yield surface shrinks when damage increases; hence the plastic dissipation depends on the damage variable.

The existence of quasistatic evolutions for a coupled elastoplastic-damage model was proved in [4] using the ansatz of global stability; more precisely, at any time instant the configuration is assumed to be a global minimizer of the sum of the stored elastic energy and of the plastic dissipation. This approach, based on the so-called energetic formulation of rate-independent processes (see [24] and references therein), was followed e.g. in $[11,27,38]$ for damage evolution, in $[5,6]$ for plasticity, and in $[29,30]$ for delamination problems with damage and plasticity at the interface.

However, the concept of globally-stable evolution is not satisfactory from the physical point of view: indeed, due the nonconvexity of the total energy, the solution is forced to develop jumps in time between the energy wells, overtaking energy barriers. To avoid the drawbacks of global minimality, in this paper we adopt a vanishing viscosity approach, i.e., we obtain a quasistatic evolution as a limit of solutions to some rate-dependent systems containing a viscous dissipation that tends to zero. Moreover, we characterize the jumps in time of the limit evolution by means of a suitable time-reparametrization; here we follow a technique first proposed in [10], then refined in [25, 26, 28], and used e.g. in [18, 17] for damage, in [7, 8] for plasticity, and in $[16,21]$ for brittle fracture.

Preprint SISSA 05/2015/MATE. 
Description of the model. The formulation of the model is essentially the same as in [4] and follows [13] for modeling damage and [5] for plasticity.

We study the evolution of damage and plasticity in an elastoplastic body whose reference configuration is a bounded, Lipschitz, open set $\Omega \subset \mathbb{R}^{n}, n \geq 2$. Starting from a given initial condition, the evolutionary process in a fixed time interval $[0, T]$ is driven by a (quasistatic) time-dependent loading; for simplicity we limit it to a prescribed displacement imposed on the Dirichlet part of the boundary $\partial_{D} \Omega \subset \partial \Omega$ and given by the trace on $\partial_{D} \Omega$ of a function $w \in H^{1}\left(\mathbb{R}^{n} ; \mathbb{R}^{n}\right)$. The remainder of the boundary $\partial_{N} \Omega$ is traction free, and no body forces are present. Under the assumption of small strain, the linearized strain $E u$ (i.e., the symmetric part of the spatial gradient of the displacement $u$ ) is additively decomposed in an elastic and a (deviatoric) plastic part, denoted by $e$ and $p$, respectively.

For every boundary datum $w \in H^{1}\left(\mathbb{R}^{n} ; \mathbb{R}^{n}\right)$, we define $A(w)$, the set of admissible displacements and strains, as the set of all triples $(u, e, p)$ with $u \in B D(\Omega)$ a function of bounded deformation, $e \in$ $L^{2}\left(\Omega ; \mathbb{M}_{s y m}^{n \times n}\right)$ a function valued in the symmetric matrices, and $p \in M_{b}\left(\Omega \cup \partial_{D} \Omega ; \mathbb{M}_{D}^{n \times n}\right)$ a bounded Borel measure valued in the trace-free matrices, such that

$$
E u=e+p \text { in } \Omega \text { and } p=(w-u) \odot \nu \mathcal{H}^{n-1} \text { on } \partial_{D} \Omega,
$$

where the latter equation is understood in the sense of traces and $\mathcal{H}^{n-1}$ is the $(n-1)$-dimensional Hausdorff measure.

Damage is described by an internal variable $\alpha$, defined on $[0, T] \times \Omega$ and with values into $[0,1]$, with $\alpha=1$ denoting the sound material and $\alpha=0$ the most damaged state. The stored elastic energy depends on $\alpha$ and is defined by $\mathcal{Q}:=\frac{1}{2}\langle\mathbb{C}(\alpha) e, e\rangle_{L^{2}}$, with $e \mapsto \mathbb{C}(\alpha) e: e$ nondecreasing in $\alpha$ for every $e$ and equicoercive with respect to $\alpha$ (indeed, damage is incomplete by assumption). The dissipation relative to the damage process is given by a term $D(\alpha):=\int_{\Omega} d(\alpha) \mathrm{d} x$, with $d$ sufficiently regular and diverging to $+\infty$ at zero with a certain rate, so that the state $\alpha=0$ is never reached during the evolution; this corresponds to the hypothesis of incomplete damage.

We introduce a regularizing term

$$
\frac{\kappa}{2}|\alpha|_{m, 2}^{2}=\frac{\kappa}{2} \sum_{|\beta|=m}\left\|D^{\beta} \alpha\right\|_{2}^{2}
$$

with $m:=\left[\frac{n}{2}\right]+1$ and $\kappa$ a positive constant, that can be regarded as a factor of influence of damage (see e.g. [38]). An analogous regularization was used in [18] with $m=n / 2$; here we need $m>n / 2$ to obtain compact embedding in $C(\bar{\Omega})$ and thus the lower semicontinuity of plastic dissipation by Reshetnyak's theorem. Another possible choice for the regularizing term would be $\|\nabla \alpha\|_{\gamma}^{\gamma}$ with $\gamma>n$, used e.g. in [4]; however, in the setting of vanishing viscosity this choice does not allow to get an energy equality, as shown in [17]. We refer to Remark 2.10 below for other comments on the regularization. The total mechanical energy is then

$$
\mathcal{E}(\alpha, e):=\mathcal{Q}(\alpha, e)+D(\alpha)+\frac{\kappa}{2}|\alpha|_{m, 2}^{2}
$$

In order to introduce the plasticity in the model, we consider a family $(K(\alpha))_{\alpha \in[0,1]}$ of convex and compact subsets of $\mathbb{M}_{D}^{n \times n}$. Their dependence on $\alpha$ is assumed to be Lipschitz in $\alpha$ with respect to the Hausdorff distance and such that

$$
B_{r}(0) \subset K(0) \subset K\left(\alpha_{1}\right) \subset K\left(\alpha_{2}\right) \subset K(1) \subset B_{R}(0),
$$

for every $\alpha_{1} \leq \alpha_{2}$, where $0<r<R$ are constant. These sets are called constraint sets, since the deviatoric part of the stress must belong to $K(\alpha(t, x))$ for every $t$ and a.e. $x$. Hence, the support function of $K(\alpha)$, that is

$$
H(\alpha, \xi):=\sup _{\sigma \in K(\alpha)} \sigma: \xi
$$

is convex and positively one-homogeneous in $\xi$, and Lipschitz in $\alpha$ uniformly with respect to $\xi$ whenever $|\xi|=1$. In some cases, in addition to the assumptions above, we require that $H$ is differentiable in $\alpha$, that $\partial_{\alpha} H$ is Lipschitz in $\alpha$, and that $\xi \mapsto H\left(\alpha_{2}, \xi\right)-H\left(\alpha_{1}, \xi\right)$ is convex for every $\alpha_{1} \leq \alpha_{2}$.

Then for every $p \in M_{b}\left(\Omega \cup \partial_{D} \Omega ; \mathbb{M}_{D}^{n \times n}\right)$ we define the plastic potential as

$$
\mathcal{H}(\alpha, p):=\int_{\Omega \cup \partial_{D} \Omega} H\left(\alpha(x), \frac{\mathrm{d} p}{\mathrm{~d}|p|}(x)\right) \mathrm{d}|p|(x),
$$

where $p /|p|$ is the Radon-Nikodym derivative of the measure $p$ with respect to its variation $|p|$. Given $\alpha:[0, T] \rightarrow C(\bar{\Omega})$ and $p:[0, T] \rightarrow M_{b}\left(\Omega \cup \partial_{D} \Omega ; \mathbb{M}_{D}^{n \times n}\right)$, the plastic dissipation in a time interval $[s, t]$ is given by

$$
\mathcal{V}_{\mathcal{H}}(\alpha, p ; s, t):=\sup \left\{\sum_{j=1}^{N} \mathcal{H}\left(\alpha\left(t_{j}\right), p\left(t_{j}\right)-p\left(t_{j-1}\right)\right): s=t_{0}<t_{1}<\cdots<t_{N}=t, N \in \mathbb{N}\right\} .
$$


We use the fact, proved in [4], that

$$
\mathcal{V}_{\mathcal{H}}(\alpha, p ; s, t)=\int_{s}^{t} \mathcal{H}(\alpha(\tau), \dot{p}(\tau)) \mathrm{d} \tau
$$

whenever $\alpha$ is nondecreasing in time, $\alpha(t)$ is uniformly bounded in $H^{m}(\Omega)$, and $p$ is absolutely continuous from $[0, T]$ into $M_{b}\left(\Omega \cup \partial_{D} \Omega ; \mathbb{M}_{D}^{n \times n}\right)$. To ease the reading, when $\alpha \in C(\bar{\Omega} ;[0,1])$ does not depend on time we use the symbol $\widehat{\mathcal{V}}_{H}$ instead of $\mathcal{V}_{H}$, hence

$$
\widehat{\mathcal{V}}_{\mathcal{H}}(\alpha, p ; s, t)=\int_{s}^{t} \mathcal{H}(\alpha, \dot{p}(\tau)) \mathrm{d} \tau \text {. }
$$

Finally we introduce the "generalized energy"

$$
\mathcal{E}_{\lambda}(\alpha, e ; p, t):=\mathcal{E}(\alpha, e)+\lambda \widehat{\mathcal{V}}_{\mathcal{H}}(\alpha, p ; 0, t)
$$

with $\lambda \in[0,1]$. Its meaning will be discussed in the final part of this introduction.

Viscous approximation. To prove the existence of a quasistatic evolution for the coupled elastoplasticdamage model, we first add to the system a viscous term, driven by a small parameter $\varepsilon>0$. Hence, for given $\varepsilon$ we construct some approximate viscous evolutions by discrete-time approximation. More precisely, for every $k \in \mathbb{N}$ we set $\tau_{k}:=T / k$ and define inductively $\left(\alpha_{k}^{i}, u_{k}^{i}, e_{k}^{i}, p_{k}^{i}\right)$ as a solution to the incremental minimum problem

$$
\min _{\substack{0 \leq \beta \leq \alpha_{k}^{i-1} \\(u, \eta, q) \in A\left(w_{k}^{i}\right)}}\left\{\mathcal{E}_{\lambda}\left(\beta, \eta ; p_{k}, t_{k}^{i-1}\right)+\mathcal{H}\left(\beta, q-p_{k}^{i-1}\right)+\frac{\varepsilon}{2 \tau_{k}}\left\|\beta-\alpha_{k}^{i-1}\right\|_{L^{2}(\Omega)}^{2}\right\}
$$

where $\left(\alpha_{k}^{0}, u_{k}^{0}, e_{k}^{0}, p_{k}^{0}\right):=\left(\alpha_{0}, u_{0}, e_{0}, p_{0}\right)$ is the (sufficiently regular) initial condition, $w_{k}^{i}:=w\left(t_{k}^{i}\right)$ for $i \in\{1, \ldots, k\}$ is the updated boundary loading, and

$$
p_{k}(t):=p_{k}^{j}+\frac{t-t_{k}^{j}}{\tau_{k}}\left(p_{k}^{j+1}-p_{k}^{j}\right) \quad \text { for } t \in\left[t_{k}^{j}, t_{k}^{j+1}\right) \text { and } j=0, \ldots, k-1
$$

is the piecewise affine interpolant of the solutions $p_{k}^{i}$ to problem (0.1) itself. (Notice that the term $\mathcal{E}_{\lambda}\left(\beta, \eta ; p_{k}, t_{k}^{i-1}\right)$ occurring in $(0.1)$ only depends on $p_{k}^{0}, \ldots, p_{k}^{i-1}$, so the problem is well posed.) We observe that, if one sets $\varepsilon=0$, then (0.1) reduces to the incremental global minimum problem used in [4], so one obtains the energetic formulation in the limit as $k \rightarrow \infty$. Instead, passing to the limit first as $k \rightarrow \infty$ and then for $\varepsilon \rightarrow 0$ allows one to select stationary points that are not necessarily global minimizers.

Hence, we consider the piecewise affine interpolants $\alpha_{k}, u_{k}, e_{k}, p_{k}$ of the functions $\alpha_{k}^{i}, u_{k}^{i}, e_{k}^{i}, p_{k}^{i}$ and find suitable a priori estimates to pass to the limit as $k \rightarrow \infty$. A remarkable fact is that, even if we could expect that $\alpha_{k} \in H^{1}\left(0, T ; L^{2}(\Omega)\right)$ with $\int_{0}^{T}\left\|\dot{\alpha}_{k}(s)\right\|_{L^{2}(\Omega)}^{2} \leq C_{\varepsilon}$ since we take in $(0.1)$ an $L^{2}$-viscosity term, we actually get that $\alpha_{k} \in H^{1}\left(0, T ; H^{m}(\Omega)\right)$ with

$$
\int_{0}^{T}\left\|\dot{\alpha}_{k}(s)\right\|_{H^{m}(\Omega)}^{2} \leq C_{\varepsilon} \quad \text { and } \quad \int_{0}^{T}\left\|\dot{\alpha}_{k}(s)\right\|_{H^{m}(\Omega)} \leq C
$$

for $k$ large. These estimates, contained in Propositions 2.7 and 2.8, rely on analogous properties proved in [18] for damage without plasticity; in our context a key tool is the inequality

$$
\left\|\dot{e}_{k}(t)\right\|_{2}+\left\|\dot{p}_{k}(t)\right\|_{1}+\left\|E \dot{u}_{k}(t)\right\|_{1} \leq C\left(\left\|\dot{\alpha}_{k}(t)\right\|_{\infty}+\left\|E \dot{w}_{k}(t)\right\|_{2}\right),
$$

obtained adapting arguments from [9, 34], see also [20] for related estimates in the study of sweeping processes.

The first estimate in (0.2) allows us to pass to the limit as $k \rightarrow \infty$ and show the existence of $\varepsilon$ approximate viscous evolutions, namely quadruples of absolutely continuous functions $\left(\alpha_{\varepsilon}, u_{\varepsilon}, e_{\varepsilon}, p_{\varepsilon}\right)$ satisfying an irreversibility condition for the damage variable, an energy balance, and a first order stability condition of Kuhn-Tucker type (see Definition 3.1 and Theorem 3.7). From the second estimate in (0.2) and from (0.3) we obtain that

$$
t+\int_{0}^{t}\left\|\dot{\alpha}_{\varepsilon}(s)\right\|_{H^{m}(\Omega)} \mathrm{d} s+\int_{0}^{t}\left\|\dot{e}_{\varepsilon}(s)\right\|_{2} \mathrm{~d} s+\int_{0}^{t}\left\|\dot{p}_{\varepsilon}(s)\right\|_{1} \mathrm{~d} s=: s_{\varepsilon}^{\circ}(t)
$$

is uniformly bounded in $\varepsilon$ and $t$. Following [25,7], we observe that $s_{\varepsilon}^{\circ}$ admits an inverse function $t_{\varepsilon}^{\circ}$ which is a contraction from a suitable interval $[0, S]$ into $[0, T]$ for every $\varepsilon$; moreover, the 5 -tuples

$$
\left(\alpha_{\varepsilon}, u_{\varepsilon}, e_{\varepsilon}, p_{\varepsilon}, \mathrm{Id}\right) \circ t_{\varepsilon}^{\circ}=:\left(\alpha_{\varepsilon}^{\circ}, u_{\varepsilon}^{\circ}, e_{\varepsilon}^{\circ}, p_{\varepsilon}^{\circ}, t_{\varepsilon}^{\circ}\right)
$$

are Lipschitz in time, uniformly with respect to $\varepsilon$. 
Rescaled quasistatic viscosity evolutions. Passing to the limit as $\varepsilon \rightarrow 0$, we obtain (up to subsequences) a 5 -tuple of Lipschitz functions $\left(\alpha^{\circ}, u^{\circ}, e^{\circ}, p^{\circ}, t^{\circ}\right)$ from $[0, S]$ into $H^{m}(\Omega ;[0,1]) \times B D(\Omega) \times$ $L^{2}\left(\Omega ; \mathbb{M}_{\text {sym }}^{n \times n}\right) \times M_{b}\left(\Omega \cup \partial_{D} \Omega ; \mathbb{M}_{D}^{n \times n}\right) \times[0, T]$, which we call rescaled quasistatic viscosity evolution (see Definition 4.1 and Theorem 4.4). The limit evolution inherits some properties of the viscous approximants; more precisely, $\alpha^{\circ}$ is nonincreasing and the following conditions hold true:

(ev1) kinematic condition and equilibrium: for every $s \in[0, S]$

$$
\left(u^{\circ}(s), e^{\circ}(s), p^{\circ}(s)\right) \in A\left(w^{\circ}(s)\right), \quad \operatorname{div} \sigma^{\circ}(s)=0 \text { in } \Omega, \quad\left[\sigma^{\circ}(s) \nu\right]=0 \text { on } \partial_{N} \Omega ;
$$

(ev2) stress constraint: for every $s \in[0, S]$ and a.e. $x \in \Omega$

$$
\sigma_{D}^{\circ}(s, x) \in K\left(\alpha^{\circ}(s, x)\right),
$$

where $\sigma^{\circ}(s):=\mathbb{C}\left(\alpha^{\circ}(s)\right) e^{\circ}(s)$ is the stress and $w^{\circ}(s):=w\left(t^{\circ}(s)\right)$ the rescaled prescribed boundary displacement.

The technique adopted in the passage to the limit as $\varepsilon \rightarrow 0$ leads us to consider $s=\left(t^{\circ}\right)^{-1}(t)$ as a "slow" time scale used to reparametrize the process. The discontinuities of the evolution in the original time scale $t=t^{\circ}(s)$ correspond now to the set

$$
U^{\circ}:=\left\{s \in[0, S]: t^{\circ} \text { is constant in a neighbourhood of } s\right\},
$$

where the rescaled evolution gives a continuous interpolation between the states before and after each jump. In the remaining part of the interval $[0, S]$ the limit evolution is quasistatic and fulfills the following first-order stability condition,

(ev3) Kuhn-Tucker inequality: for every $s \in[0, S] \backslash U^{\circ}$

$$
\left\langle\partial_{\alpha} \mathcal{E}_{\lambda}\left(\alpha^{\circ}(s), e^{\circ}(s) ; p^{\circ}, s\right), \beta\right\rangle \geq 0 \quad \text { for every } \beta \in H^{m}(\Omega), \beta \leq 0 .
$$

In contrast, in $U^{\circ}$ the limit evolution is governed by a variational inequality of viscous type, representing a fast unstable propagation in the original time scale.

Finally, the energy dissipated in the processes of damage and plasticity is determined by the following equality,

(ev4) energy balance: for every $s \in[0, S]$

$$
\begin{aligned}
& \mathcal{E}\left(\alpha^{\circ}(s), e^{\circ}(s)\right)+\lambda \int_{0}^{s} \mathcal{H}\left(\alpha^{\circ}(s), \dot{p}^{\circ}(\tau)\right) \mathrm{d} \tau+(1-\lambda) \int_{0}^{s} \mathcal{H}\left(\alpha^{\circ}(\tau), \dot{p}^{\circ}(\tau)\right) \mathrm{d} \tau \\
& +\int_{0}^{s}\left\|\dot{\alpha}^{\circ}(\tau)\right\|_{2} \Phi\left(\partial_{\alpha} \mathcal{E}_{\lambda}\left(\alpha^{\circ}(s), e^{\circ}(s) ; p^{\circ}, s\right)\right) \mathrm{d} \tau=\mathcal{E}\left(\alpha_{0}, e_{0}\right)+\int_{0}^{s}\left\langle\sigma^{\circ}(\tau), E \dot{w}^{\circ}(\tau)\right\rangle \mathrm{d} \tau,
\end{aligned}
$$

where $\Phi(f):=\sup \left\{\langle-f, \beta\rangle: \beta \in H^{m}(\Omega), \beta \leq 0\right.$, and $\left.\|\beta\|_{2} \leq 1\right\}$ for every $f \in\left(H^{m}(\Omega)\right)^{\prime}$. The fourth term in the left-hand side gives a null contribution in each subinterval of $[0, S] \backslash U^{\circ}$ and thus represents the energy dissipated during the unstable propagation in $U^{\circ}$, cf. (ev3); equivalently, it is also possible to formulate the energy balance in the original time scale, so this term concentrates at the time discontinuities. We also show in Lemma 3.4 that in general, for every $f \in\left(H^{m}(\Omega)\right)^{\prime}, \Phi(f)$ coincides with the $L^{2}$-distance of $f$ from the closed convex set $G$ of nonpositive elements of $\left(H^{m}(\Omega)\right)^{\prime}$, i.e., $\Phi(f)=\mathrm{d}_{2}(f, G):=\min \left\{\|g\|_{2}: g \in L^{2}(\Omega), f+g \in G\right\}$

The energy balance (ev4) also shows the role of the parameter $\lambda \in[0,1]$. In fact, notice that the damage variable acts differently in the second and in the third summand of the left-hand side, since it is computed at the final point of the interval in the former case, whilst it is variable in the latter. Therefore, if we derive in $s$ and take into account the cancellation, from the two dissipative integrals we obtain

$$
\mathcal{H}\left(\alpha^{\circ}(s), \dot{p}^{\circ}(s)\right)+\lambda\left\langle\partial_{\alpha} \mathcal{H}\left(\alpha^{\circ}(s), \dot{p}^{\circ}(s)\right), \dot{\alpha}^{\circ}(s)\right\rangle,
$$

the first term being the dissipation potential related to plasticity, the second one giving a contribution to the dissipation potential related to the damage variable: the latter is damped by the parameter $\lambda$. Tuning $\lambda$ between zero and one, we account for different effects of the plasticity on the damage process; indeed, the bigger is $\lambda$, the easier it is to damage a portion of the material affected by plastic strain's changes. Thus the parameter $\lambda$ is related to a fatigue phenomenon. Setting $\lambda=0$ leads to an energy balance analogous to the one of [7]; the choice $\lambda=1$ was instead prescribed in $[1,2]$.

We conclude our introduction by underlining that our rescaled quasistatic viscosity evolutions satisfy at every time instant a weak form of the classical Prandtl-Reuss flow rule (that corresponds to a principle of maximum dissipation). Together with conditions (ev1) and (ev2), this flow rule characterizes the perfect plasticity. We thus obtain that the triple $\left(u^{\circ}, e^{\circ}, p^{\circ}\right)$ is an evolution for the (weakly formulated) model of perfect plasticity, with time-dependent elastic tensor and constraint sets, as in [35]. The elastic tensor depends indeed on the damage variable, whose evolution is governed by the Kuhn-Tucker criterion. We also prove that the functions $\left(u^{\circ}, e^{\circ}, p^{\circ}\right)$ may jump in the original time scale only if the damage variable $\alpha^{\circ}$ jumps. 


\section{Mechanical assumptions And mathematical tools}

In this section we present the assumptions required on our model and we collect some mathematical tools needed in the sequel of the paper. Damage phenomena are modeled according to Frémond's approach [13], while plasticity is formulated as in [5]. The assumptions are essentially as in [4] with minor changes.

Notation. We denote by $\mathcal{L}^{n}$ the Lebesgue measure on $\mathbb{R}^{n}$ and by $\mathcal{H}^{n-1}$ the $(n-1)$-dimensional Hausdorff measure. Given a locally compact subset $B$ of $\mathbb{R}^{n}$ and a finite dimensional Hilbert space $X$, the space of bounded $X$-valued Radon measures on $B$ is denoted by $M_{b}(B ; X)$, the indication of $X$ being omitted when $X=\mathbb{R}$. On $M_{b}(B ; X)$ we define the norm $\|\mu\|_{1}:=|\mu|(B)$, where $|\mu| \in M_{b}(B)$ is the total variation of the measure $\mu$. By the Riesz Representation Theorem, $M_{b}(B ; X)$ can be identified with the dual of $C_{0}(B ; X)$, the space of continuous functions $\varphi: B \rightarrow X$ such that $\{|\varphi| \geq \varepsilon\}$ is compact for every $\varepsilon>0$ (see, e.g., [32, Theorem 6.19]). On $M_{b}(B ; X)$ we shall use the weak* topology, defined by duality. For $v \in L^{p}(B ; X), p \in[1,+\infty]$, the symbol $\|v\|_{p}$ denotes its $L^{p}$ norm. If $L^{1}(B ; X)$ is regarded as a subspace of $M_{b}(B ; X)$, then $\|\cdot\|_{1}$ coincides with the induced norm. The brackets $\langle\cdot, \cdot\rangle$ denote the product between dual spaces.

If $X_{1}, X_{2}$ are Banach spaces, $\operatorname{Lin}\left(X_{1} ; X_{2}\right)$ denotes the space of linear operators from $X_{1}$ into $X_{2}$, endowed with the usual operator norm. We will consider the space $\mathbb{M}_{\text {sym }}^{n \times n}$ of symmetric $n \times n$ matrices, endowed with the Euclidean scalar product $\xi: \eta:=\sum_{i j} \xi_{i j} \eta_{i j}$ and with the corresponding Euclidean norm $|\xi|:=(\xi: \xi)^{1 / 2}$, as well as the subspace $\mathbb{M}_{D}^{n \times n}$ of trace free matrices in $\mathbb{M}_{\text {sym }}^{n \times n}$. Given $\xi \in \mathbb{M}_{\text {sym }}^{n \times n}$, its orthogonal projection on $\mathbb{R} I$ is $\frac{1}{n} \operatorname{tr}(\xi) I$, while its orthogonal projection on $\mathbb{M}_{D}^{n \times n}$, called deviator, is $\xi_{D}:=\xi-\frac{1}{n}(\operatorname{tr} \xi) I$. Given two vectors $a=\left(a_{1}, \ldots, a_{n}\right), b=\left(b_{1}, \ldots, b_{n}\right) \in \mathbb{R}^{n}$, their symmetrized tensor product $a \odot b$ is the symmetric matrix whose entries are $\left(a_{i} b_{j}+a_{j} b_{i}\right) / 2,(i, j) \in\{1, \ldots, n\}^{2}$.

For $U$ open subset of $\mathbb{R}^{n}$ and $u \in L^{1}\left(U ; \mathbb{R}^{n}\right)$, the symbol $E u$ denotes the $\mathbb{M}_{s y m}^{n \times n}$-valued distribution on $U$ whose components are $E_{i j} u:=\frac{1}{2}\left(D_{j} u_{i}+D_{i} u_{j}\right),(i, j) \in\{1, \ldots, n\}^{2}$. The space $B D(U)$ of functions of bounded deformation, defined as the space of all $u \in L^{1}\left(U ; \mathbb{R}^{n}\right)$ with $E u \in M_{b}\left(U ; \mathbb{M}_{\text {sym }}^{n \times n}\right)$, is a Banach space with the norm $\|u\|_{1}+\|E u\|_{1}$. It turns out that $B D(U)$ is the dual of a normed linear space (see [23] and [37]); this allows us to consider the weak* topology on $B D(U)$. Equivalently, a sequence $\left\{u_{k}\right\}_{k}$ converges to $u$ weakly* in $B D(U)$ if and only if $u_{k} \rightarrow u$ strongly in $L^{1}\left(U ; \mathbb{R}^{n}\right)$ and $E u_{k} \rightarrow E u$ weakly* in $M_{b}\left(U ; \mathbb{M}_{s y m}^{n \times n}\right)$. If in addition $U$ is bounded and Lipschitz, for every function $u \in B D(U)$ it is possible to define the trace of $u$ on $\partial U$, still denoted by $u$, which belongs to $L^{1}\left(\partial U ; \mathbb{R}^{n}\right)$. If $u_{k}, u \in B D(U), u_{k} \rightarrow u$ strongly in $L^{1}\left(U ; \mathbb{R}^{n}\right)$, and $\left\|E u_{k}\right\|_{1} \rightarrow\|E u\|_{1}$, then $u_{k} \rightarrow u$ strongly in $L^{1}\left(\partial U ; \mathbb{R}^{n}\right)$ (see [36, Chapter II, Theorem 3.1]). Moreover, there exists a constant $C_{U}>0$, only depending on $U$, such that

$$
\|u\|_{1, U} \leq C_{U}\|u\|_{1, \partial U}+C_{U}\|E u\|_{1, U},
$$

$\|\cdot\|_{p, B}$ denoting the $L^{p}$ norm of a function on $B$ (see [36, Proposition 2.4 and Remark 2.5]). For the general properties of $B D(U)$ we refer to [36].

The body and its displacement. We consider an elastoplastic body whose reference configuration is a bounded, connected, open set $\Omega \subset \mathbb{R}^{n}, n \geq 2$, with Lipschitz boundary $\partial \Omega=\partial_{D} \Omega \cup \partial_{N} \Omega \cup N$. We assume that $\partial_{D} \Omega$ and $\partial_{N} \Omega$ are relatively open, $\partial_{D} \Omega \cap \partial_{N} \Omega=\emptyset, \mathcal{H}^{n-1}(N)=0$, and

$$
\partial_{D} \Omega \neq \emptyset \text {. }
$$

We assume that the common boundary $\Sigma:=\partial\left(\partial_{D} \Omega\right)=\partial\left(\partial_{N} \Omega\right)$ (topological notions refer here to the relative topology of $\partial \Omega$ ) satisfies the Kohn-Temam condition:

$\Sigma$ is a $(n-2)$-dimensional $C^{2}$ manifold,

$\partial \Omega$ is $C^{2}$ in a neighborhood of $\Sigma$.

The only role of this condition is to assure (1.20) below; another sufficient condition for (1.20) is for instance the one considered in [12, Theorem 6.6].

The displacement of the body is represented by a function $u \in B D(\Omega)$, so $E u$ is the corresponding linearized strain.

We study the evolution of the body under time-dependent external loading. Here we consider only Dirichlet boundary conditions on $\partial_{D} \Omega$ : such a choice notably simplifies the exposition. For including forces in a related model we refer to e.g. [7] and [34]. The prescribed boundary displacement is extended into the domain $\Omega$; at every time it is thus a function in $H^{1}\left(\mathbb{R}^{n} ; \mathbb{R}^{n}\right)$, whose trace on $\partial_{D} \Omega$ is the prescribed boundary value. For the time regularity of the boundary condition, see (1.27). 
The elastic and the plastic strain. Given a displacement $u \in B D(\Omega)$ and a boundary datum $w \in$ $H^{1}\left(\mathbb{R}^{n} ; \mathbb{R}^{n}\right)$, the elastic and the plastic strain, denoted by $e \in L^{2}\left(\Omega ; \mathbb{M}_{\text {sym }}^{n \times n}\right)$ and $p \in M_{b}\left(\Omega \cup \partial_{D} \Omega ; \mathbb{M}_{D}^{n \times n}\right)$, respectively, are assumed to satisfy the following weak kinematic compatibility conditions

$$
\begin{gathered}
E u=e+p \text { in } \Omega, \\
p=(w-u) \odot \nu \mathcal{H}^{n-1} \text { on } \partial_{D} \Omega .
\end{gathered}
$$

The set of admissible displacements and strains for a given boundary datum $w \in H^{1}\left(\mathbb{R}^{n} ; \mathbb{R}^{n}\right)$ on $\partial_{D} \Omega$ is

$$
A(w):=\left\{(u, e, p) \in B D(\Omega) \times L^{2}\left(\Omega ; \mathbb{M}_{s y m}^{n \times n}\right) \times M_{b}\left(\Omega \cup \partial_{D} \Omega ; \mathbb{M}_{D}^{n \times n}\right):(1.3) \text { holds }\right\} .
$$

The space of admissible plastic strains is defined by

$$
\begin{gathered}
\Pi(\Omega):=\left\{p \in M_{b}\left(\Omega \cup \partial_{D} \Omega ; \mathbb{M}_{D}^{n \times n}\right): \exists(u, w, e) \in B D(\Omega) \times H^{1}\left(\mathbb{R}^{n} ; \mathbb{R}^{n}\right) \times L^{2}\left(\Omega ; \mathbb{M}_{\text {sym }}^{n \times n}\right)\right. \\
\text { s.t. }(u, e, p) \in A(w)\} .
\end{gathered}
$$

The damage variable and the associated dissipation. Following Frémond's concept [13], the damage state of the body is represented by an internal variable $\alpha: \Omega \rightarrow[0,1]$, where $\alpha=1$ marks the sound material and $\alpha=0$ the most damaged state. For technical reasons, in our model the total energy (1.9) includes a regularizing term on the damage variable proportional to the seminorm

$$
|\alpha|_{m, 2}^{2}:=\sum_{|\beta|=m}\left\|D^{\beta} \alpha\right\|_{2}^{2}, \quad m:=\left[\frac{n}{2}\right]+1,
$$

where [.] denotes the integer part. The corresponding scalar product is

$$
\left\langle\alpha_{1}, \alpha_{2}\right\rangle_{m, 2}:=\sum_{|\beta|=m}\left\langle D^{\beta} \alpha_{1}, D^{\beta} \alpha_{2}\right\rangle_{2}
$$

We recall that $|\cdot|_{m, 2}$ is a seminorm on the space $H^{m}(\Omega)$ and that the norm

$$
\|\cdot\|_{m, 2}:=\|\cdot\|_{2}+|\cdot|_{m, 2}
$$

is equivalent to the usual norm in $H^{m}(\Omega)$ defined by $\|\alpha\|_{H^{m}(\Omega)}:=\sum_{|\beta| \leq m}\left\|D^{\beta} \alpha\right\|_{2}$. In particular, if a state has finite energy, the corresponding damage variable is in $H^{m}(\Omega)$, which is compactly embedded in $C(\bar{\Omega})$. Therefore, from now on we define all energy terms for $\alpha \in C(\bar{\Omega})$.

Given $\alpha_{0} \in C(\bar{\Omega})$, we denote the admissible damage states by

$$
\mathcal{D}\left(\alpha_{0}\right):=\left\{\alpha \in C(\bar{\Omega}): 0 \leq \alpha \leq \alpha_{0} \text { in } \bar{\Omega}\right\}
$$

so that

$$
\mathcal{D}\left(\alpha_{2}\right) \subset \mathcal{D}\left(\alpha_{1}\right) \text { for every } \alpha_{2} \in \mathcal{D}\left(\alpha_{1}\right) .
$$

Irreversibility is formulated in the following way: if $\alpha_{0}$ is the current damage state, then all future damage states are in $\mathcal{D}\left(\alpha_{0}\right)$. The total energy includes the energy dissipated by the body during the damage process. For $\alpha \in C(\bar{\Omega})$, the energy dissipated in damage growth is

$$
D(\alpha):=\int_{\Omega} d(\alpha(x)) \mathrm{d} x
$$

where

$$
\begin{gathered}
d \in C^{2}\left((0,+\infty) ; \mathbb{R}^{+}\right) \cap C\left([0,+\infty) ; \mathbb{R}^{+} \cup\{+\infty\}\right), \\
s^{2 n} d(s) \rightarrow+\infty \text { as } s \rightarrow 0^{+} .
\end{gathered}
$$

The latter requirements on $d$ force $\alpha$ to be positive; consequently, the material never reaches the most damaged state at any point. Since $d$ is a dissipated energy, it would be natural to assume it nonincreasing; however, this hypothesis is not needed to prove our results.

The stored elastic energy. For $(\alpha, e) \in C(\bar{\Omega} ;[0,1]) \times L^{2}\left(\Omega, \mathbb{M}_{\text {sym }}^{n \times n}\right)$, the stored elastic energy is

$$
\mathcal{Q}(\alpha, e):=\frac{1}{2} \int_{\Omega} \mathbb{C}(\alpha(x)) e(x): e(x) d x=\frac{1}{2}\langle\mathbb{C}(\alpha) e, e\rangle_{L^{2}\left(\Omega ; \mathbb{M}_{s y m}^{n \times n}\right)} .
$$

Following [5], [12], and [34], we assume that

$$
\mathbb{C}(\alpha) \xi:=\mathbb{C}_{D}(\alpha) \xi_{D}+\kappa(\alpha)(\operatorname{tr} \xi) I,
$$

where $\mathbb{C}_{D} \in L^{\infty}\left([0,1] ; \operatorname{Sym}\left(\mathbb{M}_{D}^{n \times n} ; \mathbb{M}_{D}^{n \times n}\right)\right), \kappa \in L^{\infty}([0,1])$, and

$$
\begin{gathered}
\mathbb{C}:[0,1] \rightarrow \operatorname{Lin}\left(\mathbb{M}_{\text {sym }}^{n \times n} ; \mathbb{M}_{\text {sym }}^{n \times n}\right) \text { is of class } C^{1,1}, \\
\alpha \mapsto \mathbb{C}(\alpha) \xi: \xi \text { is nondecreasing for every } \xi \in \mathbb{M}_{s y m}^{n \times n}, \\
\gamma_{1}|\xi|^{2} \leq \mathbb{C}(\alpha) \xi: \xi \leq \gamma_{2}|\xi|^{2} \quad \text { for every } \alpha \in[0,1], \xi \in \mathbb{M}_{\text {sym }}^{n \times n},
\end{gathered}
$$


where $\gamma_{1}, \gamma_{2}$ are positive constants independent of $\alpha$ and $\operatorname{Sym}\left(\mathbb{M}_{D}^{n \times n} ; \mathbb{M}_{D}^{n \times n}\right)$ is the set of symmetric endomorphisms on $\mathbb{M}_{D}^{n \times n}$. In particular, this implies

$$
|\mathbb{C}(\alpha) \xi| \leq 2 \gamma_{2}|\xi| .
$$

Assumption (1.7b) reflects the fact that the stiffness decreases as the material passes from the sound to the fully damaged state; at this last stage there is still elastic elastic response, by $(1.7 \mathrm{c})$, and thus the material is not completely damaged. Given $\alpha \in C(\bar{\Omega} ;[0,1])$, it is well known that the function $e \mapsto \mathcal{Q}(\alpha, e)$ is weakly lower semicontinuous on $L^{2}\left(\Omega ; \mathbb{M}_{\text {sym }}^{n \times n}\right)$.

In fact, (1.6) is not needed to prove existence results in plasticity, see e.g. [35]. Nevertheless, (1.6) is assumed for mechanical reasons, since purely volumetric deformations do not affect plastic behavior.

The total energy. Given a damage state $\alpha \in C(\bar{\Omega} ;[0,1])$ and an elastic strain $e \in L^{2}\left(\Omega, \mathbb{M}_{\text {sym }}^{n \times n}\right)$, the total energy of the configuration is

$$
\mathcal{E}(\alpha, e):= \begin{cases}\mathcal{Q}(\alpha, e)+D(\alpha)+\frac{\kappa}{2}|\alpha|_{m, 2}^{2} & \text { if } \alpha \in H^{m}(\Omega ;[0,1]), \\ +\infty & \text { otherwise. }\end{cases}
$$

The previous assumptions imply that $\mathcal{E}$ is lower semicontinuous with respect to the uniform convergence of the damage variable and the weak ${ }^{*}-L^{2}\left(\Omega ; \mathbb{M}_{s y m}^{n \times n}\right)$ convergence of the elastic strain. Moreover, for every $e \in L^{2}\left(\Omega ; \mathbb{M}_{s y m}^{n \times n}\right)$ the functional $H^{m}(\Omega ;[0,1]) \ni \alpha \mapsto \mathcal{E}(\alpha, e)$ is differentiable and

$$
\left\langle\partial_{\alpha} \mathcal{E}(\alpha, e), \beta\right\rangle=\frac{1}{2}\left\langle\mathbb{C}^{\prime}(\alpha) \beta e, e\right\rangle+\langle\partial D(\alpha), \beta\rangle+\kappa\langle\alpha, \beta\rangle_{m, 2}
$$

for every $\beta \in H^{m}(\Omega)$, where $\partial D(\alpha) \in M_{b}(\bar{\Omega})$ is the differential of $D$ at $\alpha$, given by $\langle\partial D(\alpha), \beta\rangle=$ $\int_{\Omega} d^{\prime}(\alpha(x)) \beta(x) \mathrm{d} x$.

The constraint sets and their support functions. The dissipation related to plasticity is defined through the so-called constraint sets, which are subsets of $\mathbb{M}_{D}^{n \times n}$ containing the admissible stresses. The coupling between damage and plasticity is reflected in the dependence of such sets on the damage variable. In a softening framework, we require the constraint sets $(K(\alpha))_{\alpha \in[0,1]}$ to fulfill the following conditions:

$$
\begin{gathered}
K(\alpha) \subset \mathbb{M}_{D}^{n \times n} \text { is closed and convex for every } \alpha \in[0,1], \\
B_{\widehat{r}}(0) \subset K\left(\alpha_{1}\right) \subset K\left(\alpha_{2}\right) \subset B_{\widehat{R}}(0) \text { for every } 0 \leq \alpha_{1} \leq \alpha_{2} \leq 1, \\
\mathrm{~d}_{\mathscr{H}}\left(K\left(\alpha_{1}\right), K\left(\alpha_{2}\right)\right) \leq C_{K}\left|\alpha_{1}-\alpha_{2}\right| \text { for every } \alpha_{1}, \alpha_{2} \in[0,1],
\end{gathered}
$$

where $C_{K}, \widehat{r}$, and $\widehat{R}$ are positive constants and $\mathrm{d}_{\mathscr{C}}$ is the Hausdorff distance, which is defined for two compact sets $K_{1}, K_{2}$ by

$$
\mathrm{d} \mathscr{H}\left(K_{1}, K_{2}\right):=\max \left\{\sup _{x \in K_{1}} \operatorname{dist}\left(x, K_{2}\right), \sup _{x \in K_{2}} \operatorname{dist}\left(x, K_{1}\right)\right\},
$$

with the conventions $\mathrm{d}_{\mathscr{H}}(x, \emptyset)=\operatorname{diam}(\Omega)$ and $\sup \emptyset=0$.

Let us introduce the support function $H:[0,1] \times \mathbb{M}_{D}^{n \times n} \rightarrow \mathbb{R}^{+}$defined by

$$
H(\alpha, \xi):=\sup _{\sigma \in K(\alpha)} \sigma: \xi \text { for every } \alpha \in[0,1] .
$$

The following Lemma shows that $H$ has the following properties:

$$
H \text { is continuous, }
$$

$$
0 \leq H\left(\alpha_{2}, \xi\right)-H\left(\alpha_{1}, \xi\right) \leq C_{K}\left(\alpha_{2}-\alpha_{1}\right) \text { for } 0 \leq \alpha_{1} \leq \alpha_{2} \leq 1 \text { and } \xi \in \mathbb{M}_{D}^{n \times n},|\xi|=1,
$$

$\xi \mapsto H(\alpha, \xi)$ is convex and positively one-homogeneous for every $\alpha \in[0,1]$,

$$
r|\xi| \leq H(\alpha, \xi) \leq R|\xi| \text { for every } \alpha \in[0,1] \text { and } \xi \in \mathbb{M}_{D}^{n \times n},
$$

with $r, R$ positive constants independent of $\alpha$ and $\xi$.

Lemma 1.1. Conditions (1.11) imply (1.12).

Proof. We first prove (1.12b). Let us fix $0 \leq \alpha_{1} \leq \alpha_{2} \leq 1, \xi \in \mathbb{M}_{D}^{n \times n}$ with $|\xi|=1$, and let $\left(E_{1}, \ldots, E_{N}\right)$, $N:=\frac{n(n+1)}{2}-1$, be an orthonormal basis of $\mathbb{M}_{D}^{n \times n}$ with $\xi=E_{1}$. Hence, for every $\alpha$

$$
H\left(\alpha, E_{1}\right)=\max _{\sigma \in K(\alpha)} \sigma^{1},
$$

where $\sigma^{i}$ is the $i$-th component of $\sigma$ in the choosen basis. Since the constraint sets are closed we have that $K\left(\alpha_{2}\right)$ is contained in the tubular neighbourhood $\bigcup_{\sigma \in K\left(\alpha_{1}\right)} \bar{B}\left(\sigma, \mathrm{d} \mathscr{H}\left(K\left(\alpha_{1}\right), K\left(\alpha_{2}\right)\right)\right)$ of $K\left(\alpha_{1}\right)$. Then for all $\sigma \in K\left(\alpha_{2}\right)$ we have $\sigma^{1} \leq H\left(\alpha_{1}, E_{1}\right)+\mathrm{d}_{\mathscr{H}}\left(K\left(\alpha_{1}\right), K\left(\alpha_{2}\right)\right)$; assuming the opposite would imply $\sigma \notin \bigcup_{\sigma \in K\left(\alpha_{1}\right)} \bar{B}\left(\sigma, \mathrm{d}_{\mathscr{H}}\left(K\left(\alpha_{1}\right), K\left(\alpha_{2}\right)\right)\right)$. Taking the supremum for $\sigma \in K\left(\alpha_{2}\right)$ we get

$$
H\left(\alpha_{2}, \xi\right)-H\left(\alpha_{1}, \xi\right) \leq \mathrm{d}_{\mathscr{H}}\left(K\left(\alpha_{1}\right), K\left(\alpha_{2}\right)\right) \text { for every }|\xi|=1,
$$


and together with (1.11b) and (1.11c) we get (1.12b). Properties (1.12c) and (1.12d) follow immediately from (1.11a) and (1.11b), while (1.12a) comes from (1.12b) and the fact that the functions $\xi \mapsto H(\alpha, \xi)$ are convex with respect to $\xi$ for every $\alpha$ and locally equi-bounded with respect to $\alpha$, by (1.12d).

For some of the results (case $\lambda \in(0,1]$ in the following Sections) we will make the additional assumptions that

$$
\begin{gathered}
\xi \mapsto H\left(\alpha_{2}, \xi\right)-H\left(\alpha_{1}, \xi\right) \text { is convex for every } 0 \leq \alpha_{1} \leq \alpha_{2} \leq 1, \\
\alpha \mapsto H(\alpha, \xi) \in C^{1,1}([0,1]) \text { and }\left|\partial_{\alpha} H\left(\alpha_{2}, \xi\right)-\partial_{\alpha} H\left(\alpha_{1}, \xi\right)\right| \leq \bar{C}_{K}\left|\alpha_{1}-\alpha_{2}\right| \text { for }|\xi|=1,
\end{gathered}
$$

with $\bar{C}_{K}$ uniform with respect to $\alpha$ and $\xi$.

All the previous assumptions are satisfied in the usual multiplicative example: $K(\alpha):=V(\alpha) K(1)$, for $V \in C^{1,1}([0,1] ;[\bar{m}, \bar{M}])$ nondecreasing, $0<\bar{m}<\bar{M}$, so $H(\alpha, \xi)=V(\alpha) H(1, \xi)$.

The plastic potential. The plastic potential $\mathcal{H}: C(\bar{\Omega} ;[0,1]) \times M_{b}\left(\Omega \cup \partial_{D} \Omega ; \mathbb{M}_{D}^{n \times n}\right) \rightarrow \mathbb{R}$ is defined by

$$
\mathcal{H}(\alpha, p):=\int_{\Omega \cup \partial_{D} \Omega} H\left(\alpha(x), \frac{\mathrm{d} p}{\mathrm{~d} \mu}(x)\right) \mathrm{d} \mu(x),
$$

where $\mu \in M_{b}\left(\Omega \cup \partial_{D} \Omega\right)^{+}$is a measure such that $p \ll \mu$ and $\frac{\mathrm{d} p}{\mathrm{~d} \mu}$ is the Radon-Nikodym derivative of $p$ with respect to $\mu$; since $H(\alpha(x), \cdot)$ is one-homogeneous, the definition is actually independent of $\mu$. We refer to [14] for the theory of convex functions of measures. By [3, Proposition 2.37]

$$
p \mapsto \mathcal{H}(\alpha, p) \text { is convex and positively one-homogeneous for every } \alpha \in C(\bar{\Omega} ;[0,1]) \text {. }
$$

In particular,

$$
\mathcal{H}\left(\alpha, p_{1}+p_{2}\right) \leq \mathcal{H}\left(\alpha, p_{1}\right)+\mathcal{H}\left(\alpha, p_{2}\right)
$$

for every $\alpha \in C(\bar{\Omega} ;[0,1])$ and $p_{1}, p_{2} \in M_{b}\left(\Omega \cup \partial_{D} \Omega ; \mathbb{M}_{D}^{n \times n}\right)$. Since $\left|\frac{\mathrm{d} p}{\mathrm{~d}|p|}(x)\right|=1$ for $|p|$-a.e. $x \in \Omega \cup \partial_{D} \Omega$, by (1.12) we have

$$
r\|p\|_{1} \leq \mathcal{H}(\alpha, p) \leq R\|p\|_{1}
$$

and

$$
0 \leq \mathcal{H}\left(\alpha_{2}, p\right)-\mathcal{H}\left(\alpha_{1}, p\right) \leq C_{K}\left\|\alpha_{1}-\alpha_{2}\right\|_{\infty}\|p\|_{1} \quad \text { for } 0 \leq \alpha_{1} \leq \alpha_{2} \leq 1
$$

Therefore, by Reshetnyak's Lower Semicontinuity Theorem, if $\alpha_{k}$ and $p_{k}$ are sequences in $C(\bar{\Omega} ;[0,1])$ and $M_{b}\left(\Omega \cup \partial_{D} \Omega ; \mathbb{M}_{D}^{n \times n}\right)$ such that $\alpha_{k} \rightarrow \alpha$ uniformly and $p_{k} \rightarrow p$ weakly* in $M_{b}\left(\Omega \cup \partial_{D} \Omega ; \mathbb{M}_{D}^{n \times n}\right)$, then

$$
\mathcal{H}(\alpha, p) \leq \liminf _{k \rightarrow \infty} \mathcal{H}\left(\alpha_{k}, p_{k}\right)
$$

Furthermore, under the additional hypothesis (1.13), the functional $C(\bar{\Omega} ;[0,1]) \ni \alpha \mapsto \mathcal{H}(\alpha, p)$ is differentiable, $\partial_{\alpha} H$ is convex in the second variable, and $\partial_{\alpha} \mathcal{H}(\alpha, p) \in M_{b}(\bar{\Omega})$ is given by

$$
\left\langle\partial_{\alpha} \mathcal{H}(\alpha, p), \beta\right\rangle=\int_{\Omega \cup \partial_{D} \Omega} \partial_{\alpha} H\left(\alpha(x), \frac{\mathrm{d} p}{\mathrm{~d}|p|}(x)\right) \beta(x) \mathrm{d}|p|(x) \quad \text { for every } \beta \in C(\bar{\Omega}) ;
$$

thus by $(1.13 \mathrm{~b})$

$$
\left\|\partial_{\alpha} \mathcal{H}(\alpha, p)\right\|_{1} \leq \bar{R}\|p\|_{1},
$$

for a suitable $\bar{R}$ depending only on $H$, and

$$
\left\|\partial_{\alpha} \mathcal{H}\left(\alpha_{1}, p\right)-\partial_{\alpha} \mathcal{H}\left(\alpha_{2}, p\right)\right\|_{1} \leq \bar{C}_{K}\left\|\alpha_{1}-\alpha_{2}\right\|_{\infty}\|p\|_{1}
$$

Stress-strain duality. We now recall the notion of stress-strain duality, basing on [19], [5], and the more recent extension to Lipschitz boundaries [12], to which we refer for the properties mentioned below. We define

$$
\Sigma(\Omega):=\left\{\sigma \in L^{2}\left(\Omega ; \mathbb{M}_{\text {sym }}^{n \times n}\right): \operatorname{div} \sigma \in L^{n}\left(\Omega ; \mathbb{R}^{n}\right), \sigma_{D} \in L^{\infty}\left(\Omega ; \mathbb{M}_{D}^{n \times n}\right)\right\}
$$

and, for $\sigma \in \Sigma(\Omega)$ and $p \in \Pi(\Omega)$,

$$
\left\langle\left[\sigma_{D}: p\right], \varphi\right\rangle:=-\int_{\Omega} \varphi \sigma \cdot(e-E w) \mathrm{d} x-\int_{\Omega} \sigma \cdot[(u-w) \odot \nabla \varphi] \mathrm{d} x-\int_{\Omega} \varphi(\operatorname{div} \sigma) \cdot(u-w) \mathrm{d} x
$$

for every $\varphi \in C_{c}^{\infty}\left(\mathbb{R}^{n}\right)$, where $u$ and $e$ are such that $(u, e, p) \in A(w)$. (The definition is indeed independent of $u$ and $e$.) Under the previous assumptions $\sigma \in L^{r}\left(\Omega ; \mathbb{M}_{\text {sym }}^{n \times n}\right)$ for every $r<\infty, u \in L^{\frac{n}{n-1}}\left(\Omega ; \mathbb{R}^{n}\right)$, and $\left[\sigma_{D}: p\right]$ is a bounded Radon measure such that $\left\|\left[\sigma_{D}: p\right]\right\|_{1} \leq\left\|\sigma_{D}\right\|_{\infty}\|p\|_{1}$ in $\mathbb{R}^{n}$. Using the restriction to $\Omega \cup \partial_{D} \Omega$, we also define

$$
\left\langle\sigma_{D} \mid p\right\rangle:=\left[\sigma_{D}: p\right]\left(\Omega \cup \partial_{D} \Omega\right) .
$$

For $\sigma \in L^{2}\left(\Omega ; \mathbb{M}_{s y m}^{n \times n}\right)$ and $\operatorname{div} \sigma \in L^{2}\left(\Omega ; \mathbb{R}^{n}\right)$, we denote by $[\sigma \nu]$ the normal trace on $\partial \Omega$, in general defined as a distribution. When $\sigma \in C\left(\Omega ; \mathbb{M}_{s y m}^{n \times n}\right)$ we have $[\sigma \nu]=\sigma \nu$ where the right-hand side is the 
pointwise product between the matrix $\sigma(x)$ and the normal vector $\nu(x)$ at each $x \in \partial \Omega$. By (1.19), if $[\sigma \nu] \in L^{\infty}\left(\partial_{N} \Omega ; \mathbb{R}^{n}\right)$ and (1.2) holds, we obtain the integration-by-parts formula

$\left\langle\sigma_{D} \mid p\right\rangle=-\langle\sigma, e-E w\rangle-\langle\operatorname{div} \sigma, u-w\rangle+\langle[\sigma \nu], u-w\rangle_{\partial_{N} \Omega} \quad$ for every $\sigma \in \Sigma(\Omega)$ and $(u, e, p) \in A(w)$.

For $\alpha \in C(\bar{\Omega})$ let

$$
\mathcal{K}_{\alpha}(\Omega):=\left\{\sigma \in L^{2}\left(\Omega ; \mathbb{M}_{\text {sym }}^{n \times n}\right): \operatorname{div} \sigma \in L^{n}\left(\Omega ; \mathbb{R}^{n}\right), \sigma_{D}(x) \in K(\alpha(x)) \text { for a.e. } x \in \Omega\right\} .
$$

Since the multifunction $\alpha \in[0,1] \mapsto K(\alpha)$ is continuous, from [12, Proposition 3.9] (which holds also if $\operatorname{div} \sigma$ is not identically 0$)$ it follows that for every $\sigma \in \mathcal{K}_{\alpha}(\Omega)$

$$
H\left(\alpha, \frac{\mathrm{d} p}{\mathrm{~d}|p|}\right)|p| \geq\left[\sigma_{D}: p\right] \quad \text { as measures on } \Omega \cup \partial_{D} \Omega,
$$

and, arguing as in [33, Theorem 3.6 and Corollary 3.8], we deduce that, for every $p \in \Pi(\Omega)$

$$
\mathcal{H}(\alpha, p)=\sup _{\sigma \in \mathcal{K}_{\alpha}(\Omega)}\left\langle\sigma_{D} \mid p\right\rangle .
$$

The plastic dissipation. We are now in a position to define the dissipation related to plasticity. A function $p:[0, T] \rightarrow M_{b}\left(\Omega \cup \partial_{D} \Omega ; \mathbb{M}_{D}^{n \times n}\right)$ will be regarded as a function defined on the time interval $[0, T]$ with values in the dual of the space $C_{0}\left(\Omega \cup \partial_{D} \Omega ; \mathbb{M}_{D}^{n \times n}\right)$. This space can be identified with the space of functions in $C\left(\bar{\Omega} ; \mathbb{M}_{D}^{n \times n}\right)$ vanishing on $\overline{\partial_{N} \Omega}$. For every $s, t \in[0, T]$ with $s \leq t$ the total variation of $p$ on $[s, t]$ is

$$
\mathcal{V}(p ; s, t):=\sup \left\{\sum_{j=1}^{N}\left\|p\left(t_{j}\right)-p\left(t_{j-1}\right)\right\|_{1}: s=t_{0}<t_{1}<\cdots<t_{N}=t, N \in \mathbb{N}\right\} .
$$

Let $\alpha:[0, T] \rightarrow C(\bar{\Omega} ;[0,1])$. The plastic dissipation in the time interval $[s, t]$ is defined by

$$
\mathcal{V}_{\mathcal{H}}(\alpha, p ; s, t):=\sup \left\{\sum_{j=1}^{N} \mathcal{H}\left(\alpha\left(t_{j}\right), p\left(t_{j}\right)-p\left(t_{j-1}\right)\right): s=t_{0}<t_{1}<\cdots<t_{N}=t, N \in \mathbb{N}\right\} .
$$

To ease the reading, when $\alpha \in C(\bar{\Omega} ;[0,1])$ does not depend on time we use the following notation:

$$
\widehat{\mathcal{V}}_{\mathcal{H}}(\alpha, p ; s, t):=\sup \left\{\sum_{j=1}^{N} \mathcal{H}\left(\alpha, p\left(t_{j}\right)-p\left(t_{j-1}\right)\right): s=t_{0}<t_{1}<\cdots<t_{N}=t, N \in \mathbb{N}\right\} .
$$

Assume now that $p \in A C\left([s, t] ; M_{b}\left(\Omega \cup \partial_{D} \Omega ; \mathbb{M}_{D}^{n \times n}\right)\right)$; then for a.e. $t \in[0, T]$ its weak* derivative (i.e., the weak* limit of the difference quotient) is well defined, see [5, Theorem 7.1], and we denote il by $\dot{p}(t)$. By [4, Lemma A.1], if $\alpha$ is strongly continuous (with respect to the strong topology of $C(\bar{\Omega})$ ) at a.e. $\tau \in[s, t]$, then

$$
\mathcal{V}_{\mathcal{H}}(\alpha, p ; s, t)=\int_{s}^{t} \mathcal{H}(\alpha(\tau), \dot{p}(\tau)) \mathrm{d} \tau .
$$

Notice that the condition on $\alpha$ is satisfied if $\alpha \in L^{\infty}\left(0, T ; H^{m}(\Omega)\right)$ and it is increasing in time, using [4, Lemma A.2] and the compact embedding of $H^{m}(\Omega)$ into $C(\bar{\Omega})$. Moreover, under the additional assumption (1.13), the functional $C(\bar{\Omega} ;[0,1]) \ni \alpha \mapsto \widehat{\mathcal{V}}_{\mathcal{H}}(\alpha, p ; s, t)$ is differentiable and

$$
\left\langle\partial_{\alpha} \widehat{\mathcal{V}}_{\mathcal{H}}(\alpha, p ; s, t), \beta\right\rangle=\int_{s}^{t}\left\langle\partial_{\alpha} \mathcal{H}(\alpha, \dot{p}(\tau)), \beta\right\rangle \mathrm{d} \tau
$$

for every $\beta \in C(\bar{\Omega})$. (See also (1.17) for the expression of $\partial_{\alpha} \mathcal{H}(\alpha, \dot{p}(\tau)$ ).)

The generalized energy. In this paper, we account for different possible couplings between damage and plasticity, which are described by introducing a parameter $\lambda$ that varies between zero and one. Accordingly, in the formulation of the problem we will often use the following functional,

$$
\mathcal{E}_{\lambda}(\alpha, e ; p, t):=\mathcal{E}(\alpha, e)+\lambda \widehat{\mathcal{V}}_{\mathcal{H}}(\alpha, p ; 0, t)
$$

where $\lambda \in[0,1]$.

We notice here that, assuming $p \in A C\left([0, t] ; M_{b}\left(\Omega \cup \partial_{D} \Omega ; \mathbb{M}_{D}^{n \times n}\right)\right)$ and (1.13), by (1.10) and (1.24) the functional $H^{m}(\Omega ;[0,1]) \ni \alpha \mapsto \mathcal{E}_{\lambda}(\alpha, e ; p, t)$ is differentiable and

$$
\left\langle\partial_{\alpha} \mathcal{E}_{\lambda}(\alpha, e ; p, t), \beta\right\rangle=\frac{1}{2}\left\langle\mathbb{C}^{\prime}(\alpha) \beta e, e\right\rangle+\langle\partial D(\alpha), \beta\rangle+\kappa\langle\alpha, \beta\rangle_{m, 2}+\lambda \int_{0}^{t}\left\langle\partial_{\alpha} \mathcal{H}(\alpha, \dot{p}(s)), \beta\right\rangle \mathrm{d} s
$$

for every $\beta \in H^{m}(\Omega)$. 
The external and initial data. For the time-dependent prescribed boundary conditions we require the following regularity:

$$
w \in H^{1}\left(0, T ; H^{1}\left(\mathbb{R}^{n} ; \mathbb{R}^{n}\right)\right) .
$$

We prescribe initial values $\alpha_{0}, u_{0}, e_{0}, p_{0}$ for damage, displacement, elastic, and plastic strain, respectively, that satisfy

$$
\alpha_{0} \in H^{2 m}(\Omega ;[c, 1]), e_{0} \in L^{4}(\Omega),\left(u_{0}, e_{0}, p_{0}\right) \in A(w(0)), \sigma_{0} \in \mathcal{K}_{\alpha_{0}}(\Omega), \operatorname{div} \sigma_{0}=0 \text { in } \Omega,
$$

with $c>0$ and $\sigma_{0}:=\mathbb{C}\left(\alpha_{0}\right) e_{0}$. Then the differential $\partial_{\alpha} \mathcal{E}\left(\alpha_{0}, e_{0}\right)$ given according to (1.10) by

$$
\left\langle\partial_{\alpha} \mathcal{E}\left(\alpha_{0}, e_{0}\right), \beta\right\rangle=\frac{1}{2}\left\langle\mathbb{C}^{\prime}\left(\alpha_{0}\right) \beta e_{0}, e_{0}\right\rangle_{2}+\left\langle\partial D\left(\alpha_{0}\right), \beta\right\rangle+\kappa\left\langle\alpha_{0}, \beta\right\rangle_{m, 2}
$$

for every $\beta \in C(\bar{\Omega})$, is represented by an $L^{2}$ function.

\section{Discrete-time Viscous apProximation}

To show the existence of quasistatic evolutions, we adopt the well-known method of vanishing viscosity, thus we study an approximate problem containing a viscous term driven by a (small) parameter $\varepsilon>0$. The existence of viscous approximations is proved by time-discretization through an incremental scheme. Therefore, we divide the time interval introducing $k$ equispaced nodes, solve a unilateral minimum problem (2.1) including the viscous dissipation, and take a piecewise affine interpolant of the resulting approximants; this is contained in the present section, together with some a-priori estimates on the approximants, which allow the passage to the limit as $k \rightarrow \infty$ and as $\varepsilon \rightarrow 0$, performed respectively in Section 3 and 4.

In particular, for the piecewise affine interpolants we show, using an argument developed in [34], that the time derivatives of the strains are bounded by the time derivatives of the damage and of the external loading, up to a multiplicative constant independent of $k$ and $\varepsilon$ (see Lemma 2.6). Combining this estimate with arguments similar to [18] allows us to prove that the approximate evolutions are $H^{1}$ in time uniformly with respect to $k$ for $\varepsilon$ fixed and that they are absolutely continuous in time, uniformly with respect to $\varepsilon$, too.

Henceforth, we always assume that (1.5), (1.7), (1.11) hold and that $w$ and $\left(\alpha_{0}, u_{0}, e_{0}, p_{0}\right)$ satisfy (1.27) and (1.28), respectively. For some of the results (case $\lambda \in(0,1])$ we will require also (1.13).

The incremental scheme. We set a sequence of subdivisions of the interval $[0, T]$ by fixing equispaced nodes $\left(t_{k}^{i}\right)_{0 \leq i \leq k}$,

$$
t_{k}^{i}:=\frac{i}{k} T \text {. }
$$

For every $k$, we set $\left(\alpha_{k}^{0},\left(u_{k}^{0}, e_{k}^{0}, p_{k}^{0}\right)\right):=\left(\alpha_{0},\left(u_{0}, e_{0}, p_{0}\right)\right)$ and for $i=1, \ldots, k$ we define $\left(\alpha_{k}^{i},\left(u_{k}^{i}, e_{k}^{i}, p_{k}^{i}\right)\right)$ as a solution to the incremental problem

$$
\min \left\{\mathcal{E}_{\lambda}\left(\beta, \eta ; p_{k}, t_{k}^{i-1}\right)+\mathcal{H}\left(\beta, q-p_{k}^{i-1}\right)+\frac{\varepsilon}{2 \tau}\left\|\beta-\alpha_{k}^{i-1}\right\|_{2}^{2}:(\beta,(u, \eta, q)) \in \mathcal{D}\left(\alpha_{k}^{i-1}\right) \times A\left(w_{k}^{i}\right)\right\},
$$

where $\tau=\tau_{k}:=\frac{1}{k}$ and we have used the following interpolants:

$$
\begin{gathered}
w_{k}^{i}:=w\left(t_{k}^{i}\right) \quad \text { for every } i=0, \ldots, k \\
p_{k}(t):=p_{k}^{j}+\frac{t-t_{k}^{j}}{\tau}\left(p_{k}^{j+1}-p_{k}^{j}\right) \quad \text { for } t \in\left[t_{k}^{j}, t_{k}^{j+1}\right) \text { and } j=0, \ldots, k-1 .
\end{gathered}
$$

We remark that, according to (1.25) and using (1.14) to evaluate the dissipation of a piecewise affine function,

$$
\mathcal{E}_{\lambda}\left(\beta, e ; p_{k}, t_{k}^{i}\right)=\mathcal{E}(\beta, e)+\lambda \sum_{j=1}^{i} \mathcal{H}\left(\beta, p_{k}^{j}-p_{k}^{j-1}\right) \quad \text { for } i=1, \ldots, k .
$$

The existence of solutions to problem (2.1) can be proved as in [4, Theorem 3.1] with straightforward modifications to account for the viscous term. In the following Lemma we collect some properties of discrete solutions which follow from [4, Lemmas 3.2 and 3.3], [5, Theorem 3.6], and [34, Lemma 3.2].

Lemma 2.1. If $\left(\alpha_{k}^{i},\left(u_{k}^{i}, e_{k}^{i}, p_{k}^{i}\right)\right)$ is a solution to problem (2.1), then the following equivalent conditions hold:

(a) $-\mathcal{H}\left(\alpha_{k}^{i}, p\right) \leq\left\langle\mathbb{C}\left(\alpha_{k}^{i}\right) e_{k}^{i}, e\right\rangle \leq \mathcal{H}\left(\alpha_{k}^{i},-p\right)$ for every $(u, e, p) \in A(0)$,

(b) $\mathbb{C}\left(\alpha_{k}^{i}\right) e_{k}^{i} \in \mathcal{K}_{\alpha_{k}^{i}}(\Omega), \quad \operatorname{div}\left(\mathbb{C}\left(\alpha_{k}^{i}\right) e_{k}^{i}\right)=0$ in $\Omega, \quad\left[\left(\mathbb{C}\left(\alpha_{k}^{i}\right) e_{k}^{i}\right) \nu\right]=0$ on $\partial_{N} \Omega$.

Moreover,

$$
\mathcal{E}_{\lambda}\left(\alpha_{k}^{i}, e_{k}^{i} ; p_{k}, t_{k}^{i-1}\right)+\frac{\varepsilon}{2 \tau}\left\|\alpha_{k}^{i}-\alpha_{k}^{i-1}\right\|_{2}^{2} \leq \mathcal{E}_{\lambda}\left(\beta, \eta ; p_{k}, t_{k}^{i-1}\right)+\mathcal{H}\left(\beta, q-p_{k}^{i}\right)+\frac{\varepsilon}{2 \tau}\left\|\beta-\alpha_{k}^{i-1}\right\|_{2}^{2}
$$

for every $(\beta,(u, \eta, q)) \in \mathcal{D}\left(\alpha_{k}^{i}\right) \times A\left(w_{k}^{i}\right)$, and

$$
\mathcal{Q}\left(\alpha_{k}^{i}, e_{k}^{i}\right)+\mathcal{Q}\left(\alpha_{k}^{i}, \eta-e_{k}^{i}\right) \leq \mathcal{Q}\left(\alpha_{k}^{i}, \eta\right)+\mathcal{H}\left(\alpha_{k}^{i}, q-p_{k}^{i}\right)
$$

for every $(u, \eta, q) \in A\left(w_{k}^{i}\right)$. 
Notice that we shall employ in the sequel only the latter of the equivalent conditions (a) and (b) above. We define the following piecewise constant and piecewise affine interpolants:

$$
\begin{aligned}
& \underline{\alpha}_{k}(t):=\alpha_{k}^{i}, \underline{u}_{k}(t):=u_{k}^{i}, \underline{e}_{k}(t):=e_{k}^{i}, \\
& \underline{p}_{k}(t):=p_{k}^{i}, \underline{\sigma}_{k}(t):=\mathbb{C}\left(\alpha_{k}^{i}\right) e_{k}^{i}, \underline{w}_{k}(t):=w_{k}^{i} \quad \text { for } t \in\left[t_{k}^{i}, t_{k}^{i+1}\right), \\
& \bar{\alpha}_{k}(t):=\alpha_{k}^{i}, \bar{u}_{k}(t):=u_{k}^{i}, \bar{e}_{k}(t):=e_{k}^{i}, \\
& \bar{p}_{k}(t):=p_{k}^{i}, \bar{\sigma}_{k}(t):=\mathbb{C}\left(\alpha_{k}^{i}\right) e_{k}^{i}, \bar{w}_{k}(t):=w_{k}^{i} \quad \text { for } t \in\left(t_{k}^{i-1}, t_{k}^{i}\right], \\
& \alpha_{k}(t):=\alpha_{k}^{i}+\frac{t-t_{k}^{i}}{\tau}\left(\alpha_{k}^{i+1}-\alpha_{k}^{i}\right), u_{k}(t):=u_{k}^{i}+\frac{t-t_{k}^{i}}{\tau}\left(u_{k}^{i+1}-u_{k}^{i}\right), e_{k}(t):=e_{k}^{i}+\frac{t-t_{k}^{i}}{\tau}\left(e_{k}^{i+1}-e_{k}^{i}\right), \\
& \sigma_{k}(t):=\mathbb{C}\left(\alpha_{k}(t)\right) e_{k}(t), w_{k}(t):=w_{k}^{i}+\frac{t-t_{k}^{i}}{\tau}\left(w_{k}^{i+1}-w_{k}^{i}\right) \quad \text { for } t \in\left[t_{k}^{i}, t_{k}^{i+1}\right) .
\end{aligned}
$$

(Recall also the definition of $p_{k}$ from (2.2).) Definitions (2.5a) and (2.5c) are given for $i=0 \ldots k-1$, and (2.5b) for $i=1 \ldots k$ instead. We define $\underline{\alpha}_{k}(T)=\alpha_{k}(T):=\alpha_{k}^{k}$ and $\bar{\alpha}_{k}(0):=\alpha_{0}$, and the same for the other interpolants. By definition $\underline{\alpha}_{k}, \bar{\alpha}_{k}$, and $\alpha_{k}$ are nonincreasing in time; moreover, $\left(\underline{u}_{k}(t), \underline{e}_{k}(t), \underline{p}_{k}(t)\right) \in$ $A\left(\underline{w}_{k}(t)\right),\left(\bar{u}_{k}(t), \bar{e}_{k}(t), \bar{p}_{k}(t)\right) \in A\left(\bar{w}_{k}(t)\right)$, and $\left(u_{k}(t), e_{k}(t), p_{k}(t)\right) \in A\left(w_{k}(t)\right)$ for every $t \in[0, T]$. We shall also use the notation

$$
\underline{\tau}_{k}(t):=t_{k}^{i} \text { if } t \in\left[t_{k}^{i}, t_{k}^{i+1}\right), \quad \bar{\tau}_{k}(t):=t_{k}^{i+1} \text { if } t \in\left(t_{k}^{i}, t_{k}^{i+1}\right] .
$$

The discrete energy inequality. We now derive an energy estimate for the solutions of the incremental problems. Let us fix $i \in\{1, \ldots, k\}$ and for a given integer $h$ with $1 \leq h \leq i$ let $u:=u_{k}^{h-1}-w_{k}^{h-1}+w_{k}^{h}$ and $\eta:=e_{k}^{h-1}-E w_{k}^{h-1}+E w_{k}^{h}$. Since $\left(\alpha_{k}^{h-1},\left(u, \eta, p_{k}^{h-1}\right)\right) \in \mathcal{D}\left(\alpha_{k}^{h-1}\right) \times A\left(w_{k}^{h}\right)$, by the minimality condition (2.1) we obtain

$$
\begin{aligned}
& \mathcal{E}_{\lambda}\left(\alpha_{k}^{h}, e_{k}^{h} ; p_{k}, t_{k}^{h-1}\right)+\mathcal{H}\left(\alpha_{k}^{h}, p_{k}^{h}-p_{k}^{h-1}\right)+\frac{\varepsilon}{2 \tau}\left\|\alpha_{k}^{h}-\alpha_{k}^{h-1}\right\|_{2}^{2} \\
& \quad \leq \mathcal{E}_{\lambda}\left(\alpha_{k}^{h-1}, e_{k}^{h-1} ; p_{k}, t_{k}^{h-1}\right)+\left\langle\sigma_{k}^{h-1}, E w_{k}^{h}-E w_{k}^{h-1}\right\rangle+\mathcal{Q}\left(\alpha_{k}^{h-1}, E w_{k}^{h}-E w_{k}^{h-1}\right),
\end{aligned}
$$

where we have used the identity

$$
\mathcal{Q}\left(\alpha, e_{1}+e_{2}\right)=\mathcal{Q}\left(\alpha, e_{1}\right)+\left\langle\mathbb{C}(\alpha) e_{1}, e_{2}\right\rangle+\mathcal{Q}\left(\alpha, e_{2}\right),
$$

which holds for every $\alpha \in H^{m}(\Omega ;[0,1])$ and $e_{1}, e_{2} \in L^{2}\left(\Omega ; \mathbb{M}_{\text {sym }}^{n \times n}\right)$. From the absolute continuity of $w$ with respect to $t$ we obtain

$$
w_{k}^{h}-w_{k}^{h-1}=\int_{t_{k}^{h-1}}^{t_{k}^{h}} \dot{w}(t) \mathrm{d} t,
$$

using the notion of Bochner integral for functions with values in $H^{1}\left(\mathbb{R}^{n} ; \mathbb{R}^{n}\right)$. This implies that

$$
E w_{k}^{h}-E w_{k}^{h-1}=\int_{t_{k}^{h-1}}^{t_{k}^{h}} E \dot{w}(t) \mathrm{d} t,
$$

where the integral is again in the sense of Bochner and the target space is $L^{2}\left(\mathbb{R}^{n} ; \mathbb{M}_{\text {sym }}^{n \times n}\right)$. By the continuity of $\mathcal{Q}$ and $(2.7)$ we get

$$
\mathcal{Q}\left(\alpha_{k}^{h-1}, E w_{k}^{h}-E w_{k}^{h-1}\right) \leq \gamma_{2}\left(\int_{t_{k}^{h-1}}^{t_{k}^{h}}\|E \dot{w}(t)\|_{2} \mathrm{~d} t\right)^{2} .
$$

Since

$$
\lambda \widehat{\mathcal{V}}_{\mathcal{H}}\left(\alpha_{k}^{h}, p_{k}, 0, t_{k}^{h-1}\right)+\mathcal{H}\left(\alpha_{k}^{h}, p_{k}^{h}-p_{k}^{h-1}\right)=\lambda \widehat{\mathcal{V}}_{\mathcal{H}}\left(\alpha_{k}^{h}, p_{k}, 0, t_{k}^{h}\right)+(1-\lambda) \mathcal{H}\left(\alpha_{k}^{h}, p_{k}^{h}-p_{k}^{h-1}\right),
$$

from $(2.3),(2.6)$, and (2.7) it follows that

$$
\begin{aligned}
& \mathcal{E}_{\lambda}\left(\alpha_{k}^{h}, e_{k}^{h} ; p_{k}, t_{k}^{h}\right)+(1-\lambda) \mathcal{H}\left(\alpha_{k}^{h}, p_{k}^{h}-p_{k}^{h-1}\right)+\frac{\varepsilon}{2 \tau}\left\|\alpha_{k}^{h}-\alpha_{k}^{h-1}\right\|_{2}^{2} \\
& \quad \leq \mathcal{E}_{\lambda}\left(\alpha_{k}^{h-1}, e_{k}^{h-1} ; p_{k}, t_{k}^{h-1}\right)+\int_{t_{k}^{h-1}}^{t_{k}^{h}}\left\langle\sigma_{k}^{h-1}, E \dot{w}(t)\right\rangle \mathrm{d} t+\omega_{k} \int_{t_{k}^{h-1}}^{t_{k}^{h}}\|E \dot{w}(t)\|_{2} \mathrm{~d} t,
\end{aligned}
$$

where

$$
\omega_{k}:=\gamma_{2} \max _{1 \leq h \leq k} \int_{t_{k}^{h-1}}^{t_{k}^{h}}\|E \dot{w}(t)\|_{2} \mathrm{~d} t \rightarrow 0
$$

by the absolute continuity of the integral. Iterating now the latter inequality for $1 \leq h \leq i$ amounts to the following property. 
Proposition 2.2. For every $i=1, \ldots, k$

$$
\begin{aligned}
& \mathcal{E}_{\lambda}\left(\alpha_{k}^{i}, e_{k}^{i} ; p_{k}, t_{k}^{i}\right)+(1-\lambda) \sum_{h=1}^{i} \mathcal{H}\left(\alpha_{k}^{h}, p_{k}^{h}-p_{k}^{h-1}\right)+\sum_{h=1}^{i} \frac{\varepsilon}{2 \tau}\left\|\alpha_{k}^{h}-\alpha_{k}^{h-1}\right\|_{2}^{2} \\
& \quad \leq \mathcal{E}\left(\alpha_{0}, e_{0}\right)+\int_{0}^{t_{k}^{i}}\left\langle\underline{\sigma}_{k}(s), E \dot{w}(s)\right\rangle \mathrm{d} s+\delta_{k},
\end{aligned}
$$

where $\delta_{k}:=\omega_{k} \int_{0}^{T}\|E \dot{w}(t)\|_{2} \mathrm{~d} t \rightarrow 0$.

A priori estimates. Using (1.7c) and (1.12d) in the left-hand side of (2.9), as well as (1.8) and the fact that the function $t \mapsto\|E \dot{w}(t)\|_{2}$ is integrable on $[0, T]$ in the right-hand side, we find that for every $k \in \mathbb{N}$ and $t \in\left(t_{k}^{i-1}, t_{k}^{i}\right]$

$$
\begin{aligned}
& \gamma_{1}\left\|\underline{e}_{k}(t)\right\|_{2}^{2}+D\left(\underline{\alpha}_{k}(t)\right)+\frac{\kappa}{2}\left|\underline{\alpha}_{k}(t)\right|_{m, 2}^{2}+r(1-\lambda) \sum_{h=1}^{i}\left\|p_{k}^{h}-p_{k}^{h-1}\right\|_{1}+\frac{\varepsilon}{2} \int_{0}^{t_{k}^{i}}\left\|\dot{\alpha}_{k}(s)\right\|_{2}^{2} \mathrm{~d} s \\
& \leq \mathcal{E}\left(\alpha_{0}, e_{0}\right)+2 \gamma_{2} \sup _{t \in[0, T]}\left\|\underline{e}_{k}(t)\right\|_{2} \int_{0}^{T}\|E \dot{w}(s)\|_{2} \mathrm{~d} s+\delta_{k} .
\end{aligned}
$$

Thus, by the Cauchy inequality,

$$
\sup _{t \in[0, T]}\left\|\underline{e}_{k}(t)\right\|_{2} \leq C .
$$

Henceforth, $C$ denotes a suitable constant depending only on $\gamma_{1}, \gamma_{2}, r$, and on the functions $\alpha_{0}, e_{0}$, and $w$. We immediately deduce that

$$
\begin{array}{r}
\sup _{t \in[0, T]} D\left(\underline{\alpha}_{k}(t)\right) \leq C, \\
\sup _{t \in[0, T]}\left\|\underline{\alpha}_{k}(t)\right\|_{m, 2} \leq C, \\
\varepsilon \int_{0}^{T}\left\|\dot{\alpha}_{k}(s)\right\|_{2}^{2} \mathrm{~d} s \leq C,
\end{array}
$$

and, from the definitions of the interpolants, that

$$
\mathcal{V}\left(\underline{p}_{k} ; 0, T\right)=\mathcal{V}\left(\bar{p}_{k} ; 0, T\right)=\mathcal{V}\left(p_{k} ; 0, T\right)=\sum_{i=1}^{k}\left\|p_{k}^{i}-p_{k}^{i-1}\right\|_{1} \leq C .
$$

Notice that analogous estimates to $(2.10),(2.11 \mathrm{a}),(2.11 \mathrm{~b})$ also hold for the other interpolants from $(2.5 \mathrm{~b})$ and $(2.5 \mathrm{c})$.

Next we show a bound from below on the damage variable, thanks to assumption (1.5).

Lemma 2.3. There exists $m_{0}>0$ independent of $\varepsilon, k, t$, such that

$$
\underline{\alpha}_{k}(t) \geq m_{0}, \quad \bar{\alpha}_{k}(t) \geq m_{0}, \quad \alpha_{k}(t) \geq m_{0} \quad \text { in } \bar{\Omega}
$$

for every $k \in \mathbb{N}$ and $t \in[0, T]$.

Proof. By (2.11b) and the continuous immersion $H^{m}(\Omega) \subset C^{0,1 / 2}(\Omega)$, cf. (1.4), there exists $\widetilde{C}$ independent of $\varepsilon, k, t$, with

$$
\left|\underline{\alpha}_{k}(t, x)-\underline{\alpha}_{k}(t, y)\right| \leq \widetilde{C}|x-y|^{1 / 2} \quad \text { for every } x, y \in \Omega .
$$

Let $M>0$; by (1.5b), there exists $\bar{\delta}>0$ such that $d(\delta)>M \delta^{-2 n}$ for every $0<\delta \leq \bar{\delta}$. Assume now that (2.13) does not hold, so we can find $k \in \mathbb{N}, t \in[0, T]$, and $\bar{x} \in \Omega$ such that $\underline{\alpha}_{k}(t, \bar{x})<\frac{\bar{\delta}}{2}$. Then $\underline{\alpha}_{k}(t, x)<\bar{\delta}$ for every $x \in B\left(\bar{x},(\bar{\delta} /(2 \widetilde{C}))^{2}\right)$. Therefore, $D\left(\underline{\alpha}_{k}(t)\right)>M \omega_{n} /(2 \widetilde{C})^{2 n}$, where $\omega_{n}$ is the measure of the unit ball in $\mathbb{R}^{n}$. Since $M$ is arbitrary, this contradicts (2.11a) and proves the thesis for $\underline{\alpha}_{k}$. The other statements are analogous.

By minimality, we get some differential conditions on the damage variable, which correspond to a discrete approximation of the Kuhn-Tucker conditions appearing in the following sections (cf. Definitions 3.1 and 4.1, and Propositions 3.2 and 4.3). We recall that we assume (1.13) when $\lambda \neq 0$; in that case we obtain the Kuhn-Tucker conditions (2.15). If $\lambda=0$, we would still be able to deduce (2.15) assuming (1.13); however, without that hypothesis, we can obtain the weaker version (2.14), which is sufficient for the subsequent applications of the lemma.

Lemma 2.4. Let $\varepsilon>0, k \in \mathbb{N}$, and $t \in(0, T) \backslash\left\{t_{k}^{1}, \ldots, t_{k}^{k-1}\right\}$. 
Case $\lambda=0$. We have

$$
\left\langle\partial_{\alpha} \mathcal{E}\left(\bar{\alpha}_{k}(t), \bar{e}_{k}(t)\right), \beta\right\rangle+\varepsilon\left\langle\dot{\alpha}_{k}(t), \beta\right\rangle_{2} \geq 0
$$

for every $\beta \in H_{-}^{m}(\Omega):=\left\{\beta \in H^{m}(\Omega): \beta \leq 0\right.$ in $\left.\Omega\right\}$, and

$$
\left\langle\partial_{\alpha} \mathcal{E}\left(\bar{\alpha}_{k}(t), \bar{e}_{k}(t)\right), \dot{\alpha}_{k}(t)\right\rangle+\varepsilon\left\|\dot{\alpha}_{k}(t)\right\|_{2}^{2} \leq C_{K} \tau\left\|\dot{\alpha}_{k}(t)\right\|_{\infty}\left\|\dot{p}_{k}(t)\right\|_{1}
$$

with $C_{K}$ introduced in $(1.11 \mathrm{c})$.

Case $\lambda \in(0,1]$. Under the additional assumption (1.13) we have

$$
\left\langle\partial_{\alpha} \mathcal{E}_{\lambda}\left(\bar{\alpha}_{k}(t), \bar{e}_{k}(t) ; p_{k}, \underline{\tau}_{k}(t)\right), \beta\right\rangle+\tau\left\langle\partial_{\alpha} \mathcal{H}\left(\bar{\alpha}_{k}(t), \dot{p}_{k}(t)\right), \beta\right\rangle+\varepsilon\left\langle\dot{\alpha}_{k}(t), \beta\right\rangle_{2} \geq 0
$$

for every $\beta \in H_{-}^{m}(\Omega)$, and

$$
\left\langle\partial_{\alpha} \mathcal{E}_{\lambda}\left(\bar{\alpha}_{k}(t), \bar{e}_{k}(t) ; p_{k}, \underline{\tau}_{k}(t)\right), \dot{\alpha}_{k}(t)\right\rangle+\varepsilon\left\|\dot{\alpha}_{k}(t)\right\|_{2}^{2}=-\tau\left\langle\partial_{\alpha} \mathcal{H}\left(\bar{\alpha}_{k}(t), \dot{p}_{k}(t)\right), \dot{\alpha}_{k}(t)\right\rangle .
$$

Proof. Let us denote $\dot{\alpha}_{k}^{i}:=\frac{\alpha_{k}^{i}-\alpha_{k}^{i-1}}{\tau}$. By (2.13), for every $\beta \in H_{-}^{m}(\Omega)$ there exists $\bar{\delta} \in(0,1)$ such that $\alpha_{k}^{i}+\delta \beta>0$ in $\bar{\Omega}$ for every $k, i$, and $0<\delta \leq \bar{\delta}$, which implies $\alpha_{k}^{i}+\delta \beta \in \mathcal{D}\left(\alpha_{k}^{i-1}\right)$. By minimality of $\alpha_{k}^{i}$

$$
\begin{aligned}
0 \leq & \mathcal{E}_{\lambda}\left(\alpha_{k}^{i}+\delta \beta, e_{k}^{i} ; p_{k}, t_{k}^{i-1}\right)+\mathcal{H}\left(\alpha_{k}^{i}+\delta \beta, p_{k}^{i}-p_{k}^{i-1}\right)+\frac{\varepsilon}{2 \tau}\left\|\alpha_{k}^{i}+\delta \beta-\alpha_{k}^{i-1}\right\|_{2}^{2} \\
& -\left(\mathcal{E}_{\lambda}\left(\alpha_{k}^{i}, e_{k}^{i} ; p_{k}, t_{k}^{i-1}\right)+\mathcal{H}\left(\alpha_{k}^{i}, p_{k}^{i}-p_{k}^{i-1}\right)+\frac{\varepsilon}{2 \tau}\left\|\alpha_{k}^{i}-\alpha_{k}^{i-1}\right\|_{2}^{2}\right) .
\end{aligned}
$$

If $\lambda=0$, dividing by $\delta$ and letting $\delta$ tend to 0 , we get $(2.14 \mathrm{a})$, since $\mathcal{H}\left(\alpha_{k}^{i}+\delta \beta, p_{k}^{i}-p_{k}^{i-1}\right) \leq \mathcal{H}\left(\alpha_{k}^{i}, p_{k}^{i}-p_{k}^{i-1}\right)$ by (1.16) (recall also the regularity assumptions on $\mathbb{C}$ and $D$ ). If $\lambda>0$, exploiting (1.13) and its consequences (1.17) and (1.18), we deduce (2.15a) using also (1.12c).

Moreover, $\alpha_{k}^{i}-\delta \dot{\alpha}_{k}^{i} \in \mathcal{D}\left(\alpha_{k}^{i-1}\right)$ for $\delta<\tau$, so

$$
\begin{aligned}
0 \leq & \mathcal{E}_{\lambda}\left(\alpha_{k}^{i}-\delta \dot{\alpha}_{k}^{i}, e_{k}^{i} ; p_{k}, t_{k}^{i-1}\right)+\mathcal{H}\left(\alpha_{k}^{i}-\delta \dot{\alpha}_{k}^{i}, p_{k}^{i}-p_{k}^{i-1}\right)+\frac{\varepsilon}{2 \tau}\left\|\alpha_{k}^{i}-\alpha_{k}^{i-1}+\delta \dot{\alpha}_{k}^{i}\right\|_{2}^{2} \\
& -\left(\mathcal{E}_{\lambda}\left(\alpha_{k}^{i}, e_{k}^{i} ; p_{k}, t_{k}^{i-1}\right)+\mathcal{H}\left(\alpha_{k}^{i}, p_{k}^{i}-p_{k}^{i-1}\right)+\frac{\varepsilon}{2 \tau}\left\|\alpha_{k}^{i}-\alpha_{k}^{i-1}\right\|_{2}^{2}\right) .
\end{aligned}
$$

If $\lambda=0$ we get

$$
\left\langle\partial_{\alpha} \mathcal{E}\left(\bar{\alpha}_{k}(t), \bar{e}_{k}(t)\right), \dot{\alpha}_{k}(t)\right\rangle+\varepsilon\left\|\dot{\alpha}_{k}(t)\right\|_{2}^{2}-\tau\left\langle\underline{\partial}_{\alpha}^{+} \mathcal{H}\left(\bar{\alpha}_{k}(t), \dot{p}_{k}(t)\right),-\dot{\alpha}_{k}(t)\right\rangle \leq 0,
$$

where

$$
\left\langle\underline{\partial}_{\alpha}^{+} \mathcal{H}\left(\bar{\alpha}_{k}(t), \dot{p}_{k}(t)\right),-\dot{\alpha}_{k}(t)\right\rangle:=\liminf _{\delta \rightarrow 0^{+}} \frac{\mathcal{H}\left(\bar{\alpha}_{k}(t)-\delta \dot{\alpha}_{k}(t), \dot{p}_{k}(t)\right)-\mathcal{H}\left(\bar{\alpha}_{k}(t), \dot{p}_{k}(t)\right)}{\delta},
$$

and then $(2.14 \mathrm{~b})$ follows by (1.16). If $\lambda \in(0,1]$, since we have already proved (2.15a) we get (2.15b) using again (1.13).

The next remark will turn out to be useful in the sequel.

Remark 2.5. Differentiating (2.8) with respect to the damage variable, we get that for every $\lambda \in(0,1]$ and $\beta \in H^{m}(\Omega)$

$$
\begin{aligned}
& \lambda\left\langle\partial_{\alpha} \widehat{\mathcal{V}}_{\mathcal{H}}\left(\bar{\alpha}_{k}(t), p_{k} ; 0, \underline{\tau}_{k}(t)\right), \beta\right\rangle+\tau\left\langle\partial_{\alpha} \mathcal{H}\left(\bar{\alpha}_{k}(t), \dot{p}_{k}(t)\right), \beta\right\rangle \\
& \quad=\lambda\left\langle\partial_{\alpha} \widehat{\mathcal{V}}_{\mathcal{H}}\left(\bar{\alpha}_{k}(t), p_{k} ; 0, \bar{\tau}_{k}(t)\right), \beta\right\rangle+(1-\lambda) \tau\left\langle\partial_{\alpha} \mathcal{H}\left(\bar{\alpha}_{k}(t), \dot{p}_{k}(t)\right), \beta\right\rangle,
\end{aligned}
$$

and then

$$
\begin{aligned}
& \left\langle\partial_{\alpha} \mathcal{E}_{\lambda}\left(\bar{\alpha}_{k}(t), \bar{e}_{k}(t) ; p_{k}, \underline{\tau}_{k}(t)\right), \beta\right\rangle+\tau\left\langle\partial_{\alpha} \mathcal{H}\left(\bar{\alpha}_{k}(t), \dot{p}_{k}(t)\right), \beta\right\rangle \\
& \quad=\left\langle\partial_{\alpha} \mathcal{E}_{\lambda}\left(\bar{\alpha}_{k}(t), \bar{e}_{k}(t) ; p_{k}, \bar{\tau}_{k}(t)\right), \beta\right\rangle+(1-\lambda) \tau\left\langle\partial_{\alpha} \mathcal{H}\left(\bar{\alpha}_{k}(t), \dot{p}_{k}(t)\right), \beta\right\rangle .
\end{aligned}
$$

The following lemma permits to bound the norm of $\dot{u}_{k}, \dot{e}_{k}$, and $\dot{p}_{k}$ by the norm of $\dot{\alpha}_{k}^{i}$ and $\dot{w}_{k}^{i}$ times a constant independent of $k$ and $\varepsilon$; this will be very useful to get the estimates in Propositions 2.7 and 2.8. In the proof we adapt an argument developed in [34, Lemma 3.3].

Lemma 2.6. For every $k \in \mathbb{N}$ and $0 \leq i \leq k-1$ let

$$
\widetilde{\omega}_{k}^{i}:=\left\|\alpha_{k}^{i+1}-\alpha_{k}^{i}\right\|_{\infty}+\left\|E w_{k}^{i+1}-E w_{k}^{i}\right\|_{2} .
$$

Then there exists a positive constant $C$ independent of $\varepsilon, k$, and $i$ such that

$$
\begin{aligned}
\left\|e_{k}^{i+1}-e_{k}^{i}\right\|_{2} & \leq C \widetilde{\omega}_{k}^{i}, \\
\left\|p_{k}^{i+1}-p_{k}^{i}\right\|_{1} & \leq C \widetilde{\omega}_{k}^{i}, \\
\left\|E u_{k}^{i+1}-E u_{k}^{i}\right\|_{1} & \leq C \widetilde{\omega}_{k}^{i}, \\
\left\|u_{k}^{i+1}-u_{k}^{i}\right\|_{B D} & \leq C\left(\widetilde{\omega}_{k}^{i}+\left\|w_{k}^{i+1}-w_{k}^{i}\right\|_{2}\right) .
\end{aligned}
$$

In particular, dividing by $\tau$, we have that for every $t \in(0, T) \backslash\left\{t_{k}^{1}, \ldots, t_{k}^{k-1}\right\}$

$$
\left\|\dot{e}_{k}(t)\right\|_{2}+\left\|\dot{p}_{k}(t)\right\|_{1}+\left\|E \dot{u}_{k}(t)\right\|_{1} \leq 3 C\left(\left\|\dot{\alpha}_{k}(t)\right\|_{\infty}+\left\|E \dot{w}_{k}(t)\right\|_{2}\right) .
$$


Finally, for every $t \in(0, T) \backslash\left\{t_{k}^{1}, \ldots, t_{k}^{k-1}\right\}$

$$
\mathcal{H}\left(\bar{\alpha}_{k}(t), \dot{p}_{k}(t)\right) \leq\left\langle\left(\bar{\sigma}_{k}(t)\right)_{D} \mid \dot{p}_{k}(t)\right\rangle+C \tau\left(\left\|\dot{\alpha}_{k}(t)\right\|_{\infty}^{2}+\left\|E \dot{w}_{k}(t)\right\|_{2}^{2}\right) .
$$

Proof. By Lemma 2.1 we obtain $\sigma_{k}^{i}:=\mathbb{C}\left(\alpha_{k}^{i}\right) e_{k}^{i} \in \mathcal{K}_{\alpha_{k}^{i}}(\Omega)$, div $\sigma_{k}^{i}=0$ in $\Omega$, and $\left[\sigma_{k}^{i} \nu\right]=0$ on $\partial_{N} \Omega$ for every $k$ and $i$. One can easily see that

$$
\left(\alpha_{k}^{i+1},\left(u_{k}^{i}+w_{k}^{i+1}-w_{k}^{i}, e_{k}^{i}+E\left(w_{k}^{i+1}-w_{k}^{i}\right), p_{k}^{i}\right)\right) \in \mathcal{D}\left(\alpha_{k}^{i}\right) \times A\left(w_{k}^{i+1}\right),
$$

and then by minimality

$$
\mathcal{Q}\left(\alpha_{k}^{i+1}, e_{k}^{i+1}\right)+\mathcal{H}\left(\alpha_{k}^{i+1}, p_{k}^{i+1}-p_{k}^{i}\right) \leq \mathcal{Q}\left(\alpha_{k}^{i+1}, e_{k}^{i}+E\left(w_{k}^{i+1}-w_{k}^{i}\right)\right) .
$$

Since for every $\alpha \in H^{m}(\Omega ;[0,1])$ and every $e_{1}, e_{2} \in L^{2}\left(\Omega ; \mathbb{M}_{s y m}^{n \times n}\right)$

$$
\mathcal{Q}\left(\alpha, e_{1}\right)-\mathcal{Q}\left(\alpha, e_{2}\right)=\frac{1}{2}\left\langle\mathbb{C}(\alpha)\left(e_{1}+e_{2}\right), e_{1}-e_{2}\right\rangle,
$$

recalling the integration-by-parts formula (1.20) and condition (b) of Lemma 2.1, by (2.19) we infer that

$$
\begin{aligned}
\mathcal{H}\left(\alpha_{k}^{i+1}, p_{k}^{i+1}-p_{k}^{i}\right) \leq & \left\langle\sigma_{k}^{i+1}, E\left(w_{k}^{i+1}-w_{k}^{i}\right)-\left(e_{k}^{i+1}-e_{k}^{i}\right)\right\rangle+\mathcal{Q}\left(\alpha_{k}^{i+1}, E\left(w_{k}^{i+1}-w_{k}^{i}\right)\right) \\
& -\left\langle\mathbb{C}\left(\alpha_{k}^{i+1}\right)\left(e_{k}^{i+1}-e_{k}^{i}\right), E\left(w_{k}^{i+1}-w_{k}^{i}\right)\right\rangle+\mathcal{Q}\left(\alpha_{k}^{i+1}, e_{k}^{i+1}-e_{k}^{i}\right) \\
= & \left\langle\left(\sigma_{k}^{i+1}\right)_{D} \mid p_{k}^{i+1}-p_{k}^{i}\right\rangle+\mathcal{Q}\left(\alpha_{k}^{i+1}, E\left(w_{k}^{i+1}-w_{k}^{i}\right)\right) \\
& -\left\langle\mathbb{C}\left(\alpha_{k}^{i+1}\right)\left(e_{k}^{i+1}-e_{k}^{i}\right), E\left(w_{k}^{i+1}-w_{k}^{i}\right)\right\rangle+\mathcal{Q}\left(\alpha_{k}^{i+1}, e_{k}^{i+1}-e_{k}^{i}\right) .
\end{aligned}
$$

By (2.21), using (1.15) and the Cauchy inequality we have

$$
r\left\|p_{k}^{i+1}-p_{k}^{i}\right\|_{1} \leq C_{1}\left(\left\|E\left(w_{k}^{i+1}-w_{k}^{i}\right)\right\|_{2}+\left\|e_{k}^{i+1}-e_{k}^{i}\right\|_{2}\right),
$$

where $C_{1}$ depends on $\gamma_{2}$ introduced in (1.7c) and on the constant in (2.10).

Testing (2.4) by $\left(u_{k}^{i+1}-\left(w_{k}^{i+1}-w_{k}^{i}\right), e_{k}^{i+1}-E\left(w_{k}^{i+1}-w_{k}^{i}\right), p_{k}^{i+1}\right) \in A\left(w_{k}^{i}\right)$, by simple algebraic manipulations we obtain

$$
\mathcal{Q}\left(\alpha_{k}^{i}, e_{k}^{i}\right)+\mathcal{Q}\left(\alpha_{k}^{i}, e_{k}^{i+1}-e_{k}^{i}\right)+\left\langle\sigma_{k}^{i}, E\left(w_{k}^{i+1}-w_{k}^{i}\right)\right\rangle \leq \mathcal{Q}\left(\alpha_{k}^{i}, e_{k}^{i+1}\right)+\mathcal{H}\left(\alpha_{k}^{i}, p_{k}^{i+1}-p_{k}^{i}\right) .
$$

Using (2.19), it follows that

$$
\begin{aligned}
\mathcal{Q}\left(\alpha_{k}^{i}, e_{k}^{i+1}-e_{k}^{i}\right) \leq & \mathcal{Q}\left(\alpha_{k}^{i}, e_{k}^{i+1}\right)-\mathcal{Q}\left(\alpha_{k}^{i}, e_{k}^{i}\right)+\mathcal{Q}\left(\alpha_{k}^{i+1}, e_{k}^{i}\right)-\mathcal{Q}\left(\alpha_{k}^{i+1}, e_{k}^{i+1}\right) \\
& +\left\langle\left[\mathbb{C}\left(\alpha_{k}^{i+1}\right)-\mathbb{C}\left(\alpha_{k}^{i}\right)\right] e_{k}^{i}, E\left(w_{k}^{i+1}-w_{k}^{i}\right)\right\rangle+\mathcal{Q}\left(\alpha_{k}^{i+1}, E\left(w_{k}^{i+1}-w_{k}^{i}\right)\right) \\
& +\mathcal{H}\left(\alpha_{k}^{i}, p_{k}^{i+1}-p_{k}^{i}\right)-\mathcal{H}\left(\alpha_{k}^{i+1}, p_{k}^{i+1}-p_{k}^{i}\right) .
\end{aligned}
$$

Notice now that, employing again (2.20),

$$
\mathcal{Q}\left(\alpha_{k}^{i}, e_{k}^{i+1}\right)-\mathcal{Q}\left(\alpha_{k}^{i}, e_{k}^{i}\right)+\mathcal{Q}\left(\alpha_{k}^{i+1}, e_{k}^{i}\right)-\mathcal{Q}\left(\alpha_{k}^{i+1}, e_{k}^{i+1}\right)=\frac{1}{2}\left\langle\left[\mathbb{C}\left(\alpha_{k}^{i}\right)-\mathbb{C}\left(\alpha_{k}^{i+1}\right)\right]\left(e_{k}^{i}+e_{k}^{i+1}\right),\left(e_{k}^{i+1}-e_{k}^{i}\right)\right\rangle .
$$

By $(2.23),(2.24),(1.7 \mathrm{c}),(1.16)$, and the Cauchy inequality, we deduce

$$
\begin{aligned}
\gamma_{1}\left\|e_{k}^{i+1}-e_{k}^{i}\right\|_{2}^{2} \leq & \frac{\gamma_{1}}{4}\left\|e_{k}^{i+1}-e_{k}^{i}\right\|_{2}^{2}+\frac{\gamma_{1} r^{2}}{4\left(C_{1}\right)^{2}}\left\|p_{k}^{i+1}-p_{k}^{i}\right\|_{1}^{2} \\
& +C_{2}\left(\left\|\alpha_{k}^{i+1}-\alpha_{k}^{i}\right\|_{\infty}^{2}+\left\|E\left(w_{k}^{i+1}-w_{k}^{i}\right)\right\|_{2}^{2}\right),
\end{aligned}
$$

with $C_{2}$ depending on the constant in (2.10), $w, \operatorname{Lip}(\mathbb{C}), C_{K}, r, \gamma_{1}, \gamma_{2}, \kappa$. Thus (2.17a) and (2.17b) follow from (2.22) and (2.25). Arguing as in [5, Theorem 3.8], we obtain also (2.17c) and (2.17d). Finally, using (2.17) and the Cauchy inequality, we get (2.18) from (2.21).

Combining Lemma 2.6 and some arguments from [18, Proposition 4.1], we prove that for $\varepsilon$ fixed the functions $\alpha_{k}$ are bounded in $H^{1}\left(0, T ; H^{m}(\Omega)\right)$, uniformly in $k$.

Proposition 2.7. There exists a positive constant $C$ independent of $\varepsilon, k$, and $t$ such that for every $\varepsilon>0$, $k \in \mathbb{N}, t \in(0, T) \backslash\left\{t_{k}^{1}, \ldots, t_{k}^{k-1}\right\}$

$$
\begin{gathered}
\varepsilon\left\|\dot{\alpha}_{k}(t)\right\|_{2} \leq C e^{\frac{C}{\varepsilon} \bar{\tau}_{k}(t)}, \\
\varepsilon \int_{0}^{\bar{\tau}_{k}(t)}\left\|\dot{\alpha}_{k}(s)\right\|_{m, 2}^{2} \mathrm{~d} s \leq C e^{\frac{C}{\varepsilon} \bar{\tau}_{k}(t)} .
\end{gathered}
$$

Proof. We start from (2.14), denoting $\dot{\alpha}_{k}^{i}:=\frac{\alpha_{k}^{i}-\alpha_{k}^{i-1}}{\tau}, \dot{e}_{k}^{i}=\frac{e_{k}^{i}-e_{k}^{i-1}}{\tau}, \dot{p}_{k}^{i}=\frac{p_{k}^{i}-p_{k}^{i-1}}{\tau}$, and $\dot{w}_{k}^{i}=\frac{w_{k}^{i}-w_{k}^{i-1}}{\tau}$ for every $k \in \mathbb{N}$ and $1 \leq i \leq k$. Let now fix $k$ and $i$. First we consider the case $2 \leq i \leq k$; the case $i=1$ needs a slightly different treatment and will be considered below.

We take (2.14b) in the case $\lambda=0$, respectively (2.15b) in the case $\lambda \in(0,1]$, evaluated at $t \in\left(t_{k}^{i-1}, t_{k}^{i}\right)$, thus $\bar{\alpha}_{k}(t)=\alpha_{k}^{i}$ and $\dot{\alpha}_{k}(t)=\dot{\alpha}_{k}^{i}$. Then we subtract (2.14a) (resp. (2.15a)) evaluated at $t \in\left(t_{k}^{i-2}, t_{k}^{i-1}\right)$, (thus $\bar{\alpha}_{k}(t)=\alpha_{k}^{i-1}$ and $\dot{\alpha}_{k}(t)=\dot{\alpha}_{k}^{i-1}$ ), and tested by $\beta:=\dot{\alpha}_{k}^{i}$. Recall that the differentiability of 
$\mathcal{E}_{\lambda}(\cdot, e ; p, t)$ follows from (1.13), which is assumed if $\lambda \in(0,1]$, while for $\lambda=0$ the energy reduces to $\mathcal{E}(\alpha, e)$ and some terms disappear, see also above. We obtain that for every $2 \leq i \leq k$ and every $\lambda \in[0,1]$

$$
\begin{aligned}
\varepsilon\left\langle\dot{\alpha}_{k}^{i}-\dot{\alpha}_{k}^{i-1}, \dot{\alpha}_{k}^{i}\right\rangle+\kappa\left\langle\alpha_{k}^{i}-\alpha_{k}^{i-1}, \dot{\alpha}_{k}^{i}\right\rangle_{m, 2} \leq & -\left[\frac{1}{2}\left\langle\mathbb{C}^{\prime}\left(\alpha_{k}^{i}\right) \dot{\alpha}_{k}^{i} e_{k}^{i}, e_{k}^{i}\right\rangle-\frac{1}{2}\left\langle\mathbb{C}^{\prime}\left(\alpha_{k}^{i-1}\right) \dot{\alpha}_{k}^{i} e_{k}^{i-1}, e_{k}^{i-1}\right\rangle\right] \\
& -\left\langle\partial D\left(\alpha_{k}^{i}\right)-\partial D\left(\alpha_{k}^{i-1}\right), \dot{\alpha}_{k}^{i}\right\rangle+C_{K} \tau\left\|\dot{\alpha}_{k}^{i}\right\|_{\infty}\left\|\dot{p}_{k}^{i}\right\|_{1} \\
& -\lambda \sum_{h=1}^{i-1}\left\langle\partial_{\alpha} \mathcal{H}\left(\alpha_{k}^{i}, p_{k}^{h}-p_{k}^{h-1}\right)-\partial_{\alpha} \mathcal{H}\left(\alpha_{k}^{i-1}, p_{k}^{h}-p_{k}^{h-1}\right), \dot{\alpha}_{k}^{i}\right\rangle .
\end{aligned}
$$

When $\lambda \in(0,1]$ the inequality stated above follows from (1.26) and (2.16) by neglecting the term $(1-\lambda)\left\langle\partial_{\alpha} \mathcal{H}\left(\alpha_{k}^{i-1}, p_{k}^{i-1}-p_{k}^{i-2}\right), \dot{\alpha}_{k}^{i}\right\rangle$, which is negative by the softening assumption (1.11b) and by the monotonicity in time of $\alpha_{k}$. Therefore

$$
\begin{aligned}
\varepsilon\left\langle\dot{\alpha}_{k}^{i}-\dot{\alpha}_{k}^{i-1}, \dot{\alpha}_{k}^{i}\right\rangle+\kappa\left\langle\alpha_{k}^{i}-\alpha_{k}^{i-1}, \dot{\alpha}_{k}^{i}\right\rangle_{m, 2} \leq & \frac{1}{2}\left|\left\langle\left[\mathbb{C}^{\prime}\left(\alpha_{k}^{i}\right)-\mathbb{C}^{\prime}\left(\alpha_{k}^{i-1}\right)\right] \dot{\alpha}_{k}^{i} e_{k}^{i}, e_{k}^{i}\right\rangle\right| \\
& +\frac{1}{2}\left|\left\langle\mathbb{C}^{\prime}\left(\alpha_{k}^{i-1}\right) \dot{\alpha}_{k}^{i} e_{k}^{i}, e_{k}^{i}\right\rangle-\left\langle\mathbb{C}^{\prime}\left(\alpha_{k}^{i-1}\right) \dot{\alpha}_{k}^{i} e_{k}^{i-1}, e_{k}^{i-1}\right\rangle\right| \\
& +C \tau\left\|\dot{\alpha}_{k}^{i}\right\|_{\infty}^{2}\left(1+\mathcal{V}\left(\bar{p}_{k} ; 0, \underline{\tau}_{k}(t)\right)\right)+C_{K} \tau\left\|\dot{\alpha}_{k}^{i}\right\|_{\infty}\left\|\dot{p}_{k}^{i}\right\|_{1},
\end{aligned}
$$

taking into account the regularity assumptions on $\mathbb{C}, D, H$ (see (1.7), (1.5), (1.12), (1.13)). Using the fact that $2 a(a-b) \geq a^{2}-b^{2}$ for every $a, b$, we get

$$
\varepsilon\left\langle\dot{\alpha}_{k}^{i}-\dot{\alpha}_{k}^{i-1}, \dot{\alpha}_{k}^{i}\right\rangle \geq \frac{\varepsilon}{2}\left(\left\|\dot{\alpha}_{k}^{i}\right\|_{2}^{2}-\left\|\dot{\alpha}_{k}^{i-1}\right\|_{2}^{2}\right)
$$

and then, using (2.12), we obtain that

$$
\begin{aligned}
\frac{\varepsilon}{2}\left(\left\|\dot{\alpha}_{k}^{i}\right\|_{2}^{2}-\left\|\dot{\alpha}_{k}^{i-1}\right\|_{2}^{2}\right)+\tau \kappa\left|\dot{\alpha}_{k}^{i}\right|_{m, 2}^{2} & \leq C \tau\left(\left\|\dot{\alpha}_{k}^{i}\right\|_{\infty}^{2}+\left\|\dot{\alpha}_{k}^{i}\right\|_{\infty}\left\|\dot{e}_{k}^{i}\right\|_{2}+\left\|\dot{\alpha}_{k}^{i}\right\|_{\infty}\left\|\dot{p}_{k}^{i}\right\|_{1}\right) \\
& \leq C \tau\left(\left\|\dot{\alpha}_{k}^{i}\right\|_{\infty}^{2}+\left\|E \dot{w}_{k}^{i}\right\|_{2}^{2}\right)
\end{aligned}
$$

for every $2 \leq i \leq k$, where $C$ depends on the $C^{1,1}$ norm of $\mathbb{C}, D, H$ (if $\lambda \in(0,1]$ ), and on the constants $r, \gamma_{1}, \gamma_{2}$. Notice that in the last inequality we have used Lemma 2.6.

Since $\partial_{\alpha} \mathcal{E}\left(\alpha_{0}, e_{0}\right) \in L^{2}(\Omega)$, using $(2.14 \mathrm{~b})$ we get

$$
\begin{aligned}
\varepsilon\left\|\dot{\alpha}_{k}^{1}\right\|_{2}^{2} \leq & \left\langle-\partial_{\alpha} \mathcal{E}\left(\alpha_{0}, e_{0}\right), \dot{\alpha}_{k}^{1}\right\rangle-\left[\frac{1}{2}\left(\left\langle\mathbb{C}^{\prime}\left(\alpha_{k}^{1}\right) \dot{\alpha}_{k}^{1} e_{k}^{1}, e_{k}^{1}\right\rangle-\left\langle\mathbb{C}^{\prime}\left(\alpha_{0}\right) \dot{\alpha}_{k}^{1} e_{0}, e_{0}\right\rangle_{L^{2}}\right)\right. \\
& \left.+\left\langle\partial D\left(\alpha_{k}^{1}\right)-\partial D\left(\alpha_{0}\right), \dot{\alpha}_{k}^{1}\right\rangle+\kappa\left\langle\alpha_{k}^{1}-\alpha_{0}, \dot{\alpha}_{k}^{1}\right\rangle_{m, 2}+C_{K} \tau\left\|\dot{p}_{k}^{1}\right\|_{1}\left\|\dot{\alpha}_{k}^{1}\right\|_{\infty}\right] \\
\leq & \frac{1}{2 \varepsilon}\left\|\partial_{\alpha} \mathcal{E}\left(\alpha_{0}, e_{0}\right)\right\|_{2}^{2}+\frac{\varepsilon}{2}\left\|\dot{\alpha}_{k}^{1}\right\|_{2}^{2}-\tau \kappa\left|\dot{\alpha}_{k}^{1}\right|_{m, 2}^{2}+C \tau\left(\left\|\dot{\alpha}_{k}^{1}\right\|_{\infty}^{2}+\left\|E \dot{w}_{k}^{1}\right\|_{2}^{2}\right),
\end{aligned}
$$

arguing as before, since $\left(\alpha_{0},\left(u_{0}, e_{0}, p_{0}\right)\right)$ satisfies (1.28). We can read (2.28) as

$$
\frac{\varepsilon}{2}\left\|\dot{\alpha}_{k}^{1}\right\|_{2}^{2}+\tau \kappa\left|\dot{\alpha}_{k}^{1}\right|_{m, 2}^{2} \leq C\left(\frac{1}{\varepsilon}+\tau\left(\left\|\dot{\alpha}_{k}^{1}\right\|_{\infty}^{2}+\left\|E \dot{w}_{k}^{1}\right\|_{2}^{2}\right)\right)
$$

Since $H^{m}(\Omega)$ is compactly embedded into $L^{\infty}(\Omega)$, for every $\delta>0$ there exists a constant $C_{\delta}>0$ such that

$$
\|\cdot\|_{\infty}^{2} \leq \delta|\cdot|_{m, 2}^{2}+C_{\delta}\|\cdot\|_{2}^{2}
$$

For every $2 \leq h \leq k$, summing (2.27) for $2 \leq i \leq h$ and (2.29) and taking into account (2.30) for $\delta=\frac{1}{2 C}$, we get

$$
\frac{\varepsilon}{2}\left\|\dot{\alpha}_{k}^{h}\right\|_{2}^{2}+\frac{\tau \kappa}{2} \sum_{i=1}^{h}\left|\dot{\alpha}_{k}^{i}\right|_{m, 2}^{2} \leq C\left(\frac{1}{\varepsilon}+\tau\left(\sum_{i=1}^{h}\left\|\dot{\alpha}_{k}^{i}\right\|_{2}^{2}+\sum_{i=1}^{h}\left\|E \dot{w}_{k}^{i}\right\|_{2}^{2}\right)\right) .
$$

Adding $\frac{\tau \kappa}{2} \sum_{i=1}^{h}\left\|\dot{\alpha}_{k}^{i}\right\|_{2}^{2}$ to both sides of (2.31), it follows that for every $t \in(0, T) \backslash\left\{t_{k}^{1}, \ldots, t_{k}^{k-1}\right\}$,

$$
\begin{aligned}
\frac{\varepsilon}{2}\left\|\dot{\alpha}_{k}(t)\right\|_{2}^{2}+\frac{\kappa}{2} \int_{0}^{\bar{\tau}_{k}(t)}\left\|\dot{\alpha}_{k}(s)\right\|_{m, 2}^{2} \mathrm{~d} s & \leq C\left(\frac{1}{\varepsilon}+\int_{0}^{\bar{\tau}_{k}(t)}\left\|\dot{\alpha}_{k}(s)\right\|_{2}^{2} \mathrm{~d} s+\int_{0}^{\bar{\tau}_{k}(t)}\left\|E \dot{w}_{k}(s)\right\|_{2}^{2} \mathrm{~d} s\right) \\
& \leq C\left(\frac{1}{\varepsilon}+1+\int_{0}^{\bar{\tau}_{k}(t)}\left\|\dot{\alpha}_{k}(s)\right\|_{2}^{2} \mathrm{~d} s\right),
\end{aligned}
$$

where we have used (1.27) in the last inequality. Now Gronwall's Inequality implies that

$$
\varepsilon\left\|\dot{\alpha}_{k}(t)\right\|_{2}^{2} \leq C\left(\frac{1}{\varepsilon}+1\right) e^{\frac{C}{\varepsilon} \bar{\tau}_{k}(t)}
$$

for every $t \in(0, T) \backslash\left\{t_{k}^{1}, \ldots, t_{k}^{k-1}\right\}$. We recover (2.26a) multiplying with $\varepsilon$ and taking the square root. Now (2.26b) follows from (2.26a) and (2.32). 
Arguing as in [18, Proposition 4.3] we improve the estimate of Proposition 2.7 and show that the functions $\alpha_{k}$ are bounded in $A C\left([0, T], H^{m}(\Omega)\right)$ by a constant independent of $\varepsilon$ and $k$.

Proposition 2.8. There exists a positive constant $C$ independent of $\varepsilon, k$, and $t$ such that for every $0<\varepsilon<1$ and $k \in \mathbb{N}$, with $k \geq(4 \varepsilon)^{-1}$,

$$
\int_{0}^{t}\left\|\dot{\alpha}_{k}(s)\right\|_{m, 2} \mathrm{~d} s \leq C
$$

Proof. Let $\dot{\alpha}_{k}^{i}:=\frac{\alpha_{k}^{i}-\alpha_{k}^{i-1}}{\tau}$ and $\dot{w}_{k}^{i}=\frac{w_{k}^{i}-w_{k}^{i-1}}{\tau}$ for every $k \in \mathbb{N}$ and $1 \leq i \leq k$. From the inequality

$$
\left\|\dot{\alpha}_{k}^{i}\right\|_{2}\left(\left\|\dot{\alpha}_{k}^{i}\right\|_{2}-\left\|\dot{\alpha}_{k}^{i-1}\right\|_{2}\right) \leq\left\langle\dot{\alpha}_{k}^{i}-\dot{\alpha}_{k}^{i-1}, \dot{\alpha}_{k}^{i}\right\rangle_{2},
$$

arguing as done for (2.27), we get that for every $2 \leq i \leq k$

$$
\varepsilon\left\|\dot{\alpha}_{k}^{i}\right\|_{2}\left(\left\|\dot{\alpha}_{k}^{i}\right\|_{2}-\left\|\dot{\alpha}_{k}^{i-1}\right\|_{2}\right)+\tau \kappa\left|\dot{\alpha}_{k}^{i}\right|_{m, 2}^{2} \leq C \tau\left(\left\|\dot{\alpha}_{k}^{i}\right\|_{\infty}^{2}+\left\|E \dot{w}_{k}^{i}\right\|_{2}^{2}\right) .
$$

By the compact embedding of $H^{m}(\Omega)$ into $L^{\infty}(\Omega)$, for every $\delta>0$ there exists a constant $C(\delta)>0$ such that

$$
\|\cdot\|_{\infty}^{2} \leq \delta|\cdot|_{m, 2}^{2}+C_{\delta}\|\cdot\|_{1}^{2} \leq \delta|\cdot|_{m, 2}^{2}+\widetilde{C}_{\delta}\|\cdot\|_{1}\|\cdot\|_{2},
$$

since $\Omega$ is bounded. Adding a term $\tau \kappa\left\|\dot{\alpha}_{k}^{i}\right\|_{2}^{2}$ to both sides of (2.33) and using (2.34) with $\delta=\frac{1}{2 C}$, we obtain that

$$
\varepsilon\left\|\dot{\alpha}_{k}^{i}\right\|_{2}\left(\left\|\dot{\alpha}_{k}^{i}\right\|_{2}-\left\|\dot{\alpha}_{k}^{i-1}\right\|_{2}\right)+\frac{\kappa}{2} \tau\left\|\dot{\alpha}_{k}^{i}\right\|_{m, 2}^{2} \leq C \tau\left(\left\|\dot{\alpha}_{k}^{i}\right\|_{1}\left\|\dot{\alpha}_{k}^{i}\right\|_{2}+\left\|E \dot{w}_{k}^{i}\right\|_{2}^{2}\right),
$$

for $2 \leq i \leq k$. Multiplying the inequality above by $2 / \varepsilon$ and taking into account that $\left\|\dot{\alpha}_{k}^{i}\right\|_{m, 2}^{2} \geq\left\|\dot{\alpha}_{k}^{i}\right\|_{2}^{2}$, we have that

$$
2\left\|\dot{\alpha}_{k}^{i}\right\|_{2}\left(\left\|\dot{\alpha}_{k}^{i}\right\|_{2}-\left\|\dot{\alpha}_{k}^{i-1}\right\|_{2}\right)+\frac{\tau \kappa}{2 \varepsilon}\left\|\dot{\alpha}_{k}^{i}\right\|_{2}^{2}+\frac{\tau \kappa}{2 \varepsilon}\left\|\dot{\alpha}_{k}^{i}\right\|_{m, 2}^{2} \leq \frac{2 \tau C}{\varepsilon}\left(\left\|\dot{\alpha}_{k}^{i}\right\|_{1}\left\|\dot{\alpha}_{k}^{i}\right\|_{2}+\left\|E \dot{w}_{k}^{i}\right\|_{2}^{2}\right)
$$

for $2 \leq i \leq k$. We now set

$$
a_{i}:=\left\|\dot{\alpha}_{k}^{i}\right\|_{2}, \quad b_{i}:=\left(\frac{\tau \kappa}{2 \varepsilon}\right)^{1 / 2}\left\|\dot{\alpha}_{k}^{i}\right\|_{m, 2}, \quad c_{i}:=\left(\frac{2 \tau C}{\varepsilon}\right)^{1 / 2}\left\|E \dot{w}_{k}^{i}\right\|_{2} \quad d_{i}:=\frac{\tau C}{\varepsilon}\left\|\dot{\alpha}_{k}^{i}\right\|_{1} \quad \zeta:=\frac{\tau \kappa}{4 \varepsilon}
$$

for $2 \leq i \leq k$. This definition allows us to recast (2.35) in the form

$$
2 a_{i}\left(a_{i}-a_{i-1}\right)+2 \zeta a_{i}^{2}+b_{i}^{2} \leq c_{i}^{2}+2 a_{i} d_{i}
$$

and so to follow the proof performed in [18]. Indeed, by a discrete Gronwall-type inequality with weights (see [18, Lemma 4.1], to which we refer for all details), we conclude that

$$
\begin{aligned}
\frac{\kappa}{2 \varepsilon} \sum_{i=2}^{h} \tau(1+\zeta)^{2(i-h)-1}\left\|\dot{\alpha}_{k}^{i}\right\|_{m, 2}^{2} \leq & 2(1+\zeta)^{-2 h}\left\|\dot{\alpha}_{k}^{1}\right\|_{2}^{2}+16 C \zeta \sum_{i=2}^{h}(1+\zeta)^{2(i-h)-1}\left\|E \dot{w}_{k}^{i}\right\|_{2}^{2} \\
& +4 C^{2}\left(\sum_{i=2}^{h} \frac{\tau}{\varepsilon}(1+\zeta)^{i-h-1}\left\|\dot{\alpha}_{k}^{i}\right\|_{1}\right)^{2},
\end{aligned}
$$

for $2 \leq h \leq k$. We bound the right hand side of (2.36) with

$$
\left[\sqrt{2}(1+\zeta)^{-h}\left\|\dot{\alpha}_{k}^{1}\right\|_{2}+\left(1+16 C \zeta \sum_{i=2}^{k}(1+\zeta)^{2(i-h)-1}\left\|E \dot{w}_{k}^{i}\right\|_{2}^{2}\right)+2 C \sum_{i=2}^{h} \frac{\tau}{\varepsilon}(1+\zeta)^{i-h-1}\left\|\dot{\alpha}_{k}^{i}\right\|_{1}\right]^{2},
$$

using the fact that for every $a, b, c>0$

$$
a^{2}+b^{2}+c^{2} \leq(a+b+c)^{2} \leq\left(a+\left(1+b^{2}\right)+c\right)^{2} .
$$

In order to estimate from below the left hand side of (2.36), we appeal to the Hölder inequality,

$$
\frac{\kappa}{2 \varepsilon} \sum_{i=2}^{h} \tau(1+\zeta)^{2(i-h)-1}\left\|\dot{\alpha}_{k}^{i}\right\|_{m, 2} \leq\left(\frac{\kappa}{2 \varepsilon} \sum_{i=2}^{h} \tau(1+\zeta)^{2(i-h)-1}\right)^{\frac{1}{2}}\left(\frac{\kappa}{2 \varepsilon} \sum_{i=2}^{h} \tau(1+\zeta)^{2(i-h)-1}\left\|\dot{\alpha}_{k}^{i}\right\|_{m, 2}^{2}\right)^{\frac{1}{2}} .
$$

Evaluating the geometric sum and using the fact that $\frac{\tau \kappa}{2 \varepsilon} \leq 2 \zeta \leq 2 \kappa$, we deduce that

$$
\frac{1}{2 \varepsilon} \sum_{i=2}^{h} \tau(1+\zeta)^{2(i-h)-1} \leq \kappa+1
$$


Therefore from (2.36) we obtain

$$
\begin{aligned}
\frac{1}{\varepsilon} \sum_{i=2}^{h} \tau(1+\zeta)^{2(i-h)-1}\left\|\dot{\alpha}_{k}^{i}\right\|_{m, 2} \leq C( & +(1+\zeta)^{-h}\left\|\dot{\alpha}_{k}^{1}\right\|_{2}+\zeta \sum_{i=2}^{h}(1+\zeta)^{2(i-h)-1}\left\|E \dot{w}_{k}^{i}\right\|_{2}^{2} \\
& \left.+\sum_{i=2}^{h} \frac{\tau}{\varepsilon}(1+\zeta)^{i-h-1}\left\|\dot{\alpha}_{k}^{i}\right\|_{1}\right) .
\end{aligned}
$$

Now we multiply both sides of (2.37) by $\tau$ and sum over $h=2, \ldots, k$. Recalling the formula of the geometric sum we get for every $k \in \mathbb{N}$ and $\rho_{2}, \rho_{3}, \ldots, \rho_{k} \geq 0$

$$
\frac{1}{\varepsilon} \sum_{h=2}^{k} \tau \sum_{i=2}^{h} \tau(1+\zeta)^{2(i-h)-1} \rho_{i}=4 \frac{1+\zeta}{2+\zeta} \sum_{i=2}^{k} \tau \rho_{i}\left(1-(1+\zeta)^{2(i-k)-2}\right),
$$

where we have changed the order of the sums; this identity will be used to rewrite the left hand side of (2.37) setting $\rho_{i}=\left\|\dot{\alpha}_{k}^{i}\right\|_{m, 2}$ and the third term in the right hand side setting $\rho_{i}=\left\|E \dot{w}_{k}^{i}\right\|_{2}^{2}$. Moreover, for the second and the fourth summand in the right hand side of (2.37) we have

$$
\sum_{h=2}^{k} \tau(1+\zeta)^{-h}\left\|\dot{\alpha}_{k}^{1}\right\|_{2} \leq \frac{8 \varepsilon}{\kappa}\left\|\dot{\alpha}_{k}^{1}\right\|_{2}
$$

and

$$
\sum_{h=2}^{k} \tau \sum_{i=2}^{h} \frac{\tau}{\varepsilon}(1+\zeta)^{i-h-1}\left\|\dot{\alpha}_{k}^{i}\right\|_{1} \leq \frac{4}{\kappa} \sum_{i=2}^{k} \tau\left\|\dot{\alpha}_{k}^{i}\right\|_{1} .
$$

Collecting (2.37)-(2.40) we obtain that

$$
\sum_{i=2}^{k} \tau\left\|\dot{\alpha}_{k}^{i}\right\|_{m, 2} \leq C\left(T+\varepsilon\left\|\dot{\alpha}_{k}^{1}\right\|_{2}+\sum_{i=2}^{k} \tau\left\|E \dot{w}_{k}^{i}\right\|_{2}^{2}+\sum_{i=2}^{k} \tau\left\|\dot{\alpha}_{k}^{i}\right\|_{1}\right)+\sum_{i=2}^{k} \tau(1+\zeta)^{2(i-k)-2}\left\|\dot{\alpha}_{k}^{i}\right\|_{m, 2} .
$$

The last term in the equation above is estimated with (2.37), so we get

$$
\sum_{i=2}^{k} \tau\left\|\dot{\alpha}_{k}^{i}\right\|_{m, 2} \leq C\left(T+\varepsilon\left\|\dot{\alpha}_{k}^{1}\right\|_{2}+\sum_{i=2}^{k} \tau\left\|E \dot{w}_{k}^{i}\right\|_{2}^{2}+\sum_{i=2}^{k} \tau\left\|\dot{\alpha}_{k}^{i}\right\|_{1}\right)
$$

We are now left to estimate the term with $i=1$. From (2.29) and (2.34) it follows that

$$
\tau\left|\dot{\alpha}_{k}^{1}\right|_{m, 2}^{2} \leq C\left(\frac{1}{\varepsilon}+\tau\left\|\dot{\alpha}_{k}^{1}\right\|_{1}^{2}+\tau\left\|E \dot{w}_{k}^{1}\right\|_{2}^{2}\right) .
$$

Multiplying by $\tau$, since $\frac{\tau}{2 \varepsilon} \leq 2$ we get

$$
\tau^{2}\left|\dot{\alpha}_{k}^{1}\right|_{m, 2}^{2} \leq C\left(1+\tau^{2}\left\|\dot{\alpha}_{k}^{1}\right\|_{1}^{2}+\tau^{2}\left\|E \dot{w}_{k}^{1}\right\|_{2}^{2}\right) \leq C\left(1+\tau\left\|\dot{\alpha}_{k}^{1}\right\|_{1}+\tau\left\|E \dot{w}_{k}^{1}\right\|_{2}\right)^{2},
$$

and then

$$
\tau\left\|\dot{\alpha}_{k}^{1}\right\|_{m, 2} \leq C\left(1+\tau\left\|\dot{\alpha}_{k}^{1}\right\|_{1}+\tau\left\|E \dot{w}_{k}^{1}\right\|_{2}\right)+\tau\left\|\dot{\alpha}_{k}^{1}\right\|_{2} \leq C\left(1+\tau\left\|\dot{\alpha}_{k}^{1}\right\|_{1}+\tau\left\|E \dot{w}_{k}^{1}\right\|_{2}+\varepsilon\left\|\dot{\alpha}_{k}^{1}\right\|_{2}\right) .
$$

Summing up (2.41) and (2.42) gives

$$
\begin{aligned}
\sum_{i=1}^{k} \tau\left\|\dot{\alpha}_{k}^{i}\right\|_{m, 2} & \leq C\left(T+\varepsilon\left\|\dot{\alpha}_{k}^{1}\right\|_{2}+\sum_{i=1}^{k} \tau\left\|E \dot{w}_{k}^{i}\right\|_{2}^{2}+\sum_{i=1}^{k} \tau\left\|\dot{\alpha}_{k}^{i}\right\|_{1}\right) \\
& \leq C\left(1+\int_{0}^{T}\left\|E \dot{w}_{k}(s)\right\|_{2}^{2}+\int_{\Omega} \alpha_{0}(x)-\alpha_{k}^{k}(x) \mathrm{d} x\right)
\end{aligned}
$$

where in the last inequality we have used the fact that $\alpha_{k}^{i} \leq \alpha_{k}^{i-1}$ and (2.26a) for $t \in\left(0, t_{k}^{1}\right)=(0, \tau)$, taking into account that $\frac{\tau}{\varepsilon} \leq 4$. Thus we conclude, recalling (1.27), (2.11b), and the fact that $C$ is independent of $\varepsilon, k$, and $t$.

Remark 2.9. Using Lemma 2.6, by Proposition 2.7 we get that

$$
\varepsilon \int_{0}^{T}\left\|\dot{e}_{k}(s)\right\|_{2}^{2} \mathrm{~d} s \leq C, \quad \varepsilon \int_{0}^{T}\left\|\dot{p}_{k}(s)\right\|_{1}^{2} \mathrm{~d} s \leq C, \quad \varepsilon \int_{0}^{T}\left\|\dot{u}_{k}(s)\right\|_{B D}^{2} \mathrm{~d} s \leq C,
$$

while by Proposition 2.8 it follows that

$$
\int_{0}^{T}\left\|\dot{e}_{k}(s)\right\|_{2} \mathrm{~d} s \leq C, \quad \int_{0}^{T}\left\|\dot{p}_{k}(s)\right\|_{1} \mathrm{~d} s \leq C, \quad \int_{0}^{T}\left\|\dot{u}_{k}(s)\right\|_{B D} \mathrm{~d} s \leq C
$$

for $4 k \varepsilon>1$, where $C$ is a constant independent of $\varepsilon, k$, and $t$. 
Remark 2.10. We conclude this section with a short discussion on the choice of the regularizing term in (1.9). Let us consider the general case of a damage regularization in a Banach space $X$, namely, whenever the energy is finite, the damage variable belongs to $X$. In order to differentiate the energy with respect to time, a priori estimates for $\alpha_{k}$ in the space $W^{1,1}(0, T ; X)$ should be derived, in such a way that $\dot{\alpha}$ is in duality with $\partial_{\alpha} \mathcal{E} \in X^{\prime}$, cf. (1.10). In the present context, following [18], we exploit the Hilbert structure of the space $X=H^{m}(\Omega)$, see Lemma 2.8. Instead, the choice $X=W^{1, \gamma}(\Omega)(\gamma>n)$ considered in [17] (for damage without plasticity) provides only a uniform estimate for $\alpha_{k}$ in $W^{1,1}\left(0, T ; H^{1}(\Omega)\right.$ ). For this reason in [17] the evolution fulfills only an energy inequality, see also Remark 3.4 therein.

\section{Viscous EVOLUtions}

In this section we pass to the limit in the discrete-time problems as the time step converges to zero. For every fixed $\varepsilon>0$ we then find a quadruple $\left(\alpha_{\varepsilon}, u_{\varepsilon}, e_{\varepsilon}, p_{\varepsilon}\right)$ satisfying for every $t \in[0, T]$ :

- admissibility and equilibrium conditions, with $\alpha_{\varepsilon}$ nonincreasing in time;

- a first order stability condition in the damage, referred to as Kuhn-Tucker inequality;

- an energy balance including viscous dissipation.

Such quadruples are called $\varepsilon$-approximate viscous evolutions (see Definition 3.1 and Theorem 3.7). We also prove some crucial estimates for the passage to the limit as viscosity vanishes, which will be studied in Section 4.

We start introducing the notion of $\varepsilon$-approximate viscous evolution. Notice that when $X$ is the dual space of a Banach space $Y$ we denote

$$
L_{w}^{2}(0, T ; X):=\left\{p:[0, T] \rightarrow X \text { weakly* measurable }: t \mapsto\|p(t)\| \in L^{2}(0, T)\right\},
$$

with $f:(0, T) \rightarrow X$ weakly* measurable if and only if $(0, T) \ni t \mapsto\langle f(t), g\rangle$ is measurable for every $g \in Y$, and

$$
\begin{gathered}
H_{w}^{1}(0, T ; X):=\left\{p \in L_{w}^{2}(0, T ; X): \exists \widetilde{p} \in L_{w}^{2}(0, T ; X) \text { s.t. for every } \varphi \in C_{c}^{1}((0, T) ; Y)\right. \\
\left.\int_{0}^{T}\left\langle p(t), \partial_{t} \varphi(t)\right\rangle \mathrm{d} t=-\int_{0}^{T}\langle\widetilde{p}(t), \varphi(t)\rangle \mathrm{d} t\right\} .
\end{gathered}
$$

Definition 3.1. Let (1.5), (1.7), (1.11) hold, and let $w$ be as in (1.27). We say that a function $\left(\alpha_{\varepsilon}, u_{\varepsilon}, e_{\varepsilon}, p_{\varepsilon}\right)$ from $[0, T]$ into $H^{m}(\Omega ;[0,1]) \times B D(\Omega) \times L^{2}\left(\Omega ; \mathbb{M}_{s y m}^{n \times n}\right) \times M_{b}\left(\Omega \cup \partial_{D} \Omega ; \mathbb{M}_{D}^{n \times n}\right)$ is an $\varepsilon-$ approximate viscous evolution if

$$
\begin{aligned}
& \alpha_{\varepsilon} \in H^{1}\left(0, T ; H^{m}(\Omega)\right), \quad e_{\varepsilon} \in H^{1}\left(0, T ; L^{2}\left(\Omega ; \mathbb{M}_{s y m}^{n \times n}\right)\right), \\
& u_{\varepsilon} \in H_{w}^{1}(0, T ; B D(\Omega)), \quad p_{\varepsilon} \in H_{w}^{1}\left(0, T ; M_{b}\left(\Omega \cup \partial_{D} \Omega ; \mathbb{M}_{D}^{n \times n}\right)\right),
\end{aligned}
$$

and, setting $\sigma_{\varepsilon}(t):=\mathbb{C}\left(\alpha_{\varepsilon}(t)\right) e_{\varepsilon}(t)$ for every $t \in[0, T]$, the following conditions are satisfied:

$(\mathrm{ev} 0)_{\varepsilon}$ irreversibility: for every $x \in \Omega$

$$
[0, T] \ni t \mapsto \alpha_{\varepsilon}(t, x) \text { is nonincreasing; }
$$

$(\mathrm{ev} 1)_{\varepsilon}$ kinematic condition and equilibrium: for every $t \in[0, T]$

$$
\left(u_{\varepsilon}(t), e_{\varepsilon}(t), p_{\varepsilon}(t)\right) \in A(w(t)), \quad \operatorname{div} \sigma_{\varepsilon}(t)=0 \text { in } \Omega, \quad\left[\sigma_{\varepsilon} \nu\right]=0 \text { on } \partial_{N} \Omega ;
$$

$(\mathrm{ev} 2)_{\varepsilon}$ stress constraint: for every $t \in[0, T]$

$$
\sigma_{\varepsilon}(t) \in \mathcal{K}_{\alpha_{\varepsilon}(t)}(\Omega)
$$

$(\mathrm{ev} 3)_{\varepsilon}$ Kuhn-Tucker inequality: for a.e. $t \in(0, T)$

$$
\left\langle\partial_{\alpha} \mathcal{E}_{\lambda}\left(\alpha_{\varepsilon}(t), e_{\varepsilon}(t) ; p_{\varepsilon}, t\right), \beta\right\rangle+\varepsilon\left\langle\dot{\alpha}_{\varepsilon}(t), \beta\right\rangle \geq 0 \quad \text { for every } \beta \in H_{-}^{m}(\Omega)=\left\{\beta \in H^{m}(\Omega): \beta \leq 0 \text { in } \Omega\right\} ;
$$

$(\mathrm{ev} 4)_{\varepsilon}$ energy balance: for every $t \in[0, T]$

$$
\begin{aligned}
& \mathcal{E}_{\lambda}\left(\alpha_{\varepsilon}(t), e_{\varepsilon}(t) ; p_{\varepsilon}, t\right)+(1-\lambda) \int_{0}^{t} \mathcal{H}\left(\alpha_{\varepsilon}(s), \dot{p}_{\varepsilon}(s)\right) \mathrm{d} s+\varepsilon \int_{0}^{t}\left\|\dot{\alpha}_{\varepsilon}(s)\right\|_{2}^{2} \mathrm{~d} s \\
& \quad=\mathcal{E}\left(\alpha_{0}, e_{0}\right)+\int_{0}^{t}\left\langle\sigma_{\varepsilon}(s), E \dot{w}(s)\right\rangle \mathrm{d} s .
\end{aligned}
$$

Two characterizations of the approximate viscous evolutions are given below: the first ensures in particular that the damage variable satisfies the Kuhn-Tucker conditions, while the second will be useful in the proof of Theorem 3.7 .

Proposition 3.2. Let $\left(\alpha_{\varepsilon}, u_{\varepsilon}, e_{\varepsilon}, p_{\varepsilon}\right)$ be a function satisfying the conditions $(3.1)$, $(\operatorname{ev0})_{\varepsilon}-(\operatorname{ev} 3)_{\varepsilon}$, with $\alpha_{\varepsilon}(t) \in H^{m}(\Omega ;[0,1])$. Then $\left(\alpha_{\varepsilon}, u_{\varepsilon}, e_{\varepsilon}, p_{\varepsilon}\right)$ is an $\varepsilon$-approximate viscous evolution, i.e. it satisfies the energy balance $(\mathrm{ev} 4)_{\varepsilon}$, if and only if any of the conditions below holds true: 
$\left(\mathrm{ev} 4{ }^{\prime}\right)_{\varepsilon}$ for a.e. $t \in(0, T)$ the following hold:

- Kuhn-Tucker equality:

$$
\left\langle\partial_{\alpha} \mathcal{E}_{\lambda}\left(\alpha_{\varepsilon}(t), e_{\varepsilon}(t) ; p_{\varepsilon}, t\right), \dot{\alpha}_{\varepsilon}(t)\right\rangle+\varepsilon\left\|\dot{\alpha}_{\varepsilon}(t)\right\|_{2}^{2}=0 ;
$$

- Hill's maximum plastic work principle:

$$
\mathcal{H}\left(\alpha_{\varepsilon}(t), \dot{p}_{\varepsilon}(t)\right)=\left\langle\sigma_{\varepsilon}(t)\right)_{D}\left|\dot{p}_{\varepsilon}(t)\right\rangle .
$$

$(\mathrm{ev} 4 ")_{\varepsilon}$ energy inequality: for every $t \in[0, T]$

$$
\begin{aligned}
& \mathcal{E}_{\lambda}\left(\alpha_{\varepsilon}(T), e_{\varepsilon}(T) ; p_{\varepsilon}, T\right)+(1-\lambda) \int_{0}^{T} \mathcal{H}\left(\alpha_{\varepsilon}(t), \dot{p}_{\varepsilon}(t)\right) \mathrm{d} t+\varepsilon \int_{0}^{T}\left\|\dot{\alpha}_{\varepsilon}(t)\right\|_{2}^{2} \mathrm{~d} t \\
& \quad \leq \mathcal{E}\left(\alpha_{0}, e_{0}\right)+\int_{0}^{T}\left\langle\sigma_{\varepsilon}(t), E \dot{w}(t)\right\rangle \mathrm{d} t .
\end{aligned}
$$

Proof. Ad $(\mathrm{ev} 4)_{\varepsilon} \Longleftrightarrow\left(\mathrm{ev} 4^{\prime}\right)_{\varepsilon}$ : From the absolute continuity of $\alpha_{\varepsilon}, e_{\varepsilon}$, and $p_{\varepsilon}$, we obtain that the function $t \mapsto \mathcal{E}_{\lambda}\left(\alpha_{\varepsilon}(t), e_{\varepsilon}(t) ; p_{\varepsilon}, t\right)$ is absolutely continuous and

$$
\frac{\mathrm{d}}{\mathrm{d} t} \mathcal{E}_{\lambda}\left(\alpha_{\varepsilon}(t), e_{\varepsilon}(t) ; p_{\varepsilon}, t\right)=\left\langle\partial_{\alpha} \mathcal{E}_{\lambda}\left(\alpha_{\varepsilon}(t), e_{\varepsilon}(t) ; p_{\varepsilon}, t\right), \dot{\alpha}_{\varepsilon}(t)\right\rangle+\lambda \mathcal{H}\left(\alpha_{\varepsilon}(t), \dot{p}_{\varepsilon}(t)\right)+\left\langle\sigma_{\varepsilon}(t), \dot{e}_{\varepsilon}(t)\right\rangle
$$

for a.e. $t \in(0, T)$. Property $(\mathrm{ev} 1)_{\varepsilon}$ and [5, Lemma 5.5] imply that

$$
\left(\dot{u}_{\varepsilon}(t), \dot{e}_{\varepsilon}(t), \dot{p}_{\varepsilon}(t)\right) \in A(\dot{w}(t)) \text { for a.e. } t \in(0, T),
$$

so that, from the integration by parts formula (1.20),

$$
\left\langle\sigma_{\varepsilon}(t), \dot{e}_{\varepsilon}(t)\right\rangle=\left\langle\sigma_{\varepsilon}(t), E \dot{w}(t)\right\rangle-\left\langle\left(\sigma_{\varepsilon}(t)\right)_{D} \mid \dot{p}_{\varepsilon}(t)\right\rangle
$$

for a.e. $t \in(0, T)$. Then $(\mathrm{ev} 4)_{\varepsilon}$ is equivalent to

$$
-(1-\lambda) \mathcal{H}\left(\alpha_{\varepsilon}(t), \dot{p}_{\varepsilon}(t)\right)-\varepsilon\left\|\dot{\alpha}_{\varepsilon}(t)\right\|_{2}^{2}+\left\langle\sigma_{\varepsilon}(t), E \dot{w}(t)\right\rangle=\frac{\mathrm{d}}{\mathrm{d} t} \mathcal{E}_{\lambda}\left(\alpha_{\varepsilon}(t), e_{\varepsilon}(t) ; p_{\varepsilon}, t\right)
$$

for a.e. $t \in(0, T)$, which is also equivalent to

$$
\left\langle\partial_{\alpha} \mathcal{E}_{\lambda}\left(\alpha_{\varepsilon}(t), e_{\varepsilon}(t) ; p_{\varepsilon}, t\right), \dot{\alpha}_{\varepsilon}(t)\right\rangle+\varepsilon\left\|\dot{\alpha}_{\varepsilon}(t)\right\|_{2}^{2}+\mathcal{H}\left(\alpha_{\varepsilon}(t), \dot{p}_{\varepsilon}(t)\right)-\left\langle\left(\sigma_{\varepsilon}(t)\right)_{D} \mid \dot{p}_{\varepsilon}(t)\right\rangle=0
$$

for a.e. $t \in(0, T)$. Now, from $(\mathrm{ev} 2)_{\varepsilon}$ and $(1.22)$ it follows that

$$
\left\langle\left(\sigma_{\varepsilon}(t)\right)_{D} \mid \dot{p}_{\varepsilon}(t)\right\rangle \leq \mathcal{H}\left(\alpha_{\varepsilon}(t), \dot{p}_{\varepsilon}(t)\right),
$$

since $\dot{p}_{\varepsilon}(t) \in \Pi(\Omega)$ for a.e. $t \in[0, T]$. Then, using (ev3) $)_{\varepsilon}$ with $\beta=\dot{\alpha}_{\varepsilon}$, we get that (3.5) is equivalent to $\left(\mathrm{ev} 4^{\prime}\right)_{\varepsilon}$.

Ad $(\mathrm{ev} 4)_{\varepsilon} \Longleftrightarrow(\text { ev4" })_{\varepsilon}$ : Let us prove that $(\mathrm{ev} 4 ")_{\varepsilon}$ implies $(\mathrm{ev} 4)_{\varepsilon}$, the converse being trivial. Gathering $(\mathrm{ev} 3)_{\varepsilon}$ with $\beta=\dot{\alpha}_{\varepsilon}(t),(3.3),(3.4)$, and (3.6), we deduce that

$$
\frac{\mathrm{d}}{\mathrm{d} t} \mathcal{E}_{\lambda}\left(\alpha_{\varepsilon}(t), e_{\varepsilon}(t) ; p_{\varepsilon}, t\right) \geq-(1-\lambda) \mathcal{H}\left(\alpha_{\varepsilon}(t), \dot{p}_{\varepsilon}(t)\right)-\varepsilon\left\|\dot{\alpha}_{\varepsilon}(t)\right\|_{2}^{2}+\left\langle\sigma_{\varepsilon}(t), E \dot{w}(t)\right\rangle
$$

for a.e. $t \in(0, T)$. Integrating, we get for every $0 \leq t_{1} \leq t_{2} \leq T$ the inequality

$$
\begin{aligned}
& \mathcal{E}_{\lambda}\left(\alpha_{\varepsilon}\left(t_{2}\right), e_{\varepsilon}\left(t_{2}\right) ; p_{\varepsilon}, t_{2}\right)+(1-\lambda) \int_{t_{1}}^{t_{2}} \mathcal{H}\left(\alpha_{\varepsilon}(s), \dot{p}_{\varepsilon}(s)\right) \mathrm{d} s+\varepsilon \int_{t_{1}}^{t_{2}}\left\|\dot{\alpha}_{\varepsilon}(s)\right\|_{2}^{2} \mathrm{~d} s \\
& \quad \geq \mathcal{E}_{\lambda}\left(\alpha_{\varepsilon}\left(t_{1}\right), e_{\varepsilon}\left(t_{1}\right) ; p_{\varepsilon}, t_{1}\right)+\int_{t_{1}}^{t_{2}}\left\langle\sigma_{\varepsilon}(s), E \dot{w}(s)\right\rangle \mathrm{d} s,
\end{aligned}
$$

which implies the energy balance $(\mathrm{ev} 4)_{\varepsilon}$ in view of $(\mathrm{ev} 4 ")_{\varepsilon}$. This concludes the proof.

Using the Kuhn-Tucker conditions, we can rewrite the energy balance as in the following Remark.

Remark 3.3. Let $\left(\alpha_{\varepsilon}, u_{\varepsilon}, e_{\varepsilon}, p_{\varepsilon}\right)$ be an $\varepsilon$-approximate viscous evolution. From (ev3) and (3.2) it follows that

$$
\varepsilon\left\|\dot{\alpha}_{\varepsilon}(t)\right\|_{2}=\sup _{\beta \in F}\left\langle-\partial_{\alpha} \mathcal{E}_{\lambda}\left(\alpha_{\varepsilon}(t), e_{\varepsilon}(t) ; p_{\varepsilon}, t\right), \beta\right\rangle=-\inf _{\beta \in F}\left\langle\partial_{\alpha} \mathcal{E}_{\lambda}\left(\alpha_{\varepsilon}(t), e_{\varepsilon}(t) ; p_{\varepsilon}, t\right), \beta\right\rangle
$$

for a.e. $t \in(0, T)$, where

Indeed, by $(\mathrm{ev} 3)_{\varepsilon}$

$$
F:=\left\{\beta \in H_{-}^{m}(\Omega):\|\beta\|_{2} \leq 1\right\}
$$

$$
\varepsilon\left\langle\dot{\alpha}_{\varepsilon}(t), \beta\right\rangle \geq\left\langle-\partial_{\alpha} \mathcal{E}_{\lambda}\left(\alpha_{\varepsilon}(t), e_{\varepsilon}(t) ; p_{\varepsilon}, t\right), \beta\right\rangle,
$$

for every $\beta \in H_{-}^{m}(\Omega)$, while (3.2) implies that the supremum in (3.7) is a maximum, attained for $\beta=\frac{\dot{\alpha}_{\varepsilon}(t)}{\left\|\dot{\alpha}_{\varepsilon}(t)\right\|_{2}}$ if $\left\|\dot{\alpha}_{\varepsilon}(t)\right\|_{2}>0$. 
Then, by $(3.7),(\text { ev4 })_{\varepsilon}$ reads as

$$
\begin{aligned}
& \mathcal{E}_{\lambda}\left(\alpha_{\varepsilon}(T), e_{\varepsilon}(T) ; p_{\varepsilon}, T\right)+(1-\lambda) \int_{0}^{T} \mathcal{H}\left(\alpha_{\varepsilon}(t), \dot{p}_{\varepsilon}(t)\right) \mathrm{d} t+\int_{0}^{T}\left\|\dot{\alpha}_{\varepsilon}(t)\right\|_{2} \Psi\left(\alpha_{\varepsilon}(t), e_{\varepsilon}(t) ; p_{\varepsilon}, t\right) \mathrm{d} t \\
& \quad=\mathcal{E}\left(\alpha_{0}, e_{0}\right)+\int_{0}^{T}\left\langle\sigma_{\varepsilon}(t), E \dot{w}(t)\right\rangle \mathrm{d} t,
\end{aligned}
$$

where

$$
\Psi(\alpha, e ; p, t):=\Phi\left(\partial_{\alpha} \mathcal{E}_{\lambda}(\alpha, e ; p, t)\right),
$$

for every $\alpha \in H^{m}(\Omega ;[0,1]), e \in L^{2}\left(\Omega ; \mathbb{M}_{\text {sym }}^{n \times n}\right), p \in A C\left([0, T] ; M_{b}\left(\Omega \cup \partial_{D} \Omega ; \mathbb{M}_{D}^{n \times n}\right)\right), t \in[0, T]$, with

$$
\Phi(f):=\sup _{\beta \in F}\langle-f, \beta\rangle \quad \text { for every } f \in\left(H^{m}(\Omega)\right)^{\prime} .
$$

Notice that $\Psi(\alpha, e ; p, t) \in[0,+\infty]$.

In the following lemma we characterize the operator $\Phi$ introduced above.

Lemma 3.4. Let $\Phi$ be the operator defined in (3.10), and let

$$
G:=\left\{h \in\left(H^{m}(\Omega)\right)^{\prime}:\langle h, \beta\rangle \geq 0 \quad \text { for every } \beta \in H_{-}^{m}(\Omega)\right\}
$$

and

$$
\mathrm{d}_{2}(f, G):=\min \left\{\|g\|_{2}: g \in L^{2}(\Omega), f+g \in G\right\} \quad \text { for every } f \in\left(H^{m}(\Omega)\right)^{\prime},
$$

which is well defined for every $f$. Then

$$
\Phi(f)=\mathrm{d}_{2}(f, G) \quad \text { for every } f \in\left(H^{m}(\Omega)\right)^{\prime} .
$$

Proof. Let us fix $f \in\left(H^{m}(\Omega)\right)^{\prime}$.

Proof of $\Phi(f) \leq \mathrm{d}_{2}(f, G)$. Let $\mathrm{d}_{2}(f, G)<+\infty$ and $g \in L^{2}(\Omega)$ such that $f+g \in G$ : we have that

$$
\langle-f, \beta\rangle \leq \int_{\Omega} g \beta \mathrm{d} x \leq\|g\|_{2} \quad \text { for every } \beta \in F
$$

and we conclude by definition of $\Phi(f)$ and $\mathrm{d}_{2}(f, G)$.

Proof of $\mathrm{d}_{2}(f, G) \leq \Phi(f)$. We can assume $\Phi(f)<+\infty$; then

$$
\langle f, \beta\rangle \leq \Phi(f)\|\beta\|_{2} \quad \text { for every } \beta \in H_{+}^{m}(\Omega)=\left\{\beta \in H^{m}(\Omega): \beta \geq 0 \text { in } \Omega\right\} \text {. }
$$

Let $B \subset \mathbb{R}^{n}$ be an open set such that $\bar{\Omega} \subset B$ and

$$
\langle S, \beta\rangle:=\left\langle f,\left.\beta\right|_{\Omega}\right\rangle \quad \text { for every } \beta \in H_{0}^{m}(B) ;
$$

by $(3.13)$

$$
\langle S, \beta\rangle \leq \Phi(f)\|\beta\|_{L^{2}(B)} \quad \text { for every } \beta \in H_{0}^{m}(B) .
$$

By Lemma A.2 we get that there exists a unique pair $(g, \mu)$ with $g \in L^{2}(B), g \geq 0$ and $\mu \in M^{+}(B)$ (namely $\mu$ is a nonnegative measure on $B$ ) such that $g \mathrm{~d} x$ and $\mu$ are mutually singular and

$$
\langle S, \beta\rangle=\int_{B} g \beta \mathrm{d} x-\int_{B} \beta \mathrm{d} \mu \quad \text { for every } \beta \in H_{0}^{m}(B) ;
$$

in particular the former property implies that $\int_{B} g \mathrm{~d} \mu^{a}=0$.

Using (3.14) we have that $g \in L^{2}(\Omega)$ and $\mu \in M^{+}(\bar{\Omega})$. Therefore

$$
\langle f, \beta\rangle=\int_{\Omega} g \beta \mathrm{d} x-\int_{\bar{\Omega}} \beta \mathrm{d} \mu \quad \text { for every } \beta \in H^{m}(\Omega) ;
$$

then $-\langle g, \cdot\rangle_{2}+f \in G$, and this gives

$$
\mathrm{d}_{2}(f, G) \leq\|g\|_{2} .
$$

Let us fix $\varepsilon>0$. We claim that there exists $\beta \in C_{c}^{\infty}(\Omega) \cap F$ such that

$$
-\int_{\Omega} g \beta \mathrm{d} x>\|g\|_{2}-\varepsilon \text { and }-\int_{\Omega} \beta \mathrm{d} \mu^{a}<\varepsilon,
$$

where $\mu=\mu^{a}+\mu^{s}$ is the decomposition of $\mu \in M^{+}(\bar{\Omega})$ into its absolutely continuous and its singular part (with respect to $\mathcal{L}^{n}$ ). Indeed, we can first consider $h \in L^{\infty}(\Omega)$ with compact support such that $-\frac{g}{\|g\|_{2}} \leq h \leq 0$ and

$$
-\int_{\Omega} g h \mathrm{~d} x>\|g\|_{2}-\frac{\varepsilon}{2}
$$


for instance $h=\left(-1_{\Omega_{k}} \frac{g}{\|g\|_{2}}\right) \vee(-k)$ for $\Omega_{k}$ compact such that $\Omega \subset \Omega_{k}+B\left(0, \frac{1}{k}\right)$ and $k \in \mathbb{N}$ large enough. Then we set $h_{k}:=\frac{h * \varrho_{k}}{1 \vee\left\|h * \varrho_{k}\right\|_{2}}$ for a suitable $k \in \mathbb{N}$ (here $\rho_{k}(t):=k \rho\left(\frac{t}{k}\right)$, with $\rho$ the standard mollifier in $\mathbb{R})$, so that $h_{k} \in F,\left\|h_{k}\right\|_{\infty} \leq\|h\|_{\infty}$ for every $k$, and

$$
\lim _{k \rightarrow \infty} \int_{\Omega} h_{k} \mathrm{~d} \mu^{a}=\int_{\Omega} h \mathrm{~d} \mu^{a}=0
$$

where the first equality follows by Dominated Convergence Theorem and the second from $-\frac{g}{\|g\|_{2}} \leq h \leq 0$ and $\int_{\Omega} g \mathrm{~d} \mu^{a}=0$. Since $h_{k} \rightarrow h$ in $L^{2}(\Omega)$, for $k$ large $\left\|h-h_{k}\right\|_{2}<\frac{\varepsilon}{2\|g\|_{2}}$ and then we get (3.16) by (3.17) and (3.18).

Let us now consider $\mu^{s} \in M^{+}(\bar{\Omega})$; let $E$ be the set on which $\mu^{s}$ is concentrated, and $K$ be a compact subset of $E$ such that

$$
\mu^{s}(E \backslash K)<\frac{\varepsilon}{\|\beta\|_{\infty}} .
$$

Since $\mathcal{L}^{n}(E)=0$, for every $\eta>0$ we can find an open set $U$ such that $K \subset U$ and $\mathcal{L}^{n}(U)<\eta$. Let us take $\varphi \in C_{c}^{\infty}(\Omega ;[0,1])$ such that $\varphi=0$ in $K$ and $\varphi=1$ in $\Omega \backslash U$, and let $\bar{\beta}:=\beta \varphi$. Then $\bar{\beta} \in F$ and we can assume that $\bar{\beta}$ satisfies (3.16), choosing $\eta$ sufficiently small: then

$$
\Phi(f) \geq-\langle f, \bar{\beta}\rangle=-\int_{\Omega} g \bar{\beta}+\int_{\Omega} \bar{\beta} \mathrm{d} \mu^{a}+\int_{E \backslash K} \bar{\beta} \mathrm{d} \mu^{s}>\|g\|_{2}-3 \varepsilon \geq \mathrm{d}_{2}(f, G)-3 \varepsilon,
$$

by (3.15), (3.16), and (3.19). The proof is concluded since $\varepsilon$ is arbitrary.

Remark 3.5. The identity (3.12) can be used to connect the notions of solutions provided in [18] and in [28], in the context of damage (without plasticity). The energy balance has the same structure for the two evolutions; the term related to the energy dissipated during jumps in the energy balance of [18] is given in terms of $d_{2}(\cdot, G)$, while the one in [28] is given in terms of $\Phi$.

The following Lemma states some semicontinuity properties that will be useful for the proof of Theorem 3.7. For the reader's convenience we give the proof following the lines of [7, Lemmas 6.1 and 6.2$]$, to which we refer for full details.

Lemma 3.6. Let $\beta_{k}, \beta \in C([0, T] ; C(\bar{\Omega} ;[0,1]))$ such that

$$
\beta_{k} \rightarrow \beta \text { in } C([0, T] ; C(\bar{\Omega})),
$$

and $q_{k}, q \in H^{1}\left(0, T ; M_{b}\left(\Omega \cup \partial_{D} \Omega ; \mathbb{M}_{D}^{n \times n}\right)\right)$ such that

$$
q_{k}(t) \stackrel{*}{\rightarrow} q(t) \quad \text { in } M_{b}\left(\Omega \cup \partial_{D} \Omega ; \mathbb{M}_{D}^{n \times n}\right)
$$

for every $t \in[0, T]$ and

$$
\int_{0}^{T}\left\|\dot{q}_{k}(t)\right\|_{1} \mathrm{~d} t+\int_{0}^{T}\|\dot{q}(t)\|_{1} \mathrm{~d} t \leq C
$$

for $C$ independent of $k$. Then for every $t \in[0, T]$

$$
\int_{0}^{t} \mathcal{H}(\beta(s), \dot{q}(s)) \mathrm{d} s \leq \liminf _{k \rightarrow \infty} \int_{0}^{t} \mathcal{H}\left(\beta_{k}(s), \dot{q}_{k}(s)\right) \mathrm{d} s
$$

and

$$
\left\langle\partial_{\alpha} \widehat{\mathcal{V}}_{\mathcal{H}}\left(\beta_{1}, q ; 0, t\right), \beta_{2}\right\rangle \leq \liminf _{k \rightarrow \infty}\left[\left\langle\partial_{\alpha} \widehat{\mathcal{V}}_{\mathcal{H}}\left(\beta_{1}, q_{k} ; 0, t\right), \beta_{2}\right\rangle\right]
$$

for every $\beta_{1} \in C(\bar{\Omega} ;[0,1])$ and $\beta_{2} \in C(\bar{\Omega} ;[0, \infty))$.

Proof. Let us fix $t \in[0, T]$ and define $\mu_{k}, \mu \in M_{b}\left((0, t) \times\left(\Omega \cup \partial_{D} \Omega\right) ; \mathbb{M}_{D}^{n \times n}\right)$ by setting

$$
\left\langle\varphi, \mu_{k}\right\rangle:=\int_{0}^{t}\left\langle\varphi(s, \cdot), \dot{q}_{k}(s)\right\rangle \mathrm{d} s \text { and }\langle\varphi, \mu\rangle:=\int_{0}^{t}\langle\varphi(s, \cdot), \dot{q}(s)\rangle \mathrm{d} s
$$

for every $\varphi \in C_{0}\left((0, t) \times\left(\Omega \cup \partial_{D} \Omega\right) ; \mathbb{M}_{D}^{n \times n}\right)$. Using (3.21) and (3.22) it is possible to see that

$$
\mu_{k} \rightarrow \mu \quad \text { weakly* in } M_{b}\left((0, t) \times\left(\Omega \cup \partial_{D} \Omega\right) ; \mathbb{M}_{D}^{n \times n}\right)
$$

by uniform approximation, cf. [7, Lemma 6.1].

Since $s \mapsto|\dot{q}(s)|$ is weakly* measurable from $(0, t)$ into $M_{b}\left(\Omega \cup \partial_{D} \Omega ; \mathbb{M}_{D}^{n \times n}\right)$, we define $\nu_{k}, \nu \in$ $M_{b}\left((0, t) \times\left(\Omega \cup \partial_{D} \Omega\right)\right)$ by

$$
\left\langle\varphi, \nu_{k}\right\rangle:=\int_{0}^{t}\left\langle\varphi(s, \cdot),\left|\dot{q}_{k}(s)\right|\right\rangle \mathrm{d} s \text { and }\langle\varphi, \nu\rangle:=\int_{0}^{t}\langle\varphi(s, \cdot),|\dot{q}(s)|\rangle \mathrm{d} s
$$


for every $\varphi \in C_{0}\left((0, t) \times\left(\Omega \cup \partial_{D} \Omega\right)\right)$. As in [7, Lemma 6.1], we have that $\mu_{k} \ll \nu_{k}, \mu \ll \nu$ and

$$
\begin{aligned}
\int_{0}^{t} \mathcal{H}\left(\beta(s), \dot{q}_{k}(s)\right) \mathrm{d} s & =\int_{(0, t) \times\left(\Omega \cup \partial_{D} \Omega\right)} H\left(\beta(s, x), \frac{\mathrm{d} \mu_{k}}{\mathrm{~d} \nu_{k}}(s, x)\right) \mathrm{d} \nu_{k}(s, x), \\
\int_{0}^{t} \mathcal{H}(\beta(s), \dot{q}(s)) \mathrm{d} s & =\int_{(0, t) \times\left(\Omega \cup \partial_{D} \Omega\right)} H\left(\beta(s, x), \frac{\mathrm{d} \mu}{\mathrm{d} \nu}(s, x)\right) \mathrm{d} \nu(s, x) .
\end{aligned}
$$

By Reshetnyak's Lower Semicontinuity Theorem and (3.25), we have

$$
\int_{0}^{t} \mathcal{H}(\beta(s), \dot{q}(s)) \mathrm{d} s \leq \liminf _{k \rightarrow \infty} \int_{0}^{t} \mathcal{H}\left(\beta(s), \dot{q}_{k}(s)\right) \mathrm{d} s .
$$

In order to get (3.23), it is enough to observe that (1.16) gives

$$
\left|\int_{0}^{t} \mathcal{H}\left(\beta_{k}(s), \dot{q}_{k}(s)\right) \mathrm{d} s-\int_{0}^{t} \mathcal{H}\left(\beta(s), \dot{q}_{k}(s)\right) \mathrm{d} s\right| \leq C_{K} \sup _{s \in[0, t]}\left\|\beta_{k}(s)-\beta(s)\right\|_{\infty} \int_{0}^{t}\left\|\dot{q}_{k}(s)\right\|_{1} \mathrm{~d} s,
$$

and the same holds replacing $q_{k}$ with $q$. Then we get (3.23) by (3.20), (3.22), and (3.26).

We can argue similarly to prove (3.24), noticing that

$$
\begin{aligned}
\left\langle\partial_{\alpha} \widehat{\mathcal{V}}_{\mathcal{H}}\left(\beta_{1}, q_{k} ; 0, t\right), \beta_{2}\right\rangle & =\int_{(0, t) \times\left(\Omega \cup \partial_{D} \Omega\right)} \partial_{\alpha} H\left(\beta_{1}(x), \frac{\mathrm{d} \mu_{k}}{\mathrm{~d} \nu_{k}}(s, x)\right) \beta_{2}(x) \mathrm{d} \nu_{k}(s, x), \\
\left\langle\partial_{\alpha} \widehat{\mathcal{V}}_{\mathcal{H}}\left(\beta_{1}, q ; 0, t\right), \beta_{2}\right\rangle & =\int_{(0, t) \times\left(\Omega \cup \partial_{D} \Omega\right)} \partial_{\alpha} H\left(\beta_{1}(x), \frac{\mathrm{d} \mu}{\mathrm{d} \nu}(s, x)\right) \beta_{2}(x) \mathrm{d} \nu(s, x),
\end{aligned}
$$

and applying Reshetnyak's Lower Semicontinuity Theorem, since $(x, \xi) \mapsto \partial_{\alpha} H\left(\beta_{1}(x), \xi\right) \beta_{2}(x)$ is a nonnegative continuous function positively 1-homogeneous and convex in the second variable. This allows us to conclude.

We prove now the existence of a family of absolutely continuous $\varepsilon$-approximate viscous evolutions according to Definition 3.1, satisfying in addition a uniform bound on the $L^{1}$-norm of the time derivative.

Theorem 3.7. Assume (1.5), (1.7), (1.11), (1.27), (1.28) for given $\alpha_{0}, u_{0}, e_{0}, p_{0}$ and, if $\lambda \in(0,1]$, also (1.13). There exists a family $\left\{\left(\alpha_{\varepsilon}, u_{\varepsilon}, e_{\varepsilon}, p_{\varepsilon}\right)\right\}_{\varepsilon>0}$ of $\varepsilon$-approximate viscous evolutions such that $\left(\alpha_{\varepsilon}(0), u_{\varepsilon}(0), e_{\varepsilon}(0), p_{\varepsilon}(0)\right)=\left(\alpha_{0}, u_{0}, e_{0}, p_{0}\right)$ and

$$
\int_{0}^{T}\left\|\dot{\alpha}_{\varepsilon}(t)\right\|_{m, 2} \mathrm{~d} t+\int_{0}^{T}\left\|\dot{e}_{\varepsilon}(t)\right\|_{2} \mathrm{~d} t \leq C
$$

with $C$ independent of $\varepsilon$.

Proof. The proof is divided in subsequent steps.

Time-discretization and time-continuous limit. Let us fix $\varepsilon>0$. Starting with the given initial condition $\left(\alpha_{0}, u_{0}, e_{0}, p_{0}\right)$ we consider the incremental problems $(2.1)$ in correspondence with the parameter $\varepsilon>0$, thus obtaining a sequence of approximate solutions

$$
\alpha_{k, \varepsilon} \equiv \alpha_{k}, \quad u_{k, \varepsilon} \equiv u_{k} \quad e_{k, \varepsilon} \equiv e_{k} \quad p_{k, \varepsilon} \equiv p_{k} .
$$

We use the same notation of Section 2 for their piecewise constant interpolants.

From (2.26b) we have

$$
\left\|\bar{\alpha}_{k}-\alpha_{k}\right\|_{L^{\infty}\left(0, T ; H^{m}(\Omega)\right)} \leq \tau^{1 / 2}\left\|\dot{\alpha}_{k}\right\|_{L^{2}\left(0, T ; H^{m}(\Omega)\right)} \leq C_{\varepsilon} \tau^{1 / 2},
$$

and the same holds for $u_{k}, e_{k}$, and $p_{k}$, by Remark 2.9. By standard compactness results and Helly's Theorem, there exist $\alpha_{\varepsilon} \in H^{1}\left(0, T ; H^{m}(\Omega)\right)$ and $e_{\varepsilon} \in H^{1}\left(0, T ; L^{2}\left(\Omega ; \mathbb{M}_{s y m}^{n \times n}\right)\right)$ such that (up to subsequences)

$$
\begin{gathered}
\alpha_{k} \rightarrow \alpha_{\varepsilon} \text { in } H^{1}\left(0, T ; H^{m}(\Omega)\right), \quad e_{k} \rightarrow e_{\varepsilon} \text { in } H^{1}\left(0, T ; L^{2}\left(\Omega ; \mathbb{M}_{\text {sym }}^{n \times n}\right)\right), \\
\alpha_{k}(t) \rightarrow \alpha_{\varepsilon}(t) \text { in } H^{m}(\Omega), \quad e_{k}(t) \rightarrow e_{\varepsilon}(t) \text { in } L^{2}\left(\Omega ; \mathbb{M}_{\text {sym }}^{n \times n}\right) \quad \text { for every } t \in[0, T],
\end{gathered}
$$

and

$$
\alpha_{k} \rightarrow \alpha_{\varepsilon} \text { in } C([0, T] ; C(\bar{\Omega})),
$$

since $H^{m}(\Omega)$ is compactly embedded into $C(\bar{\Omega})$.

In particular, since $\dot{\alpha}_{k} \rightarrow \dot{\alpha_{\varepsilon}}$ in $L^{1}\left(0, T ; H^{m}(\Omega)\right)$ and $\dot{e}_{k} \rightarrow \dot{e}_{\varepsilon}$ in $L^{1}\left(0, T ; L^{2}\left(\Omega ; \mathbb{M}_{s y m}^{n \times n}\right)\right)$, by Proposition 2.8 and (2.44) there exists a constant $C$ independent of $\varepsilon>0$ such that

$$
\int_{0}^{T}\left\|\dot{\alpha}_{\varepsilon}(t)\right\|_{m, 2} \mathrm{~d} t+\int_{0}^{T}\left\|\dot{e}_{\varepsilon}(t)\right\|_{2} \mathrm{~d} t \leq C
$$

for every $\varepsilon>0$. 
Taking into account (2.43) and the fact that $p_{k}(0)=p_{0}$ and $u_{k}(0)=u_{0}$ for every $k$, from Lemma A.1 it follows that there exist $p_{\varepsilon} \in H_{w}^{1}\left(0, T ; M_{b}\left(\Omega \cup \partial_{D} \Omega ; \mathbb{M}_{D}^{n \times n}\right)\right), u_{\varepsilon} \in H^{1}\left(0, T ; L^{q}\left(\Omega ; \mathbb{R}^{n}\right)\right)$, with $1 \leq q<\frac{n}{n-1}$, and $E_{\varepsilon} \in H_{w}^{1}\left(0, T ; M_{b}\left(\Omega ; \mathbb{M}_{s y m}^{n \times n}\right)\right)$ such that, for a suitable subsequence,

$$
\begin{gathered}
\dot{p}_{k} \stackrel{*}{\rightarrow} \dot{p} \text { in } L_{w}^{2}\left(0, T ; M_{b}\left(\Omega \cup \partial_{D} \Omega ; \mathbb{M}_{D}^{n \times n}\right)\right), \\
\dot{u}_{k} \rightarrow \dot{u}_{\varepsilon} \text { in } L^{2}\left(0, T ; L^{q}\left(\Omega ; \mathbb{R}^{n}\right)\right), \quad E \dot{u}_{k} \stackrel{*}{\rightarrow} \dot{E}_{\varepsilon} \text { in } L_{w}^{2}\left(0, T ; M_{b}\left(\Omega ; \mathbb{M}_{s y m}^{n \times n}\right)\right),
\end{gathered}
$$

and

$$
\begin{gathered}
p_{k}(t) \stackrel{*}{\rightarrow} p_{\varepsilon}(t) \text { in } M_{b}\left(\Omega \cup \partial_{D} \Omega ; \mathbb{M}_{D}^{n \times n}\right), \\
u_{k}(t) \rightarrow u_{\varepsilon}(t) \text { in } L^{1}\left(\Omega ; \mathbb{R}^{n}\right), \quad E u_{k}(t) \stackrel{*}{\rightarrow} E_{\varepsilon}(t) \text { in } M_{b}\left(\Omega ; \mathbb{M}_{s y m}^{n \times n}\right)
\end{gathered}
$$

for every $t \in[0, T]$. This implies that $E u_{\varepsilon}(t)=E_{\varepsilon}(t)$ for every $t \in[0, T]$, hence

$$
u_{\varepsilon} \in H_{w}^{1}(0, T ; B D(\Omega)), \quad u_{k}(t) \stackrel{*}{\rightarrow} u_{\varepsilon}(t) \text { in } B D(\Omega) \text { for every } t \in[0, T] .
$$

Let us now prove that $\left(\alpha_{\varepsilon}, u_{\varepsilon}, e_{\varepsilon}, p_{\varepsilon}\right)$ is an $\varepsilon$-approximate viscous evolution. The irreversibility condition $(\mathrm{ev0})_{\varepsilon}$ holds by (3.29) and the monotonicity in time of the $\alpha_{k}$. We can assume that (3.29), (3.31), and (3.32) hold for the same subsequence and thus $(\mathrm{ev} 1)_{\varepsilon}$ follows by [5, Lemma 2.1] and by the fact that $w_{k}(t) \rightarrow w(t)$ in $H^{1}\left(\mathbb{R}^{n} ; \mathbb{R}^{n}\right)$ for every $t\left(w\right.$ being continuous into $\left.H^{1}\left(\mathbb{R}^{n} ; \mathbb{R}^{n}\right)\right)$.

We now prove $(\mathrm{ev} 2)_{\varepsilon}$. Let us fix $t \in[0, T]$. For

$$
\widehat{\sigma}_{k}(t, x):=\Pi_{K\left(\alpha_{\varepsilon}(t, x)\right)}\left(\sigma_{k}(t, x)\right),
$$

$\Pi_{K\left(\alpha_{\varepsilon}(t, x)\right)}$ being the projection onto $K\left(\alpha_{\varepsilon}(t, x)\right)$, we have by (1.11c) that

$$
\left|\bar{\sigma}_{k}(t, x)-\widehat{\sigma}_{k}(t, x)\right| \leq C_{K}\left|\bar{\alpha}_{k}(t, x)-\alpha_{\varepsilon}(t, x)\right|
$$

for every $x$ such that $\bar{\sigma}_{k}(t, x) \in K\left(\bar{\alpha}_{k}(t, x)\right)$, and then

$$
\left\|\bar{\sigma}_{k}(t)-\widehat{\sigma}_{k}(t)\right\|_{\infty} \leq C_{K}\left\|\bar{\alpha}_{k}(t)-\alpha_{\varepsilon}(t)\right\|_{\infty} .
$$

We now recall that $\bar{\sigma}_{k}(t) \in \mathcal{K}_{\bar{\alpha}_{k}(t)}(\Omega), \bar{\sigma}_{k}(t) \rightarrow \sigma_{\varepsilon}(t)$ in $L^{2}\left(\Omega ; \mathbb{M}_{s y m}^{n \times n}\right)$, and $\bar{\alpha}_{k}(t) \rightarrow \alpha_{\varepsilon}(t)$ uniformly in $\bar{\Omega}$ for every $t \in[0, T]$. Therefore $\mathcal{K}_{\alpha_{\varepsilon}(t)}(\Omega) \ni \widehat{\sigma}_{k}(t) \rightarrow \sigma_{\varepsilon}(t)$ in $L^{2}\left(\Omega ; \mathbb{M}_{s y m}^{n \times n}\right)$ and $\sigma_{\varepsilon}(t) \in \mathcal{K}_{\alpha_{\varepsilon}(t)}(\Omega)$, by convexity of the sets $K(\alpha)$.

By Proposition 3.2, it is enough to prove the energy inequality $(\text { ev4" })_{\varepsilon}$ and the Kuhn-Tucker inequality $(\mathrm{ev} 3)_{\varepsilon}$.

Proof of the energy inequality $(\mathbf{e v 4 " )})_{\varepsilon}$. From the absolute continuity of $\alpha_{k}, e_{k}$, and $p_{k}$, we get that $t \mapsto \mathcal{E}_{\lambda}\left(\alpha_{k}(t), e_{k}(t) ; p_{k}, t\right)$ is absolutely continuous and for every $k \in \mathbb{N}, t \in(0, T) \backslash\left\{t_{k}^{1}, \ldots, t_{k}^{k-1}\right\}$,

$$
\frac{\mathrm{d}}{\mathrm{d} t} \mathcal{E}_{\lambda}\left(\alpha_{k}(t), e_{k}(t) ; p_{k}, t\right)=\left\langle\partial_{\alpha} \mathcal{E}_{\lambda}\left(\alpha_{k}(t), e_{k}(t) ; p_{k}, t\right), \dot{\alpha}_{k}(t)\right\rangle+\lambda \mathcal{H}\left(\alpha_{k}(t), \dot{p}_{k}(t)\right)+\left\langle\sigma_{k}(t), \dot{e}_{k}(t)\right\rangle .
$$

We first consider the case $\lambda \in(0,1]$. By (2.15b) and (2.16)

$$
\frac{\mathrm{d}}{\mathrm{d} t} \mathcal{E}_{\lambda}\left(\alpha_{k}(t), e_{k}(t) ; p_{k}, t\right)=-\varepsilon\left\|\dot{\alpha}_{k}(t)\right\|_{2}^{2}+\lambda \mathcal{H}\left(\alpha_{k}(t), \dot{p}_{k}(t)\right)+\left\langle\sigma_{k}(t), \dot{e}_{k}(t)\right\rangle+\delta_{k}(t),
$$

where

$$
\begin{aligned}
\delta_{k}(t):= & -(1-\lambda) \tau\left\langle\partial_{\alpha} \mathcal{H}\left(\bar{\alpha}_{k}(t), \dot{p}_{k}(t)\right), \dot{\alpha}_{k}(t)\right\rangle-\frac{1}{2}\left\langle\left[\mathbb{C}^{\prime}\left(\bar{\alpha}_{k}(t)\right)-\mathbb{C}^{\prime}\left(\alpha_{k}(t)\right)\right] \dot{\alpha}_{k}(t) e_{k}(t), e_{k}(t)\right\rangle \\
& -\frac{1}{2}\left[\left\langle\mathbb{C}^{\prime}\left(\bar{\alpha}_{k}(t)\right) \dot{\alpha}_{k}(t) \bar{e}_{k}(t), \bar{e}_{k}(t)\right\rangle-\left\langle\mathbb{C}^{\prime}\left(\bar{\alpha}_{k}(t)\right) \dot{\alpha}_{k}(t) e_{k}(t), e_{k}(t)\right\rangle\right] \\
& -\left\langle\partial D\left(\bar{\alpha}_{k}(t)\right)-\partial D\left(\alpha_{k}(t)\right), \dot{\alpha}_{k}(t)\right\rangle-\kappa\left\langle\bar{\alpha}_{k}(t)-\alpha_{k}(t), \dot{\alpha}_{k}(t)\right\rangle_{m, 2} \\
& -\lambda\left[\left\langle\partial_{\alpha} \widehat{\mathcal{V}}_{\mathcal{H}}\left(\bar{\alpha}_{k}(t), p_{k} ; 0, t\right), \dot{\alpha}_{k}(t)\right\rangle-\left\langle\partial_{\alpha} \widehat{\mathcal{V}}_{\mathcal{H}}\left(\alpha_{k}(t), p_{k} ; 0, t\right), \dot{\alpha}_{k}(t)\right\rangle\right] .
\end{aligned}
$$

Let us estimate $\delta_{k}(t)$. First remark that

$$
\begin{aligned}
& \int_{0}^{T}\left|\frac{1}{2}\left\langle\left[\mathbb{C}^{\prime}\left(\bar{\alpha}_{k}(t)\right)-\mathbb{C}^{\prime}\left(\alpha_{k}(t)\right)\right] \dot{\alpha}_{k}(t) e_{k}(t), e_{k}(t)\right\rangle\right|+\left|\left\langle\partial D\left(\bar{\alpha}_{k}(t)\right)-\partial D\left(\alpha_{k}(t)\right), \dot{\alpha}_{k}(t)\right\rangle\right| \\
& +\lambda\left|\left\langle\partial_{\alpha} \widehat{\mathcal{V}}_{\mathcal{H}}\left(\bar{\alpha}_{k}(t), p_{k} ; 0, t\right), \dot{\alpha}_{k}(t)\right\rangle-\left\langle\partial_{\alpha} \widehat{\mathcal{V}}_{\mathcal{H}}\left(\alpha_{k}(t), p_{k} ; 0, t\right), \dot{\alpha}_{k}(t)\right\rangle\right| \\
& +\kappa\left|\left\langle\bar{\alpha}_{k}(t)-\alpha_{k}(t), \dot{\alpha}_{k}(t)\right\rangle_{m, 2}\right| \mathrm{d} t \\
& \quad \leq C\left\|\bar{\alpha}_{k}-\alpha_{k}\right\|_{L^{\infty}\left(0, T ; H^{m}(\Omega)\right)} \int_{0}^{T}\left\|\dot{\alpha}_{k}(t)\right\|_{H^{m}(\Omega)},
\end{aligned}
$$

where $C$ depends on $D, \bar{C}_{K}, \kappa, \sup _{t}\left\|e_{k}(t)\right\|_{2}, \mathcal{V}_{\mathcal{H}}\left(p_{k} ; 0, T\right)$, and on the $C^{1,1}$ norm of $\mathbb{C}$. Moreover,

$$
\left\langle\partial_{\alpha} \mathcal{H}\left(\bar{\alpha}_{k}(t), \dot{p}_{k}(t)\right), \dot{\alpha}_{k}(t)\right\rangle \leq C\left(\left\|\dot{\alpha}_{k}(t)\right\|_{\infty}^{2}+\left\|\dot{p}_{k}(t)\right\|_{1}^{2}\right)
$$


and

$$
\begin{aligned}
& \int_{0}^{T}\left|\left\langle\mathbb{C}^{\prime}\left(\bar{\alpha}_{k}(t)\right) \dot{\alpha}_{k}(t) \bar{e}_{k}(t), \bar{e}_{k}(t)\right\rangle-\left\langle\mathbb{C}^{\prime}\left(\bar{\alpha}_{k}(t)\right) \dot{\alpha}_{k}(t) e_{k}(t), e_{k}(t)\right\rangle\right| \mathrm{d} t \\
& \quad \leq C\left\|\bar{e}_{k}-e_{k}\right\|_{L^{\infty}\left(0, T ; L^{2}\left(\Omega ; \mathbb{M}_{s y m}^{n \times n}\right)\right)} \int_{0}^{T}\left\|\dot{\alpha}_{k}(t)\right\|_{\infty} .
\end{aligned}
$$

Therefore, by Lemma 2.6 we get

$$
\int_{0}^{T} \delta_{k}(t) \mathrm{d} t \leq C\left(\sup _{t \in[0, T]}\left\|\bar{\alpha}_{k}-\alpha_{k}\right\|_{m, 2}+\sup _{t \in[0, T]}\left\|\bar{e}_{k}-e_{k}\right\|_{2}+\tau\right) \int_{0}^{T}\left\|\dot{\alpha}_{k}(t)\right\|_{H^{m}(\Omega)}^{2}+\left\|E \dot{w}_{k}(t)\right\|_{2}^{2} \mathrm{~d} t .
$$

In the case $\lambda=0$ we obtain, using (2.14b),

$$
\frac{\mathrm{d}}{\mathrm{d} t} \mathcal{E}\left(\alpha_{k}(t), e_{k}(t)\right) \leq-\varepsilon\left\|\dot{\alpha}_{k}(t)\right\|_{2}^{2}+\left\langle\sigma_{k}(t), \dot{e}_{k}(t)\right\rangle+\delta_{k}^{\prime}(t),
$$

with

$$
\begin{aligned}
\delta_{k}^{\prime}(t):= & C \tau\left\|\dot{\alpha}_{k}(t)\right\|_{\infty}\left\|\dot{p}_{k}(t)\right\|_{1}-\left\langle\partial D\left(\bar{\alpha}_{k}(t)\right)-\partial D\left(\alpha_{k}(t)\right), \dot{\alpha}_{k}(t)\right\rangle-\kappa\left\langle\bar{\alpha}_{k}(t)-\alpha_{k}(t), \dot{\alpha}_{k}(t)\right\rangle_{m, 2} \\
& -\frac{1}{2}\left[\left\langle\mathbb{C}^{\prime}\left(\bar{\alpha}_{k}(t)\right) \dot{\alpha}_{k}(t) \bar{e}_{k}(t), \bar{e}_{k}(t)\right\rangle-\left\langle\mathbb{C}^{\prime}\left(\alpha_{k}(t)\right) \dot{\alpha}_{k}(t) e_{k}(t), e_{k}(t)\right\rangle\right]
\end{aligned}
$$

so that

$$
\int_{0}^{T} \delta_{k}^{\prime}(t) \mathrm{d} t \leq C\left(\sup _{t \in[0, T]}\left\|\bar{\alpha}_{k}-\alpha_{k}\right\|_{m, 2}+\sup _{t \in[0, T]}\left\|\bar{e}_{k}-e_{k}\right\|_{2}+\tau\right) \int_{0}^{T}\left\|\dot{\alpha}_{k}(t)\right\|_{H^{m}(\Omega)}^{2}+\left\|E \dot{w}_{k}(t)\right\|_{2}^{2} \mathrm{~d} t .
$$

The rest of the proof is common for both cases $\lambda=0$ and $\lambda \in(0,1]$.

Now, we have that

$$
\begin{aligned}
\left\langle\sigma_{k}(t), \dot{e}_{k}(t)\right\rangle= & \left\langle\bar{\sigma}_{k}(t), \dot{e}_{k}(t)\right\rangle+\left\langle\left[\mathbb{C}\left(\alpha_{k}(t)\right)-\mathbb{C}\left(\bar{\alpha}_{k}(t)\right)\right] e_{k}(t), \dot{e}_{k}(t)\right\rangle \\
& +\left\langle\mathbb{C}\left(\bar{\alpha}_{k}(t)\right)\left(e_{k}(t)-\bar{e}_{k}(t)\right), \dot{e}_{k}(t)\right\rangle
\end{aligned}
$$

with

$$
\begin{aligned}
& \int_{0}^{T}\left|\left\langle\left[\mathbb{C}\left(\alpha_{k}(t)\right)-\mathbb{C}\left(\bar{\alpha}_{k}(t)\right)\right] e_{k}(t), \dot{e}_{k}(t)\right\rangle+\left\langle\mathbb{C}\left(\bar{\alpha}_{k}(t)\right)\left(e_{k}(t)-\bar{e}_{k}(t)\right), \dot{e}_{k}(t)\right\rangle\right| \\
& \quad \leq C \sup _{t}\left(\left\|\alpha_{k}(t)-\bar{\alpha}_{k}(t)\right\|_{\infty}+\left\|e_{k}(t)-\bar{e}_{k}(t)\right\|_{2}\right) \int_{0}^{T}\left\|\dot{e}_{k}(t)\right\|_{2} \mathrm{~d} t .
\end{aligned}
$$

Since, by definition of interpolants and Lemma 2.1, $\operatorname{div} \bar{\sigma}_{k}(t)=0$ and $\left(\dot{u}_{k}(t), \dot{e}_{k}(t), \dot{p}_{k}(t)\right) \in A\left(\dot{w}_{k}(t)\right)$ for every $t \in[0, T]$, it follows from the integration by parts formula (1.20) that

$$
\left\langle\bar{\sigma}_{k}(t), \dot{e}_{k}(t)\right\rangle=\left\langle\bar{\sigma}_{k}(t), E \dot{w}_{k}(t)\right\rangle-\left\langle\left(\bar{\sigma}_{k}(t)\right)_{D} \mid \dot{p}_{k}(t)\right\rangle .
$$

By (2.18) (recall also (1.16)), for a.e. $t \in(0, T)$

$$
\begin{aligned}
-\left\langle\left(\bar{\sigma}_{k}(t)\right)_{D} \mid \dot{p}_{k}(t)\right\rangle \leq & -\mathcal{H}\left(\alpha_{k}(t), \dot{p}_{k}(t)\right)+C \tau\left(\left\|\dot{\alpha}_{k}(t)\right\|_{\infty}^{2}+\left\|E \dot{w}_{k}(t)\right\|_{2}^{2}\right) \\
& +C_{K} \sup _{t}\left\|\alpha_{k}(t)-\bar{\alpha}_{k}(t)\right\|_{\infty}\left\|\dot{p}_{k}(t)\right\|_{1} .
\end{aligned}
$$

Gathering (3.37), (3.38), (3.39), and (3.40), it follows that

$$
\begin{aligned}
& \int_{0}^{T}\left\langle\sigma_{k}(t), \dot{e}_{k}(t)\right\rangle \mathrm{d} t \leq \int_{0}^{T}\left\langle\bar{\sigma}_{k}(t), E \dot{w}_{k}(t)\right\rangle \mathrm{d} t-\int_{0}^{T} \mathcal{H}\left(\alpha_{k}(t), \dot{p}_{k}(t)\right) \mathrm{d} t \\
& \quad+C \sup _{t}\left(\left\|\alpha_{k}(t)-\bar{\alpha}_{k}(t)\right\|_{\infty}+\left\|e_{k}(t)-\bar{e}_{k}(t)\right\|_{2}\right) \int_{0}^{T}\left\|\dot{e}_{k}(t)\right\|_{2} \mathrm{~d} t \\
& \quad+C \tau \int_{0}^{T}\left(\left\|\dot{\alpha}_{k}(t)\right\|_{\infty}^{2}+\left\|E \dot{w}_{k}(t)\right\|_{2}^{2}\right) \mathrm{d} t+C_{K} \sup _{t}\left\|\alpha_{k}(t)-\bar{\alpha}_{k}(t)\right\|_{\infty} \int_{0}^{T}\left\|\dot{p}_{k}(t)\right\|_{1} \mathrm{~d} t .
\end{aligned}
$$

Integrating (3.33) (resp. (3.35)) between 0 and $T$, by (3.34) (resp. (3.36)) and (3.41) we get that

$$
\begin{aligned}
& \mathcal{E}_{\lambda}\left(\alpha_{k}(T), e_{k}(T) ; p_{k}, T\right)+(1-\lambda) \int_{0}^{T} \mathcal{H}\left(\alpha_{k}(t), \dot{p}_{k}(t)\right) \mathrm{d} t+\varepsilon \int_{0}^{T}\left\|\dot{\alpha}_{k}(t)\right\|_{2}^{2} \mathrm{~d} t \\
& \quad \leq \mathcal{E}\left(\alpha_{0}, e_{0}\right)+\int_{0}^{T}\left\langle\bar{\sigma}_{k}(t), E \dot{w}_{k}(t)\right\rangle \mathrm{d} t+\eta_{k},
\end{aligned}
$$

with

$$
\eta_{k}:=C\left(\sup _{t \in[0, T]}\left\|\bar{\alpha}_{k}-\alpha_{k}\right\|_{m, 2}+\sup _{t \in[0, T]}\left\|\bar{e}_{k}-e_{k}\right\|_{2}+\tau\right) \int_{0}^{T}\left\|\dot{\alpha}_{k}(t)\right\|_{m, 2}^{2}+\left\|E \dot{w}_{k}(t)\right\|_{2}^{2} \mathrm{~d} t
$$


taking into account Lemma 2.6. By (3.30), (3.31), and (2.43) we can apply Lemma 3.6 obtaining that

$$
\begin{gathered}
\int_{0}^{T} \mathcal{H}\left(\alpha_{\varepsilon}(T), \dot{p}_{\varepsilon}(t)\right) \mathrm{d} t \leq \liminf _{k \rightarrow \infty} \int_{0}^{T} \mathcal{H}\left(\alpha_{k}(T), \dot{p}_{k}(t)\right) \mathrm{d} t, \\
\int_{0}^{T} \mathcal{H}\left(\alpha_{\varepsilon}(t), \dot{p}_{\varepsilon}(t)\right) \mathrm{d} t \leq \liminf _{k \rightarrow \infty} \int_{0}^{T} \mathcal{H}\left(\alpha_{k}(t), \dot{p}_{k}(t)\right) \mathrm{d} t .
\end{gathered}
$$

Since $\bar{\sigma}_{k}(t) \rightarrow \sigma_{\varepsilon}(t)$ for every $t \in[0, T]$ and $E \dot{w}_{k}(t) \rightarrow E \dot{w}(t)$ in $L^{2}(\Omega)$ for a.e. $t \in(0, T)$, by (1.27), we have that

$$
\int_{0}^{T}\left\langle\bar{\sigma}_{k}(t), E \dot{w}_{k}(t)\right\rangle \mathrm{d} t \longrightarrow \int_{0}^{T}\left\langle\sigma_{\varepsilon}(t), E \dot{w}(t)\right\rangle \mathrm{d} t \quad \text { as } k \rightarrow \infty
$$

by the Dominated Convergence Theorem.

Convergence (3.28) gives

$$
\int_{0}^{T}\left\|\dot{\alpha}_{\varepsilon}(t)\right\|_{2}^{2} \mathrm{~d} t \leq \liminf _{k \rightarrow \infty} \int_{0}^{T}\left\|\dot{\alpha}_{k}(t)\right\|_{2}^{2} \mathrm{~d} t
$$

By (3.42), (3.43), (3.44), (3.45), and the semicontinuity of $\mathcal{E}$, we get the inequality (ev4") $)_{\mathcal{E}}$.

Proof of the Kuhn-Tucker inequality (ev3) $)_{\varepsilon}$. Let us consider a function $\beta \in L^{\infty}\left(0, T ; H^{m}(\Omega)\right)$ such that $\beta(t) \in H_{-}^{m}(\Omega)$ for a.e. $t \in(0, T)$. We can say that for every $\lambda \in[0,1]$ and a.e. $t \in(0, T)$.

$$
\begin{aligned}
0 \leq & \frac{1}{2}\left\langle\mathbb{C}^{\prime}\left(\alpha_{\varepsilon}(t)\right) \beta(t) \bar{e}_{k}(t), \bar{e}_{k}(t)\right\rangle+\left\langle\partial D\left(\bar{\alpha}_{k}(t)\right), \beta(t)\right\rangle+\kappa\left\langle\bar{\alpha}_{k}(t), \beta(t)\right\rangle_{m, 2}+\varepsilon\left\langle\dot{\alpha}_{k}(t), \beta(t)\right\rangle_{2} \\
& +\lambda\left\langle\partial_{\alpha} \widehat{\mathcal{V}}_{\mathcal{H}}\left(\alpha_{\varepsilon}(t), p_{k} ; 0, t\right), \beta(t)\right\rangle+\frac{1}{2}\left\langle\left[\mathbb{C}^{\prime}\left(\bar{\alpha}_{k}(t)\right)-\mathbb{C}^{\prime}\left(\alpha_{\varepsilon}(t)\right)\right] \bar{e}_{k}(t), \bar{e}_{k}(t)\right\rangle \\
& +\lambda\left\langle\partial_{\alpha} \widehat{\mathcal{V}}_{\mathcal{H}}\left(\bar{\alpha}_{k}(t), p_{k} ; 0, t\right)-\partial_{\alpha} \widehat{\mathcal{V}}_{\mathcal{H}}\left(\alpha_{\varepsilon}(t), p_{k} ; 0, t\right), \beta(t)\right\rangle,
\end{aligned}
$$

using (2.14a) in the case $\lambda=0$ and (2.15a) when $\lambda \in(0,1]$. By (2.43), (3.31), and by choice of $\beta$, Lemma 3.6 gives

$$
-\left\langle\partial_{\alpha} \widehat{\mathcal{V}}_{\mathcal{H}}\left(\alpha_{\varepsilon}(t), p_{\varepsilon} ; 0, t\right), \beta(t)\right\rangle \leq \liminf _{k \rightarrow \infty}\left[-\left\langle\partial_{\alpha} \widehat{\mathcal{V}}_{\mathcal{H}}\left(\alpha_{\varepsilon}(t), p_{k} ; 0, t\right), \beta(t)\right\rangle\right] .
$$

for a.e. $t \in(0, T)$.

In addition, by weak lower semicontinuity of positive semidefinite quadratic forms, we get that for a.e. $t \in(0, T)$

$$
-\left\langle\mathbb{C}^{\prime}\left(\alpha_{\varepsilon}(t)\right) \beta(t) e_{\varepsilon}(t), e_{\varepsilon}(t)\right\rangle \leq \liminf _{k \rightarrow \infty}\left[-\left\langle\mathbb{C}^{\prime}\left(\alpha_{\varepsilon}(t)\right) \beta(t) \bar{e}_{k}(t), \bar{e}_{k}(t)\right\rangle\right] .
$$

By (3.47), (3.48), and Fatou's Lemma, we have that

$$
\begin{aligned}
& -\int_{0}^{T}\left[\frac{1}{2}\left\langle\mathbb{C}^{\prime}\left(\alpha_{\varepsilon}(t)\right) \beta(t) e_{\varepsilon}(t), e_{\varepsilon}(t)\right\rangle+\lambda\left\langle\partial_{\alpha} \widehat{\mathcal{V}}_{\mathcal{H}}\left(\alpha_{\varepsilon}(t), p_{\varepsilon} ; 0, t\right), \beta(t)\right\rangle\right] \mathrm{d} t \\
& \left.\quad \leq \liminf _{k \rightarrow \infty} \int_{0}^{T}-\left[\frac{1}{2} \mathbb{C}^{\prime}\left(\alpha_{\varepsilon}(t)\right) \beta(t) \bar{e}_{k}(t), \bar{e}_{k}(t)\right\rangle+\lambda\left\langle\partial_{\alpha} \widehat{\mathcal{V}}_{\mathcal{H}}\left(\alpha_{\varepsilon}(t), p_{k} ; 0, t\right), \beta(t)\right\rangle\right] \mathrm{d} t .
\end{aligned}
$$

The fact that $\alpha_{k} \rightarrow \alpha_{\varepsilon}$ in $H^{1}\left([0, T] ; L^{2}(\Omega)\right)$ implies that $\dot{\alpha}_{k} \rightarrow \dot{\alpha}_{\varepsilon}$ in $L^{2}\left([0, T] ; L^{2}(\Omega)\right)$ and then

$$
\int_{0}^{T}\left\langle\dot{\alpha}_{k}(t), \beta(t)\right\rangle \mathrm{d} t \longrightarrow \int_{0}^{T}\left\langle\dot{\alpha}_{\varepsilon}(t), \beta(t)\right\rangle \mathrm{d} t
$$

Since $\bar{\alpha}_{k}(t) \rightarrow \alpha_{\varepsilon}(t)$ weakly in $H^{m}(\Omega)$ for every $t$, it follows that

$$
\left\langle\partial D\left(\bar{\alpha}_{k}(t)\right), \beta(t)\right\rangle \longrightarrow\left\langle\partial D\left(\alpha_{\varepsilon}(t)\right), \beta(t)\right\rangle \text { and }\left\langle\bar{\alpha}_{k}(t), \beta(t)\right\rangle_{m, 2} \longrightarrow\left\langle\alpha_{\varepsilon}(t), \beta(t)\right\rangle_{m, 2}
$$

for every $t$, thus by the Dominated Convergence Theorem

$$
\int_{0}^{T}\left[\left\langle\partial D\left(\bar{\alpha}_{k}(t)\right), \beta(t)\right\rangle+\kappa\left\langle\bar{\alpha}_{k}(t), \beta(t)\right\rangle_{m, 2}\right] \mathrm{d} t \longrightarrow \int_{0}^{T}\left[\left\langle\partial D\left(\alpha_{\varepsilon}(t)\right), \beta(t)\right\rangle+\kappa\left\langle\alpha_{\varepsilon}(t), \beta(t)\right\rangle_{m, 2}\right] \mathrm{d} t .
$$

Notice now that

$$
\begin{aligned}
& \left|\left\langle\left[\mathbb{C}^{\prime}\left(\bar{\alpha}_{k}(t)\right)-\mathbb{C}^{\prime}\left(\alpha_{\varepsilon}(t)\right)\right] \beta(t) \bar{e}_{k}(t), \bar{e}_{k}(t)\right\rangle+\lambda\left\langle\partial_{\alpha} \widehat{\mathcal{V}}_{\mathcal{H}}\left(\bar{\alpha}_{k}(t), p_{k} ; 0, t\right)-\partial_{\alpha} \widehat{\mathcal{V}}_{\mathcal{H}}\left(\alpha_{\varepsilon}(t), p_{k} ; 0, t\right), \beta(t)\right\rangle\right| \\
& \quad \leq C\left\|\bar{\alpha}_{k}(t)-\alpha_{\varepsilon}(t)\right\|_{\infty}\|\beta(t)\|_{\infty},
\end{aligned}
$$

where $C$ depends on an upper bound for the $C^{1,1}$ norm of $\mathbb{C}$ and $C_{K}$ (if $\lambda \in(0,1]$ ), $\sup _{t}\left\|\bar{e}_{k}(t)\right\|_{2}$, and $\mathcal{V}_{\mathcal{H}}\left(p_{k} ; 0, t\right)$. Integrating (3.46) from 0 and $T$ and passing to the limit as $k \rightarrow \infty$, we deduce from (3.49), 
(3.50), (3.51), and (3.52) that

$$
\begin{aligned}
0 \leq \int_{0}^{T} \frac{1}{2}[ & \left\langle\mathbb{C}^{\prime}\left(\alpha_{\varepsilon}(t)\right) \beta(t) e_{\varepsilon}(t), e_{\varepsilon}(t)\right\rangle+\left\langle\partial D\left(\alpha_{\varepsilon}(t)\right), \beta(t)\right\rangle+\kappa\left\langle\alpha_{\varepsilon}(t), \beta(t)\right\rangle_{m, 2} \\
& \left.+\lambda\left\langle\partial_{\alpha} \widehat{\mathcal{V}}_{\mathcal{H}}\left(\alpha_{\varepsilon}(t), p_{\varepsilon} ; 0, t\right), \beta(t)\right\rangle\right] \mathrm{d} t+\varepsilon \int_{0}^{T}\left\langle\dot{\alpha}_{\varepsilon}(t), \beta(t)\right\rangle \mathrm{d} t .
\end{aligned}
$$

We now fix $\beta \in H_{-}^{m}(\Omega)$ and set $\beta(t):=1_{A}(t) \beta$ where $A$ is a measurable subset of $[0, T]$. Since $A$ is arbitrary, we find

$$
\begin{aligned}
& \frac{1}{2}\left\langle\mathbb{C}^{\prime}\left(\alpha_{\varepsilon}(t)\right) \beta e_{\varepsilon}(t), e_{\varepsilon}(t)\right\rangle+\left\langle\partial D\left(\alpha_{\varepsilon}(t)\right), \beta\right\rangle+\kappa\left\langle\alpha_{\varepsilon}(t), \beta\right\rangle_{m, 2} \\
& +\lambda\left\langle\partial_{\alpha} \widehat{\mathcal{V}}_{\mathcal{H}}\left(\alpha_{\varepsilon}(t), p_{\varepsilon} ; 0, t\right), \beta\right\rangle+\varepsilon\left\langle\dot{\alpha}_{\varepsilon}(t), \beta\right\rangle \geq 0,
\end{aligned}
$$

for $t \in[0, T] \backslash E_{\beta}$, where $E_{\beta}$ is a negligible set depending on $\beta$. Thanks to the separability of $H_{-}^{m}(\Omega)$, it is easily seen that the inequality holds for every $t \in[0, T] \backslash E$, where $E$ is a negligible set independent of $\beta$. Then the Kuhn-Tucker inequality (ev3) $)_{\varepsilon}$ is proved.

Remark 3.8. By (2.10) and (3.29), there exists $C$ independent of $\varepsilon$ such that $\sup _{t}\left\|\sigma_{\varepsilon}(t)\right\|_{2} \leq C$ for every $\varepsilon$ and $t \in[0, T]$. Then, the energy balance $(\mathrm{ev} 4)_{\varepsilon}$ and (1.15) imply that

$$
\int_{0}^{T}\left\|\dot{p}_{\varepsilon}(t)\right\|_{1} \mathrm{~d} t \leq C
$$

for every $\varepsilon>0, C$ being independent of $\varepsilon$.

\section{Rescaled Quasistatic Viscosity eVolutions}

In this section we study the asymptotic behavior of $\varepsilon$-approximate viscous evolutions as $\varepsilon$ tends to 0 using the rescaling technique of $[10,25,7]$. Thanks to estimates $(3.27)$ and $(3.53)$ in Theorem 3.7 and Remark 3.8, the total arclength of the graphs of the functions $t \mapsto\left(\alpha_{\varepsilon}(t), e_{\varepsilon}(t), p_{\varepsilon}(t)\right) \in H^{m}(\Omega) \times$ $L^{2}\left(\Omega ; \mathbb{M}_{s y m}^{n \times n}\right) \times M_{b}\left(\Omega \cup \partial_{D} \Omega ; \mathbb{M}_{D}^{n \times n}\right)$ is uniformly bounded in $\varepsilon$. Then the inverse functions of the arclength reparametrizations converge uniformly to a map $t^{\circ}$, up to subsequences.

Using to the "slow" time scale $s=\left(t^{\circ}\right)^{-1}(t)$ and passing to the limit as $\varepsilon \rightarrow 0$, we obtain a rescaled quasistatic viscosity evolution. In the intervals where the original time $t=t^{\circ}(s)$ increases, such an evolution behaves as a "0-approximate viscous evolution", namely conditions $(\mathrm{ev} 0)_{\varepsilon}, \ldots,(\mathrm{ev} 4)_{\varepsilon}$ hold with $\varepsilon=0$.

Definition 4.1. Let us assume (1.5), (1.7), (1.11), and let $w$ be as in (1.27). We say that a 5tuple of Lipschitz functions $\left(\alpha^{\circ}, u^{\circ}, e^{\circ}, p^{\circ}, t^{\circ}\right)$ from $[0, S]$ into $H^{m}(\Omega ;[0,1]) \times B D(\Omega) \times L^{2}\left(\Omega ; \mathbb{M}_{s y m}^{n \times n}\right) \times$ $M_{b}\left(\Omega \cup \partial_{D} \Omega ; \mathbb{M}_{D}^{n \times n}\right) \times[0, T]$ is a rescaled quasistatic viscosity evolution in the time interval $[0, S]$ with datum $w$ if, setting for every $s \in[0, S]$

$$
\begin{gathered}
\sigma^{\circ}(s):=\mathbb{C}\left(\alpha^{\circ}(s)\right) e^{\circ}(s), \quad w^{\circ}(s):=w\left(t^{\circ}(s)\right), \quad \text { and } \\
U^{\circ}:=\left\{s \in[0, S]: t^{\circ} \text { is constant in a neighbourhood of } s\right\},
\end{gathered}
$$

the following conditions are satisfied:

(ev0) irreversibility: $t^{\circ}$ is nondecreasing and surjective, and for every $x \in \Omega$

$$
[0, S] \ni s \mapsto \alpha^{\circ}(s, x) \text { is nonincreasing; }
$$

(ev1) kinematic condition and equilibrium: for every $s \in[0, S]$

$$
\left(u^{\circ}(s), e^{\circ}(s), p^{\circ}(s)\right) \in A\left(w^{\circ}(s)\right), \quad \operatorname{div} \sigma^{\circ}(s)=0 \text { in } \Omega, \quad\left[\sigma^{\circ}(s) \nu\right]=0 \text { on } \partial_{N} \Omega ;
$$

(ev2) stress constraint: for every $s \in[0, S]$

$$
\sigma^{\circ}(s) \in \mathcal{K}_{\alpha^{\circ}(s)}(\Omega)
$$

(ev3) Kuhn-Tucker inequality in $[0, S] \backslash U^{\circ}$ : for every $s \in[0, S] \backslash U^{\circ}$

$$
\left\langle\partial_{\alpha} \mathcal{E}_{\lambda}\left(\alpha^{\circ}(s), e^{\circ}(s) ; p^{\circ}, s\right), \beta\right\rangle \geq 0 \quad \text { for every } \beta \in H_{-}^{m}(\Omega) ;
$$

(ev4) energy balance: for every $s \in[0, S]$

$$
\begin{aligned}
& \mathcal{E}_{\lambda}\left(\alpha^{\circ}(s), e^{\circ}(s) ; p^{\circ}, s\right)+(1-\lambda) \int_{0}^{s} \mathcal{H}\left(\alpha^{\circ}(\tau), \dot{p}^{\circ}(\tau)\right) \mathrm{d} \tau+\int_{0}^{s}\left\|\dot{\alpha}^{\circ}(\tau)\right\|_{2} \Psi\left(\alpha^{\circ}(\tau), e^{\circ}(\tau) ; p^{\circ}, \tau\right) \mathrm{d} \tau \\
& \quad=\mathcal{E}\left(\alpha_{0}, e_{0}\right)+\int_{0}^{s}\left\langle\sigma^{\circ}(\tau), E \dot{w}^{\circ}(\tau)\right\rangle \mathrm{d} \tau,
\end{aligned}
$$

where $\Psi$ is defined in (3.9) and we use the convention $0 \cdot \infty=0$. 
Remark 4.2. By [7, Remark 4.2] the integrals in (ev4) make sense. Moreover, by definition of $\Psi$ (see also Remark 3.3 and Lemma 3.4) and (ev3) we have that

$$
\Psi\left(\alpha^{\circ}(s), e^{\circ}(s) ; p^{\circ}, s\right)=\mathrm{d}_{2}\left(\partial_{\alpha} \mathcal{E}_{\lambda}\left(\alpha^{\circ}(s), e^{\circ}(s) ; p^{\circ}, s\right), G\right)=\sup _{\beta \in F}\left\langle-\partial_{\alpha} \mathcal{E}_{\lambda}\left(\alpha^{\circ}(s), e^{\circ}(s) ; p^{\circ}, s\right), \beta\right\rangle=0
$$

for every $s \in[0, S] \backslash U^{\circ}$.

Below we give two characterizations of the notion of rescaled quasistatic viscosity evolution: the first will be employed to derive a condition of Kuhn-Tucker type for the damage variable and a weak formulation of the Prandtl-Reuss flow rule; the second will be useful in the proof of Theorem 4.4.

Proposition 4.3. Let $\left(\alpha^{\circ}, u^{\circ}, e^{\circ}, p^{\circ}, t^{\circ}\right)$ be a 5-tuple of Lipschitz functions from $[0, S]$ into $H^{m}(\Omega ;[0,1]) \times$ $B D(\Omega) \times L^{2}\left(\Omega ; \mathbb{M}_{\text {sym }}^{n \times n}\right) \times M_{b}\left(\Omega \cup \partial_{D} \Omega ; \mathbb{M}_{D}^{n \times n}\right) \times[0, T]$ satisfying $(\operatorname{ev0})-(\operatorname{ev} 3)$. Then $\left(\alpha^{\circ}, u^{\circ}, e^{\circ}, p^{\circ}, t^{\circ}\right)$ is a rescaled quasistatic viscosity evolution, i.e. it satisfies the energy balance (ev4), if and only if any of the two following conditions holds true:

(ev4') for a.e. $s \in(0, S)$ the following hold:

- generalized Kuhn-Tucker equality:

$$
\left\langle-\partial_{\alpha} \mathcal{E}_{\lambda}\left(\alpha^{\circ}(s), e^{\circ}(s) ; p^{\circ}, s\right), \dot{\alpha}^{\circ}(s)\right\rangle=\left\|\dot{\alpha}^{\circ}(s)\right\|_{2} \Psi\left(\alpha^{\circ}(s), e^{\circ}(s) ; p^{\circ}, s\right) ;
$$

- Hill's maximum plastic work principle:

$$
\mathcal{H}\left(\alpha^{\circ}(s), \dot{p}^{\circ}(s)\right)=\left\langle\left(\sigma^{\circ}(s)\right)_{D}, \dot{p}^{\circ}(s)\right\rangle \text {. }
$$

(ev4") energy inequality:

$$
\begin{aligned}
& \mathcal{E}_{\lambda}\left(\alpha^{\circ}(S), e^{\circ}(S) ; p^{\circ}, S\right)+(1-\lambda) \int_{0}^{S} \mathcal{H}\left(\alpha^{\circ}(s), \dot{p}^{\circ}(s)\right) \mathrm{d} s+\int_{0}^{S}\left\|\dot{\alpha}^{\circ}(s)\right\|_{2} \Psi\left(\alpha^{\circ}(s), e^{\circ}(s) ; p^{\circ}, s\right) \mathrm{d} s \\
& \quad \leq \mathcal{E}\left(\alpha_{0}, e_{0}\right)+\int_{0}^{S}\left\langle\sigma^{\circ}(s), E \dot{w}^{\circ}(s)\right\rangle \mathrm{d} s .
\end{aligned}
$$

Proof. Ad $(\mathbf{e v 4}) \Longleftrightarrow\left(\mathbf{e v} 4^{\prime}\right)$ : Since $\alpha^{\circ}, e^{\circ}, p^{\circ}$ are Lipschitz, the function $s \mapsto \mathcal{E}_{\lambda}\left(\alpha^{\circ}(s), e^{\circ}(s) ; p^{\circ}, s\right)$ is absolutely continuous and for a.e. $s \in(0, S)$

$$
\frac{\mathrm{d}}{\mathrm{d} s} \mathcal{E}_{\lambda}\left(\alpha^{\circ}(s), e^{\circ}(s) ; p^{\circ}, s\right)=\left\langle\partial_{\alpha} \mathcal{E}_{\lambda}\left(\alpha^{\circ}(s), e^{\circ}(s) ; p^{\circ}, s\right), \dot{\alpha}^{\circ}(s)\right\rangle+\left\langle\sigma^{\circ}(s), \dot{e}^{\circ}(s)\right\rangle+\lambda \mathcal{H}\left(\alpha^{\circ}(s), \dot{p}^{\circ}(s)\right) .
$$

Moreover, property (ev1) and [5, Lemma 5.5] give that

$$
\left(\dot{u}^{\circ}(s), \dot{e}^{\circ}(s), \dot{p}^{\circ}(s)\right) \in A\left(\dot{w}^{\circ}(s)\right) \quad \text { for a.e. } s \in(0, S),
$$

and then the integration by parts formula (1.20) implies

$$
\left\langle\left(\sigma^{\circ}(s)\right)_{D} \mid \dot{p}^{\circ}(s)\right\rangle=\left\langle\sigma^{\circ}(s), E \dot{w}^{\circ}(s)\right\rangle-\left\langle\sigma^{\circ}(s), \dot{e}^{\circ}(s)\right\rangle
$$

for a.e. $s \in(0, S)$. Then (ev4) holds if and only if

$$
\begin{aligned}
\frac{\mathrm{d}}{\mathrm{d} s} \mathcal{E}_{\lambda}\left(\alpha^{\circ}(s), e^{\circ}(s) ; p^{\circ}, s\right)= & -(1-\lambda) \mathcal{H}\left(\alpha^{\circ}(s), \dot{p}^{\circ}(s)\right)+\left\langle\sigma^{\circ}(s), E \dot{w}^{\circ}(s)\right\rangle \\
& -\left\|\dot{\alpha}^{\circ}(s)\right\|_{2} \Psi\left(\alpha^{\circ}(s), e^{\circ}(s) ; p^{\circ}, s\right),
\end{aligned}
$$

which in turn is equivalent to

$$
\begin{aligned}
& \left\langle\partial_{\alpha} \mathcal{E}_{\lambda}\left(\alpha^{\circ}(s), e^{\circ}(s) ; p^{\circ}, s\right), \dot{\alpha}^{\circ}(s)\right\rangle-\left\|\dot{\alpha}^{\circ}(s)\right\|_{2} \inf _{\beta \in F}\left\langle\partial_{\alpha} \mathcal{E}_{\lambda}\left(\alpha^{\circ}(s), e^{\circ}(s) ; p^{\circ}, s\right), \beta\right\rangle \\
& +\mathcal{H}\left(\alpha^{\circ}(s), \dot{p}^{\circ}(s)\right)-\left\langle\left(\sigma^{\circ}(s)\right)_{D} \mid \dot{p}^{\circ}(s)\right\rangle=0,
\end{aligned}
$$

see (3.9) for the definition of $\Psi$. Now, by (ev2) and (1.22), and since $\dot{p}^{\circ}(s) \in \Pi(\Omega)$ for a.e. $s$, we can say that

$$
\left\langle\left(\sigma^{\circ}(s)\right)_{D} \mid \dot{p}^{\circ}(s)\right\rangle \leq \mathcal{H}\left(\alpha^{\circ}(s), \dot{p}^{\circ}(s)\right)
$$

for a.e. $s \in(0, S)$. Then $(4.5)$ is equivalent to $\left(\mathrm{ev} 4^{\prime}\right)$.

Ad (ev4) $\Longleftrightarrow($ ev4"): It is obvious that (ev4) implies (ev4"); let us prove the converse. By (4.3), (4.4), and (4.6) we deduce that

$$
\begin{aligned}
\frac{\mathrm{d}}{\mathrm{d} s} \mathcal{E}_{\lambda}\left(\alpha^{\circ}(s), e^{\circ}(s) ; p^{\circ}, s\right) \geq & -(1-\lambda) \mathcal{H}\left(\alpha^{\circ}(s), \dot{p}^{\circ}(s)\right)+\left\|\dot{\alpha}^{\circ}(s)\right\|_{2} \inf _{\beta \in F}\left\langle\partial_{\alpha} \mathcal{E}_{\lambda}\left(\alpha^{\circ}(s), e^{\circ}(s) ; p^{\circ}, s\right), \beta\right\rangle \\
& +\left\langle\sigma^{\circ}(s), E \dot{w}^{\circ}(s)\right\rangle
\end{aligned}
$$


for a.e. $s \in(0, S)$. Integrating, we get for every $0 \leq s_{1} \leq s_{2} \leq S$ the inequality

$$
\begin{aligned}
& \mathcal{E}_{\lambda}\left(\alpha^{\circ}\left(s_{2}\right), e^{\circ}\left(s_{2}\right) ; p^{\circ}, s_{2}\right)+(1-\lambda) \int_{s_{1}}^{s_{2}} \mathcal{H}\left(\alpha^{\circ}(s), \dot{p}^{\circ}(s)\right) \mathrm{d} s+\int_{s_{1}}^{s_{2}}\left\|\dot{\alpha}^{\circ}(s)\right\|_{2} \Psi\left(\alpha^{\circ}(s), e^{\circ}(s) ; p^{\circ}, s\right) \mathrm{d} s \\
& \quad \geq \mathcal{E}_{\lambda}\left(\alpha^{\circ}\left(s_{1}\right), e^{\circ}\left(s_{1}\right) ; p^{\circ}, s_{1}\right)+\int_{s_{1}}^{s_{2}}\left\langle\sigma^{\circ}(s), E \dot{w}^{\circ}(s)\right\rangle \mathrm{d} s,
\end{aligned}
$$

which implies the energy balance (ev4) thanks to (ev4"). This concludes the proof.

The following theorem is the main result of the paper.

Theorem 4.4. Assume (1.5), (1.7), (1.11), and let $w$ and $\alpha_{0}, u_{0}, e_{0}, p_{0}$ satisfy (1.27) and (1.28) respectively. If $\lambda \in(0,1]$, assume also (1.13). Then there exist $S>0$ and a rescaled quasistatic viscosity evolution in the time interval $[0, S]$ according to Definition 4.1 such that $\left(\alpha_{0}, u_{0}, e_{0}, p_{0}, 0\right)=$ $\left(\alpha^{\circ}(0), u^{\circ}(0), e^{\circ}(0), p^{\circ}(0), t^{\circ}(0)\right)$.

Proof. The proof is divided in subsequent steps.

Viscous approximation. Let $\left\{\left(\alpha_{\varepsilon}, u_{\varepsilon}, e_{\varepsilon}, p_{\varepsilon}\right)\right\}_{\varepsilon>0}$ be a family of $\varepsilon$-approximate viscous evolutions satisfying (3.27), whose existence follows from Theorem 3.7. For every $\varepsilon>0$ and $t \in[0, T]$ let us define the function

$$
s_{\varepsilon}^{\circ}(t):=t+\int_{0}^{t}\left\|\dot{\alpha}_{\varepsilon}(s)\right\|_{m, 2} \mathrm{~d} s+\int_{0}^{t}\left\|\dot{e}_{\varepsilon}(s)\right\|_{2} \mathrm{~d} s+\int_{0}^{t}\left\|\dot{p}_{\varepsilon}(s)\right\|_{1} \mathrm{~d} s .
$$

It is easy to see that $s_{\varepsilon}^{\circ}$ is absolutely continuous, increasing, bijective on its domain, and

$$
s_{\varepsilon}^{\circ}\left(t_{2}\right)-s_{\varepsilon}^{\circ}\left(t_{1}\right) \geq t_{2}-t_{1} \quad \text { for every } 0 \leq t_{1} \leq t_{2} \leq S_{\varepsilon}:=s_{\varepsilon}^{\circ}(T) .
$$

Let $t_{\varepsilon}^{\circ}:\left[0, S_{\varepsilon}\right] \mapsto[0, T]$ be the inverse of $s_{\varepsilon}^{\circ}$. By (3.27) and (3.53), it follows that $\sup _{\varepsilon} S_{\varepsilon}<+\infty$ and then, up to a subsequence, $S_{\varepsilon} \rightarrow S$ as $\varepsilon \rightarrow 0$, with $S \geq T$, since $S_{\varepsilon}(T) \geq T$. For every $\varepsilon>0$, define the rescaled functions on $\left[0, S_{\varepsilon}\right]$ by

$$
\begin{array}{rrrl}
\alpha_{\varepsilon}^{\circ}(s):=\alpha_{\varepsilon}\left(t_{\varepsilon}^{\circ}(s)\right), & u_{\varepsilon}^{\circ}(s):=u_{\varepsilon}\left(t_{\varepsilon}^{\circ}(s)\right), & e_{\varepsilon}^{\circ}(s):=e_{\varepsilon}\left(t_{\varepsilon}^{\circ}(s)\right), \\
p_{\varepsilon}^{\circ}(s):=p_{\varepsilon}\left(t_{\varepsilon}^{\circ}(s)\right), & \sigma_{\varepsilon}^{\circ}(s):=\sigma_{\varepsilon}\left(t_{\varepsilon}^{\circ}(s)\right), & w_{\varepsilon}^{\circ}(s):=w\left(t_{\varepsilon}^{\circ}(s)\right) .
\end{array}
$$

Up to assuming that the rescaled functions and $t_{\varepsilon}^{\circ}$ take their value at $S_{\varepsilon}$ also in $\left(S_{\varepsilon}, \bar{S}\right]$, with $\bar{S}:=$ $\sup _{\varepsilon>0} S_{\varepsilon}$, we may consider them to be defined on the fixed time interval $[0, S]$.

By compactness we may assume that $t_{\varepsilon}^{\circ}$ converges weakly* in $W^{1, \infty}((0, S) ;[0, T])$ to a function $t^{\circ}$ such that $t^{\circ}(0)=0$ and

$$
0 \leq t^{\circ}\left(s_{2}\right)-t^{\circ}\left(s_{1}\right) \leq s_{2}-s_{1} \text { for every } 0 \leq s_{1} \leq s_{2} \leq S .
$$

By the uniform convergence of $t_{\varepsilon}^{\circ}$ to $t^{\circ}$ we immediately get that for every $s \in[0, S]$

$$
w_{\varepsilon}^{\circ}(s) \rightarrow w^{\circ}(s) \quad \text { in } H^{1}\left(\mathbb{R}^{n} ; \mathbb{R}^{n}\right),
$$

where we recall that $w^{\circ}(s)=w\left(t^{\circ}(s)\right)$. From the definitions of $s_{\varepsilon}^{\circ}$ and $t_{\varepsilon}^{\circ}$ we obtain that

$$
\left\|\alpha_{\varepsilon}^{\circ}\left(s_{2}\right)-\alpha_{\varepsilon}^{\circ}\left(s_{1}\right)\right\|_{m, 2}+\left\|e_{\varepsilon}^{\circ}\left(s_{2}\right)-e_{\varepsilon}^{\circ}\left(s_{1}\right)\right\|_{2}+\left\|p_{\varepsilon}^{\circ}\left(s_{2}\right)-p_{\varepsilon}^{\circ}\left(s_{1}\right)\right\|_{1} \leq s_{2}-s_{1}
$$

for every $0 \leq s_{1}<s_{2} \leq S$. Arguing as in [7, proof of (5.29)-(5.32)] and using (4.8) we see that there exist a quadruple of functions $\left(\alpha^{\circ}, u^{\circ}, e^{\circ}, p^{\circ}\right)$ from $[0, S]$ into $H^{m}(\Omega) \times B D(\Omega) \times L^{2}\left(\Omega ; \mathbb{M}_{s y m}^{n \times n}\right) \times$ $M_{b}\left(\Omega \cup \partial_{D} \Omega ; \mathbb{M}_{D}^{n \times n}\right)$, such that, up to a (not relabeled) subsequence of $\alpha_{\varepsilon}^{\circ}, u_{\varepsilon}^{\circ}, e_{\varepsilon}^{\circ}, p_{\varepsilon}^{\circ}$, it holds

$$
\begin{aligned}
& \alpha_{\varepsilon}^{\circ}\left(s_{\varepsilon}\right) \rightarrow \alpha^{\circ}(s) \text { weakly in } H^{m}(\Omega), \\
& u_{\varepsilon}^{\circ}\left(s_{\varepsilon}\right) \rightarrow u^{\circ}(s) \text { weakly* in } B D(\Omega), \\
& e_{\varepsilon}^{\circ}\left(s_{\varepsilon}\right) \rightarrow e^{\circ}(s) \text { weakly in } L^{2}\left(\Omega ; \mathbb{M}_{s y m}^{n \times n}\right), \\
& p_{\varepsilon}^{\circ}\left(s_{\varepsilon}\right) \rightarrow p^{\circ}(s) \text { weakly* in } M_{b}\left(\Omega \cup \partial_{D} \Omega ; \mathbb{M}_{D}^{n \times n}\right),
\end{aligned}
$$

for every $s \in[0, S]$ and $s_{\varepsilon} \rightarrow s$. Moreover $\left(u^{\circ}(s), e^{\circ}(s), p^{\circ}(s)\right) \in A\left(w^{\circ}(s)\right), \operatorname{div} \sigma^{\circ}(s)=0$, and

$$
\alpha_{\varepsilon}^{\circ} \rightarrow \alpha^{\circ} \text { in } C([0, S] ; C(\bar{\Omega})) \text {. }
$$

In particular (ev0) and (ev1) follow. By lower semicontinuity we obtain from (4.8) that

$$
\left\|\alpha^{\circ}\left(s_{2}\right)-\alpha^{\circ}\left(s_{1}\right)\right\|_{m, 2}+\left\|e^{\circ}\left(s_{2}\right)-e^{\circ}\left(s_{1}\right)\right\|_{2}+\left\|p^{\circ}\left(s_{2}\right)-p^{\circ}\left(s_{1}\right)\right\|_{1} \leq s_{2}-s_{1}
$$

for every $0 \leq s_{1}<s_{2} \leq S$, hence

$$
\left\|\dot{\alpha}^{\circ}(s)\right\|_{m, 2}+\left\|\dot{e}^{\circ}(s)\right\|_{2}+\left\|\dot{p}^{\circ}(s)\right\|_{1} \leq 1 \quad \text { for a.e. } s \in[0, S] .
$$

We now define

$$
\begin{array}{ll}
s_{-}^{\circ}(t):=\sup \left\{s \in[0, S]: t^{\circ}(s)<t\right\} & \text { for } t \in(0, T], \\
s_{+}^{\circ}(t):=\inf \left\{s \in[0, S]: t^{\circ}(s)>t\right\} & \text { for } t \in[0, T),
\end{array}
$$


and $s_{-}^{\circ}(0):=0, s_{+}^{\circ}(T):=S$. Then

$$
s_{-}^{\circ}(t) \leq \liminf _{\varepsilon \rightarrow 0} s_{\varepsilon}^{\circ}(t) \leq \limsup _{\varepsilon \rightarrow 0} s_{\varepsilon}^{\circ}(t) \leq s_{+}^{\circ}(t) \quad \text { and } \quad t^{\circ}\left(s_{-}^{\circ}(t)\right)=t=t^{\circ}\left(s_{+}^{\circ}(t)\right)
$$

for every $t \in[0, T]$,

for every $s \in[0, S]$, the set

$$
s_{-}^{\circ}\left(t^{\circ}(s)\right) \leq s \leq s_{+}^{\circ}\left(t^{\circ}(s)\right)
$$

is at most countable, and

$$
S^{\circ}:=\left\{t \in[0, T]: s_{-}^{\circ}(t)<s_{+}^{\circ}(t)\right\}
$$

where $U^{\circ}$ is defined in (4.1). Moreover, for every $t \in[0, T] \backslash S^{\circ}$,

$$
\begin{aligned}
& u_{\varepsilon}(t) \rightarrow u^{\circ}\left(s_{-}^{\circ}(t)\right) \text { weakly* in } B D(\Omega), \\
& e_{\varepsilon}(t) \rightarrow e^{\circ}\left(s_{-}^{\circ}(t)\right) \text { weakly in } L^{2}\left(\Omega ; \mathbb{M}_{s y m}^{n \times n}\right), \\
& p_{\varepsilon}(t) \rightarrow p^{\circ}\left(s_{-}^{\circ}(t)\right) \text { weakly }{ }^{*} \text { in } M_{b}\left(\Omega \cup \partial_{D} \Omega ; \mathbb{M}_{D}^{n \times n}\right), \\
& \alpha_{\varepsilon}(t) \rightarrow \alpha^{\circ}\left(s_{-}^{\circ}(t)\right) \text { strongly in } C(\bar{\Omega}) .
\end{aligned}
$$

These convergences will be used at the end of the proof.

From $(\mathrm{ev} 2)_{\varepsilon}$ and $(4.7)$ we have

$$
\sigma_{\varepsilon}^{\circ}(s) \in \mathcal{K}_{\alpha_{\varepsilon}^{\circ}(s)} \text { for every } s \in[0, S],
$$

thus the convexity of $K(\alpha)$ for every $\alpha \in[0,1],(1.11 \mathrm{c}$ ) and (4.9) imply (ev2). By Proposition 4.3, in order to show that $\left(\alpha^{\circ}, u^{\circ}, e^{\circ}, p^{\circ}, t^{\circ}\right)$ is a rescaled quasistatic viscosity evolution it remains to prove only (ev3) and inequality (ev4").

Proof of (ev3). Setting

$$
A^{\circ}:=\left\{s \in[0, S]: \Psi\left(\alpha^{\circ}(s), e^{\circ}(s) ; p^{\circ}, s\right)>0\right\},
$$

in order to get (ev3) it is enough to show that $A^{\circ} \subset U^{\circ}$.

Arguing as in the proof of the energy inequality (ev4") $)_{\varepsilon}$ in Theorem 3.7 and using (4.9c), (4.9d), (4.10), we see that for every $s \in[0, S]$ and $\beta \in H_{-}^{m}(\Omega)$

$$
\left\langle-\partial_{\alpha} \mathcal{E}_{\lambda}\left(\alpha^{\circ}(s), e^{\circ}(s) ; p^{\circ}, s\right), \beta\right\rangle \leq \liminf _{\varepsilon \rightarrow 0}\left\langle-\partial_{\alpha} \mathcal{E}_{\lambda}\left(\alpha_{\varepsilon}^{\circ}(s), e_{\varepsilon}^{\circ}(s) ; p_{\varepsilon}^{\circ}, s\right), \beta\right\rangle,
$$

thus

$$
\Psi\left(\alpha^{\circ}(s), e^{\circ}(s) ; p^{\circ}, s\right) \leq \liminf _{\varepsilon \rightarrow 0} \Psi\left(\alpha_{\varepsilon}^{\circ}(s), e_{\varepsilon}^{\circ}(s) ; p_{\varepsilon}^{\circ}, s\right) .
$$

Moreover, for every $\beta \in H_{-}^{m}(\Omega) s \mapsto\left\langle\partial_{\alpha} \widehat{\mathcal{V}}_{\mathcal{H}}\left(\alpha^{\circ}(s), p^{\circ} ; 0, s\right), \beta\right\rangle$ is continuous, being an integral function. Together with (4.11), this implies that $s \mapsto\left\langle-\partial_{\alpha} \mathcal{E}_{\lambda}\left(\alpha^{\circ}(s), e^{\circ}(s) ; p^{\circ}, s\right), \beta\right\rangle$ is continuous for every $\beta \in$ $H_{-}^{m}(\Omega)$, and consequently that

$$
s \mapsto \Psi\left(\alpha^{\circ}(s), e^{\circ}(s) ; p^{\circ}, s\right) \text { is lower semicontinuous . }
$$

Thus, $A^{\circ}$ is open.

We now set $D^{\circ}:=\left\{s \in(0, S): \dot{t}^{\circ}(s)=0\right\}$ and prove that

$$
\limsup _{\varepsilon \rightarrow 0} \dot{t}_{\varepsilon}^{\circ}(s)>0 \quad \text { for a.e. } s \in(0, S) \backslash D^{\circ} .
$$

Indeed, assuming the opposite, we could find a measurable set $A \subset(0, S) \backslash D^{\circ}$ with positive measure such that

$$
\lim _{\varepsilon \rightarrow 0} \dot{t}_{\varepsilon}^{\circ}(s)=0 \quad \text { for every } s \in A,
$$

$t_{\varepsilon}^{\circ}$ being nondecreasing. Since the functions $t_{\varepsilon}^{\circ}$ are 1-Lipschitz, the Dominated Convergence Theorem implies that

$$
\lim _{\varepsilon \rightarrow 0} \int_{A} \dot{t}_{\varepsilon}^{\circ}(s) \mathrm{d} s=0 .
$$

On the other hand,

$$
\lim _{\varepsilon \rightarrow 0} \int_{A} \dot{t}_{\varepsilon}^{\circ}(s) \mathrm{d} s=\int_{A} \dot{t}^{\circ}(s) \mathrm{d} s,
$$

because $t_{\varepsilon}^{\circ} \rightarrow t^{\circ}$ weakly* in $W^{1, \infty}$. But

$$
\int_{A} \dot{t}^{\circ}(s) \mathrm{d} s>0
$$

since $\dot{t}^{\circ}(s)>0$ for a.e. $s \in(0, S) \backslash D^{\circ}$. Then (4.18) is proved. 
Since $\mathcal{H}$ is 1 -homogeneous in the second variable, the reparametrization $t=t_{\varepsilon}^{\circ}(s)$ gives

$$
\int_{0}^{t_{\varepsilon}^{\circ}(S)} \mathcal{H}\left(\alpha_{\varepsilon}(t), \dot{p}_{\varepsilon}(t)\right) \mathrm{d} t=\int_{0}^{S} \mathcal{H}\left(\alpha_{\varepsilon}^{\circ}(s), \dot{p}_{\varepsilon}^{\circ}(s)\right) \mathrm{d} s .
$$

By (1.24), for every $s \in[0, S]$ and $\beta \in C(\bar{\Omega})$

$$
\left\langle\partial_{\alpha} \widehat{\mathcal{V}}_{\mathcal{H}}\left(\alpha_{\varepsilon}\left(t_{\varepsilon}^{\circ}(s)\right), p_{\varepsilon} ; 0, t_{\varepsilon}^{\circ}(s)\right), \beta\right\rangle=\left\langle\partial_{\alpha} \widehat{\mathcal{V}}_{\mathcal{H}}\left(\alpha_{\varepsilon}^{\circ}(s), p_{\varepsilon}^{\circ} ; 0, s\right), \beta\right\rangle,
$$

thus

By $(4.16)$

$$
\left\langle\partial_{\alpha} \mathcal{E}_{\lambda}\left(\alpha_{\varepsilon}\left(t_{\varepsilon}^{\circ}(s)\right), e_{\varepsilon}\left(t_{\varepsilon}^{\circ}(s)\right) ; p_{\varepsilon}, t_{\varepsilon}^{\circ}(s)\right), \beta\right\rangle=\left\langle\partial_{\alpha} \mathcal{E}_{\lambda}\left(\alpha_{\varepsilon}^{\circ}(s), e_{\varepsilon}^{\circ}(s) ; p_{\varepsilon}^{\circ}, s\right), \beta\right\rangle
$$

$$
\begin{aligned}
0 \leq \Psi\left(\alpha^{\circ}(s), e^{\circ}(s) ; p^{\circ}, s\right) & \leq \liminf _{\varepsilon \rightarrow 0} \Psi\left(\alpha_{\varepsilon}^{\circ}(s), e_{\varepsilon}^{\circ}(s) ; p_{\varepsilon}^{\circ}, s\right)=\liminf _{\varepsilon \rightarrow 0} \varepsilon\left\|\dot{\alpha}_{\varepsilon}\left(t_{\varepsilon}^{\circ}(s)\right)\right\|_{2} \\
& =\liminf _{\varepsilon \rightarrow 0} \varepsilon \frac{\left\|\dot{\alpha}_{\varepsilon}^{\circ}(s)\right\|_{2}}{\dot{t}_{\varepsilon}^{\circ}(s)}=0
\end{aligned}
$$

for a.e. $s \in(0, S) \backslash D^{\circ}$, where the first equality follows from (3.7), (3.9), and (4.21) and the last from (4.8) and (4.18). Therefore for a.e. $s \in A^{\circ}$ we have $\dot{t}^{\circ}(s)=0$. Since $A^{\circ}$ is open by (4.17), every $s \in A^{\circ}$ has an open neighborhood where $\dot{t}^{\circ}=0$; then $A^{\circ} \subset U^{\circ}$ since $t^{\circ}$ is Lipschitz and hence absolutely continuous.

Proof of the energy inequality (ev4"). Using the change of variable $t=t_{\varepsilon}^{\circ}(s)$ in the left-hand side of (3.8), we get by (4.19), (4.20), and (4.21)

$$
\begin{aligned}
& \mathcal{E}_{\lambda}\left(\alpha_{\varepsilon}^{\circ}(S), e_{\varepsilon}^{\circ}(S) ; p_{\varepsilon}^{\circ}, S\right)+(1-\lambda) \int_{0}^{S} \mathcal{H}\left(\alpha_{\varepsilon}^{\circ}(s), \dot{p}_{\varepsilon}^{\circ}(s)\right) \mathrm{d} s+\int_{0}^{S}\left\|\dot{\alpha}_{\varepsilon}^{\circ}(s)\right\|_{2} \Psi\left(\alpha_{\varepsilon}^{\circ}(s), e_{\varepsilon}^{\circ}(s) ; p_{\varepsilon}^{\circ}, s\right) \mathrm{d} s \\
& \quad=\mathcal{E}\left(\alpha_{0}, e_{0}\right)+\int_{0}^{t_{\varepsilon}^{\circ}(S)}\left\langle\sigma_{\varepsilon}(t), E \dot{w}(t)\right\rangle \mathrm{d} t .
\end{aligned}
$$

By $(4.9 \mathrm{~d}),(4.10),(4.11)$, and using Lemma 3.6 we deduce that

$$
\begin{aligned}
& \int_{0}^{S} \mathcal{H}\left(\alpha^{\circ}(s), \dot{p}^{\circ}(s)\right) \mathrm{d} s \leq \liminf _{\varepsilon \rightarrow 0} \int_{0}^{S} \mathcal{H}\left(\alpha_{\varepsilon}^{\circ}(s), \dot{p}_{\varepsilon}^{\circ}(s)\right) \mathrm{d} s \\
& \int_{0}^{S} \mathcal{H}\left(\alpha^{\circ}(S), \dot{p}^{\circ}(s)\right) \mathrm{d} s \leq \liminf _{\varepsilon \rightarrow 0} \int_{0}^{S} \mathcal{H}\left(\alpha_{\varepsilon}^{\circ}(S), \dot{p}_{\varepsilon}^{\circ}(s)\right) \mathrm{d} s .
\end{aligned}
$$

Let us now prove that

$$
\int_{A^{\circ}}\left\|\dot{\alpha}^{\circ}(s)\right\|_{2} \Psi\left(\alpha^{\circ}(s), e^{\circ}(s) ; p^{\circ}, s\right) \mathrm{d} s \leq \liminf _{\varepsilon \rightarrow 0} \int_{A^{\circ}}\left\|\dot{\alpha}_{\varepsilon}^{\circ}(s)\right\|_{2} \Psi\left(\alpha_{\varepsilon}^{\circ}(s), e_{\varepsilon}^{\circ}(s) ; p_{\varepsilon}^{\circ}, s\right) \mathrm{d} s .
$$

For every compact set $C \subset A^{\circ}$ and every continuous function $\psi: C \rightarrow[0,+\infty)$ such that

$$
\Psi\left(\alpha^{\circ}(s), e^{\circ}(s) ; p^{\circ}, s\right)>\psi(s) \text { for every } s \in C,
$$

by the compactness of $C$ and (4.16), for $\varepsilon$ sufficiently small we get

$$
\Psi\left(\alpha_{\varepsilon}^{\circ}(s), e_{\varepsilon}^{\circ}(s) ; p_{\varepsilon}^{\circ}, s\right)>\psi(s) \text { for every } s \in C .
$$

We now claim that

$$
\int_{C}\left\|\dot{\alpha}^{\circ}(s)\right\|_{2} \psi(s) \mathrm{d} s \leq \liminf _{\varepsilon \rightarrow 0} \int_{C}\left\|\dot{\alpha}_{\varepsilon}^{\circ}(s)\right\|_{2} \psi(s) \mathrm{d} s
$$

for every compact $C \subset A^{\circ}$ and every continuous function $\psi: C \rightarrow[0,+\infty)$. This can be proved as in $[7$, Lemma 6.4] using (4.8) and (4.9a) and noticing that for every $\varphi \in C_{c}(\Omega)$ with $\|\varphi\|_{2}=1$ the functions $s \mapsto\left\langle\varphi, \dot{\alpha}_{\varepsilon}^{\circ}(s)\right\rangle$ are equi-Lipschitz on $[0, S]$ and converge to $s \mapsto\left\langle\varphi, \dot{\alpha}^{\circ}(s)\right\rangle$ for every $s$. By $(4.17)$ and a standard approximation argument, (4.24) follows.

Let us now consider the left-hand side of (4.22): by (4.9), (4.23), and (4.24) we have

$$
\begin{aligned}
\mathcal{E}_{\lambda}\left(\alpha^{\circ}(S), e^{\circ}(S) ; p^{\circ}, S\right)+(1-\lambda) \int_{0}^{S} \mathcal{H}\left(\alpha^{\circ}(s), \dot{p}^{\circ}(s)\right) \mathrm{d} s+\int_{0}^{S}\left\|\dot{\alpha}^{\circ}(s)\right\|_{2} \Psi\left(\alpha^{\circ}(s), e^{\circ}(s) ; p^{\circ}, s\right) \mathrm{d} s \\
\leq \liminf _{\varepsilon \rightarrow 0}\left[\mathcal{E}_{\lambda}\left(\alpha_{\varepsilon}^{\circ}(S), e_{\varepsilon}^{\circ}(S) ; p_{\varepsilon}^{\circ}, S\right)+(1-\lambda) \int_{0}^{S} \mathcal{H}\left(\alpha_{\varepsilon}^{\circ}(s), \dot{p}_{\varepsilon}^{\circ}(s)\right) \mathrm{d} s\right. \\
\left.+\int_{0}^{S}\left\|\dot{\alpha}_{\varepsilon}^{\circ}(s)\right\|_{2} \Psi\left(\alpha_{\varepsilon}^{\circ}(s), e_{\varepsilon}^{\circ}(s) ; p_{\varepsilon}^{\circ}, s\right) \mathrm{d} s\right] .
\end{aligned}
$$

As for the right-hand side, by (4.14) and the Dominated Convergence Theorem,

$$
\int_{0}^{T}\left\langle\sigma^{\circ}\left(s_{-}^{\circ}(t)\right), E \dot{w}(t)\right\rangle \mathrm{d} t=\lim _{\varepsilon \rightarrow 0} \int_{0}^{t_{\varepsilon}^{\circ}(S)}\left\langle\sigma_{\varepsilon}(t), E \dot{w}(t)\right\rangle \mathrm{d} t .
$$


Since $t^{\circ}$ is nondecreasing and Lipschitz, by (1.27) the function $w^{\circ}$ is absolutely continuous and

$$
E \dot{w}^{\circ}(s)=E \dot{w}\left(t^{\circ}(s)\right) \dot{t}^{\circ}(s) \text { for a.e. } s \in[0, S] .
$$

Hence

$$
\int_{0}^{T}\left\langle\sigma^{\circ}\left(s_{-}^{\circ}(t)\right), E \dot{w}(t)\right\rangle \mathrm{d} t=\int_{0}^{S}\left\langle\sigma^{\circ}\left(s_{-}^{\circ}\left(t^{\circ}(s)\right)\right), E \dot{w}\left(t^{\circ}(s)\right) \dot{t}^{\circ}(s)\right\rangle \mathrm{d} s=\int_{0}^{S}\left\langle\sigma^{\circ}(s), E \dot{w}^{\circ}(s)\right\rangle \mathrm{d} s .
$$

The last equality holds since $\dot{t}^{\circ}(s)=0$ for a.e. $s \in U^{\circ}$ and $s_{-}^{\circ}\left(t^{\circ}(s)\right)=s$ for a.e. $s \in[0, S] \backslash U^{\circ}$. (The only exceptions are the points of the form $s=s_{+}^{\circ}(t)$ for $t \in S^{\circ}$.) From (4.22), (4.25), (4.26), and (4.27) we get finally the energy inequality (ev4"). Thus the proof is completed.

Remark 4.5. From (4.2a) and (4.15) we immediately get the classical Kuhn-Tucker conditions in $[0, S] \backslash A^{\circ}:$

- For every $s \in[0, S] \backslash A^{\circ}$

$$
\left\langle\partial_{\alpha} \mathcal{E}_{\lambda}\left(\alpha^{\circ}(s), e^{\circ}(s) ; p^{\circ}, s\right), \beta\right\rangle \geq 0 \text { for every } \beta \in H_{-}^{m}(\Omega) .
$$

- For a.e. $s \in[0, S] \backslash A^{\circ}$

$$
\left\langle\partial_{\alpha} \mathcal{E}_{\lambda}\left(\alpha^{\circ}(s), e^{\circ}(s) ; p^{\circ}, s\right), \dot{\alpha}^{\circ}(s)\right\rangle=0 .
$$

\section{Properties of Rescaled Quasistatic Viscosity evolutions}

In the following we highlight some properties of rescaled viscosity evolutions, whose existence has been proved in Section 4 by time rescaling $[10,25,7]$.

In the first part of this section we study what happens when the original time scale $t=t^{\circ}(s)$ is constant, i.e., in the jumping regime. In Lemma 5.1 we observe that if the damage variable is constant in a subinterval of $U^{\circ}$, then also the other variables are constant. On the other hand, if $\dot{\alpha}^{\circ}>0$ in an interval then, up to a further time rescaling, the evolution is governed formally by $(\mathrm{ev} 0)_{\varepsilon}, \ldots,(\mathrm{ev} 4)_{\varepsilon}$ with $\varepsilon=1$ (see Proposition 5.3 and Remark 5.4).

Moreover, exploiting the results $[5,12,33]$ in Proposition 5.5 we recover a weak formulation of the Prandtl-Reuss flow rule, in the presence of damage. Together with conditions (ev1) and (ev2), this flow rule characterizes the perfect plasticity.

Finally, following [8], we come back to the original time variable $t$ and correspondingly we define the notion of quasistatic viscosity evolution. Such an evolution satisfies an energy balance with terms depending only on $t$; the energy dissipated during the jumping regime is thus concentrated on the jump instants. The state after a jump is known through the slow time scale description, which allows then evaluating the dissipation.

Henceforth we assume that $\left(\alpha^{\circ}, u^{\circ}, e^{\circ}, p^{\circ}, t^{\circ}\right)$ is a rescaled viscosity evolution in the time interval $[0, S]$ with datum $w$, and we use the notation of Section 4 .

Lemma 5.1. If $\dot{\alpha}^{\circ}(s)=0$ in $\Omega$ for every $s$ in an interval $\left(s_{1}, s_{2}\right) \subset U^{\circ}$, then

$$
u^{\circ}(s)=u^{\circ}\left(s_{1}\right), \quad e^{\circ}(s)=e^{\circ}\left(s_{1}\right), \quad p^{\circ}(s)=p^{\circ}\left(s_{1}\right), \quad t^{\circ}(s)=t^{\circ}\left(s_{1}\right) \quad \text { for every } s \in\left(s_{1}, s_{2}\right) .
$$

In other words, the evolution is trivial in $\left(s_{1}, s_{2}\right)$. Moreover, it cannot happen that $\left(s_{1}, s_{2}\right)$ is a connected component of the set $A^{\circ}$ defined in (4.15).

Proof. Let $\left(s_{1}, s_{2}\right) \subset U^{\circ}$ be such that $\dot{\alpha}^{\circ}(s)=0$ in $\Omega$ for every $s \in\left(s_{1}, s_{2}\right)$; by definition of $U^{\circ}$ we have that

and by assumption

$$
t^{\circ}(s)=t^{\circ}\left(s_{1}\right), \quad w^{\circ}(s)=w^{\circ}\left(s_{1}\right) \quad \text { for every } s \in\left(s_{1}, s_{2}\right),
$$

$$
\alpha^{\circ}(s)=\alpha^{\circ}\left(s_{1}\right) \quad \text { for every } s \in\left(s_{1}, s_{2}\right)
$$

in the interval $\left(s_{1}, s_{2}\right)$. By [33, Theorem 3.10], (ev1) and (ev2) are equivalent to the fact that the triple $\left(u^{\circ}(s), e^{\circ}(s), p^{\circ}(s)\right)$ solves the minimum problem

$$
\min _{(u, e, p) \in A\left(w^{\circ}\left(s_{1}\right)\right)}\left\{\mathcal{Q}\left(\alpha^{\circ}\left(s_{1}\right), e\right)+\mathcal{H}\left(\alpha^{\circ}\left(s_{1}\right), p-p^{\circ}(s)\right)\right\}
$$

for every $s \in\left(s_{1}, s_{2}\right)$. Moreover, in view of (5.1) and (5.2), we can write the energy balance in the time interval $\left(s_{1}, s_{2}\right)$ as

$$
\mathcal{E}\left(\alpha^{\circ}\left(s_{1}\right), e^{\circ}\left(s_{2}\right)\right)+\int_{s_{1}}^{s_{2}} \mathcal{H}\left(\alpha^{\circ}\left(s_{1}\right), \dot{p}^{\circ}(\tau)\right) \mathrm{d} \tau=\mathcal{E}\left(\alpha_{0}, e_{0}\right) .
$$

Thus $\left(u^{\circ}, e^{\circ}, p^{\circ}\right)$ is a quasistatic evolution in perfect plasticity (for heterogeneous materials) according to [33, Definition 3.13] with $\mathbb{C}=\mathbb{C}\left(\alpha^{\circ}\left(s_{1}\right)\right), K=K\left(\alpha^{\circ}\left(s_{1}\right)\right)$ and constant external loading in $\left(s_{1}, s_{2}\right)$. Then by $[33$, Theorem 3.14$]$ we deduce

$$
u^{\circ}(s)=u^{\circ}\left(s_{1}\right), \quad e^{\circ}(s)=e^{\circ}\left(s_{1}\right), \quad p^{\circ}(s)=p^{\circ}\left(s_{1}\right) \quad \text { for every } s \in\left(s_{1}, s_{2}\right) .
$$


In order to prove the final statement, assume that $\dot{\alpha}^{\circ}(s)=0$ in $\Omega$ for every $s$ in a connected component $\left(s_{1}, s_{2}\right)$ of $A^{\circ}$. This implies $\partial_{\alpha} \mathcal{E}_{\lambda}\left(\alpha^{\circ}(s), e^{\circ}(s) ; p^{\circ}, s\right)=\partial_{\alpha} \mathcal{E}_{\lambda}\left(\alpha^{\circ}\left(s_{1}\right), e^{\circ}\left(s_{1}\right) ; p^{\circ}, s_{1}\right)$ for every $s \in\left[s_{1}, s_{2}\right]$, which is impossible by definition of $A^{\circ}$ : indeed, $\Psi\left(\alpha^{\circ}\left(s_{i}\right), e^{\circ}\left(s_{i}\right) ; p^{\circ}, s_{i}\right)=0$ for $i=1,2$ and $\Psi\left(\alpha^{\circ}(s), e^{\circ}(s) ; p^{\circ}, s\right)>0$ for $s \in\left(s_{1}, s_{2}\right)$.

We now show a variational inequality describing the jumping regime and further reparametrize it.

Proposition 5.2. For a.e. $s \in(0, S)$

$$
\left\|\dot{\alpha}^{\circ}(s)\right\|_{2}\left\langle\partial_{\alpha} \mathcal{E}_{\lambda}\left(\alpha^{\circ}(s), e^{\circ}(s) ; p^{\circ}, s\right), \beta-\dot{\alpha}^{\circ}(s)\right\rangle+\Psi\left(\alpha^{\circ}(s), e^{\circ}(s) ; p^{\circ}, s\right)\left\langle\dot{\alpha}^{\circ}(s), \beta-\dot{\alpha}^{\circ}(s)\right\rangle_{2} \geq 0
$$

for every $\beta \in H_{-}^{m}(\Omega)$.

In particular, if $\dot{\alpha}^{\circ}(s) \leq-C<0$ in $\Omega$, then

$$
\left\|\dot{\alpha}^{\circ}(s)\right\|_{2}\left\langle-\partial_{\alpha} \mathcal{E}_{\lambda}\left(\alpha^{\circ}(s), e^{\circ}(s) ; p^{\circ}, s\right), \beta\right\rangle=\Psi\left(\alpha^{\circ}(s), e^{\circ}(s) ; p^{\circ}, s\right)\left\langle\dot{\alpha}^{\circ}(s), \beta\right\rangle_{2}
$$

for every $\beta \in H^{m}(\Omega)$.

Proof. In this proof it is convenient to use the characterization (3.12) of $\Psi$ in terms of $\mathrm{d}_{2}$. Let us consider the nontrivial case when $\dot{\alpha}^{\circ}(s)$ is not identically zero. Assume that $g \in L^{2}(\Omega)$ realizes the distance $\mathrm{d}_{2}\left(\partial_{\alpha} \mathcal{E}_{\lambda}\left(\alpha^{\circ}(s), e^{\circ}(s) ; p^{\circ}, s\right), G\right)$, i.e., $g+\partial_{\alpha} \mathcal{E}_{\lambda}\left(\alpha^{\circ}(s), e^{\circ}(s) ; p^{\circ}, s\right) \in G$ and

$$
\|g\|_{2}=\mathrm{d}_{2}\left(\partial_{\alpha} \mathcal{E}_{\lambda}\left(\alpha^{\circ}(s), e^{\circ}(s) ; p^{\circ}, s\right), G\right)=\Psi\left(\alpha^{\circ}(s), e^{\circ}(s) ; p^{\circ}, s\right) .
$$

By $(4.2 \mathrm{a})$ we get

$$
\|g\|_{2}\left\|\dot{\alpha}^{\circ}(s)\right\|_{2}=\left\langle-\partial_{\alpha} \mathcal{E}_{\lambda}\left(\alpha^{\circ}(s), e^{\circ}(s) ; p^{\circ}, s\right), \dot{\alpha}^{\circ}(s)\right\rangle \leq \int_{\Omega} g \dot{\alpha}^{\circ}(s) \mathrm{d} x \leq\|g\|_{2}\left\|\dot{\alpha}^{\circ}(s)\right\|_{2},
$$

where the first inequality above follows from (3.11) and the fact that $\dot{\alpha}^{\circ}(s) \in H_{-}^{m}(\Omega)$. Hence, by the Cauchy inequality $g$ is proportional to $\dot{\alpha}^{\circ}(s)$, and so

$$
g=\Psi\left(\alpha^{\circ}(s), e^{\circ}(s) ; p^{\circ}, s\right) \frac{\dot{\alpha}^{\circ}(s)}{\left\|\dot{\alpha}^{\circ}(s)\right\|_{2}} .
$$

Therefore (5.3) follows from (3.11) and (4.2a). The last assertion follows by substituting $\beta$ with $\delta \beta+\dot{\alpha}^{\circ}(s)$ in (5.3) for suitable $\delta>0$.

Proposition 5.3. Let $\left(s_{1}, s_{2}\right)$ be an interval in $A^{\circ}$ (defined in (4.15)) containing no subintervals where $\left\|\dot{\alpha}^{\circ}(s)\right\|_{2}=0$ for a.e. s. Setting

$$
\varrho(s):=\Psi\left(\alpha^{\circ}(s), e^{\circ}(s) ; p^{\circ}, s\right)
$$

and

$$
r^{\sharp}(s):=\int_{\frac{s_{1}+s_{2}}{2}}^{s} \frac{\left\|\dot{\alpha}^{\circ}(\sigma)\right\|_{2}}{\varrho(\sigma)} \mathrm{d} \sigma \quad \text { for } s \in\left(s_{1}, s_{2}\right),
$$

it turns out that $r^{\sharp}$ is locally Lipschitz and strictly monotone, and we call $s^{\sharp}$ its inverse function. Then

$$
\alpha^{\sharp}(r):=\alpha^{\circ}\left(s^{\sharp}(r)\right) \quad \text { for } r \in r^{\sharp}\left(\left(s_{1}, s_{2}\right)\right)
$$

has bounded variation and is continuous into $H^{m}(\Omega)$, and

$$
\left\|\dot{\alpha}^{\circ}\left(s^{\sharp}(r)\right)\right\|_{2}^{2}\left[\left\langle\partial_{\alpha} \mathcal{E}_{\lambda}\left(\alpha^{\sharp}(r), e^{\sharp}(r) ; p^{\sharp}, r\right), \beta-\dot{\alpha}^{\sharp}(r)\right\rangle+\left\langle\dot{\alpha}^{\sharp}(r), \beta-\dot{\alpha}^{\sharp}(r)\right\rangle_{2}\right] \geq 0
$$

for a.e. $r \in r^{\sharp}\left(\left(s_{1}, s_{2}\right)\right)$.

Proof. By (4.15), (4.17) and (5.4) it follows that for every compact set $K \subset A^{\circ}$ there exists $\delta_{K}>0$ such that $\varrho(s) \geq \delta_{K}$ for $s \in K$. Thus $r^{\sharp}$ is locally Lipschitz on $\left(s_{1}, s_{2}\right)$ and in particular $\mathcal{L}^{n}\left(r^{\sharp}(E)\right)=0$ for every $E \subset\left(s_{1}, s_{2}\right)$ such that $\mathcal{L}^{n}(E)=0$. Moreover $r^{\sharp}$ is strictly increasing, because by assumption every subinterval in $\left(s_{1}, s_{2}\right)$ has a subset of positive measure where $\left\|\dot{\alpha}^{\circ}(s)\right\|_{2}>0$. This implies that $s^{\sharp}$ is continuous and strictly increasing, and $\alpha^{\sharp}$ is continuous and has bounded variation, $\alpha^{\circ}$ being Lipschitz.

Therefore, using the change of variables $s=s^{\sharp}(r)$ in (5.3) and the analogous of (4.21), we obtain that for a.e. $r \in\left(r_{1}, r_{2}\right):=r^{\sharp}\left(\left(s_{1}, s_{2}\right)\right)$

$$
\left\|\dot{\alpha}^{\circ}\left(s^{\sharp}(r)\right)\right\|_{2}\left\langle\partial_{\alpha} \mathcal{E}_{\lambda}\left(\alpha^{\sharp}(r), e^{\sharp}(r) ; p^{\sharp}, r\right), \beta-\dot{\alpha}^{\circ}\left(s^{\sharp}(r)\right)\right\rangle+\varrho\left(s^{\sharp}(r)\right)\left\langle\dot{\alpha}^{\circ}\left(s^{\sharp}(r)\right), \beta-\dot{\alpha}^{\circ}\left(s^{\sharp}(r)\right)\right\rangle_{2} \geq 0
$$

for every $\beta \in H_{-}^{m}(\Omega)$. Since $\alpha^{\sharp}$ has bounded variation in $H^{m}(\Omega)$, it is $H^{m}(\Omega)$-weakly differentiable at a.e. $r \in\left(r_{1}, r_{2}\right)$, and the chain rule

$$
\dot{\alpha}^{\sharp}(r)=\dot{\alpha}^{\circ}\left(s^{\sharp}(r)\right) \dot{s}^{\sharp}(r)=\dot{\alpha}^{\circ}\left(s^{\sharp}(r)\right) \frac{\varrho\left(s^{\sharp}(r)\right)}{\left\|\dot{\alpha}^{\circ}\left(s^{\sharp}(r)\right)\right\|_{2}} \quad \text { a.e. in } \Omega
$$

holds for a.e. $r$ such that $\left\|\dot{\alpha}^{\circ}\left(s^{\sharp}(r)\right)\right\|_{2}>0$. Thus for a.e. $r \in\left(r_{1}, r_{2}\right)$

$$
\left\|\dot{\alpha}^{\circ}\left(s^{\sharp}(r)\right)\right\|_{2} \dot{\alpha}^{\sharp}(r)=\dot{\alpha}^{\circ}\left(s^{\sharp}(r)\right) \varrho\left(s^{\sharp}(r)\right) \quad \text { a.e. in } \Omega \text {. }
$$


By (5.7), the inequality (5.6) reads as

$$
\left\|\dot{\alpha}^{\circ}\left(s^{\sharp}(r)\right)\right\|_{2}\left[\left\langle\partial_{\alpha} \mathcal{E}_{\lambda}\left(\alpha^{\sharp}(r), e^{\sharp}(r) ; p^{\sharp}, r\right), \beta-\dot{\alpha}^{\circ}\left(s^{\sharp}(r)\right)\right\rangle+\left\langle\dot{\alpha}^{\sharp}(r), \beta-\dot{\alpha}^{\circ}\left(s^{\sharp}(r)\right)\right\rangle_{2}\right] \geq 0
$$

for every $\beta \in H_{-}^{m}(\Omega)$; so by using again (5.7) we get (5.5), since $\varrho\left(s^{\sharp}(r)\right)>0$ for a.e. $r \in\left(r_{1}, r_{2}\right)$. This concludes the proof.

Remark 5.4. In addition to the hypoteses above, let us assume that $\left\|\dot{\alpha}^{\circ}(s)\right\|_{2}>0$ for every $s \in\left(s_{1}, s_{2}\right)$ and that for every $K$ compact set in $\left(s_{1}, s_{2}\right)$ there exists $\delta_{K}>0$ such that $\left\|\dot{\alpha}^{\circ}(s)\right\|_{2} \geq \delta_{K}$ for $s \in K$. Then $r^{\sharp}$ is locally bi-Lipschitz, $\alpha^{\sharp}$ is locally Lipschitz, and

$$
\left\langle\partial_{\alpha} \mathcal{E}_{\lambda}\left(\alpha^{\sharp}(r), e^{\sharp}(r) ; p^{\sharp}, r\right), \beta-\dot{\alpha}^{\sharp}(r)\right\rangle+\left\langle\dot{\alpha}^{\sharp}(r), \beta-\dot{\alpha}^{\sharp}(r)\right\rangle_{2} \geq 0 \quad \text { for a.e. } r \in r^{\sharp}\left(\left(s_{1}, s_{2}\right)\right) .
$$

In particular, this variational inequality is equivalent to

$$
\left\{\begin{array}{l}
\left\langle\partial_{\alpha} \mathcal{E}_{\lambda}\left(\alpha^{\sharp}(r), e^{\sharp}(r) ; p^{\sharp}, r\right), \beta\right\rangle+\left\langle\dot{\alpha}^{\sharp}(r), \beta\right\rangle_{2} \geq 0 \quad \text { for a.e. } r \in r^{\sharp}\left(\left(s_{1}, s_{2}\right)\right), \\
\left.\left\langle\partial_{\alpha} \mathcal{E}_{\lambda}\left(\alpha^{\sharp}(r), e^{\sharp}(r) ; p^{\sharp}, r\right), \dot{\alpha}^{\sharp}(r)\right\rangle+\| \dot{\alpha}^{\sharp}(r)\right\rangle \|_{2}^{2}=0 .
\end{array}\right.
$$

Thus, in those intervals of $A^{\circ},\left(\alpha^{\sharp}, u^{\sharp}, e^{\sharp}, p^{\sharp}, t^{\sharp}\right):=\left(\alpha^{\circ}, u^{\circ}, e^{\circ}, p^{\circ}, t^{\circ}\right) \circ s^{\sharp}$ is a 1-approximate viscous evolution, in the sense that the evolution satisfies the same properties $(\mathrm{ev} 1)_{\varepsilon}-\left(\mathrm{ev} 4^{\prime}\right)_{\varepsilon}$ of an $\varepsilon$-approximate viscous evolution, with $\varepsilon=1$. In particular, (5.8) is the analogous of the Kuhn-Tucker conditions (ev3) $\varepsilon$ and (3.2).

We now prove a weak formulation of the Prandtl-Reuss flow rule: together with conditions (ev1) and (ev2) in Definition 4.1, this corresponds to the formulation of quasistatic evolution for perfect plasticity in the presence of damage.

Proposition 5.5 (Maximum plastic work principle and flow rule). From (4.2b), (ev2), and (1.21) we easily deduce the maximum plastic work principle:

$$
H\left(\alpha^{\circ}(s), \frac{\mathrm{d} \dot{p}^{\circ}(s)}{\mathrm{d}\left|\dot{p}^{\circ}(s)\right|}\right)\left|\dot{p}^{\circ}(s)\right|=\left[\left(\sigma^{\circ}(s)\right)_{D}: \dot{p}^{\circ}(s)\right] \quad \text { as measures on } \Omega \cup \partial_{D} \Omega \text {, }
$$

for a.e. $s \in(0, S)$, where the measure denoted by square brackets has been introduced in (1.19). Moreover, defining $\mu(s):=\mathcal{L}^{n}+\left|\dot{p}^{\circ}(s)\right|$ for every $s \in[0, S]$, there exists $\widehat{\sigma}_{D}^{\circ}(s) \in L_{\mu(s)}^{\infty}\left(\Omega \cup \partial_{D} \Omega ; \mathbb{M}_{D}^{n \times n}\right)$ for a.e. $s \in(0, S)$ such that

$$
\begin{gathered}
\widehat{\sigma}_{D}^{\circ}(s)=\sigma_{D}^{\circ}(s) \quad \mathcal{L}^{n}-\text { a.e. on } \Omega, \\
{\left[\sigma_{D}^{\circ}(s): \dot{p}^{\circ}(s)\right]=\left(\widehat{\sigma}_{D}^{\circ}(s): \frac{\mathrm{d} \dot{p}^{\circ}(s)}{\mathrm{d}\left|\dot{p}^{\circ}(s)\right|}\right)\left|\dot{p}^{\circ}(s)\right| \quad \text { on } \Omega \cup \partial_{D} \Omega,} \\
\frac{\mathrm{d} \dot{p}^{\circ}(s)}{\mathrm{d}\left|\dot{p}^{\circ}(s)\right|}(x) \in N_{K\left(\alpha^{\circ}(s, x)\right)}\left(\widehat{\sigma}_{D}^{\circ}(s, x)\right) \quad \text { for }\left|\dot{p}^{\circ}(s)\right|-\text { a.e. } x \in \Omega \cup \partial_{D} \Omega,
\end{gathered}
$$

where $\widehat{\sigma}_{D}^{\circ}(s, x)$ denotes the value of $\widehat{\sigma}_{D}^{\circ}(s)$ at the point $x$ and $N_{K\left(\alpha^{\circ}(s, x)\right)}\left(\sigma_{D}^{\circ}(s, x)\right)$ is the normal cone to the closed convex set $K\left(\alpha^{\circ}(s, x)\right)$ at $\sigma_{D}^{\circ}(s, x)$.

Proof. It is enough to repeat the same construction of the precise representative of the stress as in [5, Theorem 6.4], using [33, Lemma 3.16]. To this end, notice that in [12, Theorem 6.2] it is proved that the density of the $\mathcal{L}^{n}$-absolutely continuous part of $\left[\sigma_{D}: p\right]$ is $\sigma_{D}: p_{a}$, where $p_{a}$ is the density of the $\mathcal{L}^{n}$-absolutely continuous part of a plastic strain $p$ and $\sigma$ is an elastic stress, and that [33, Lemma 3.16] does not use the regularity of $\Omega$.

From now on we study the evolutions in terms of the original variable $t$.

Definition 5.6. Let us assume (1.5), (1.7), (1.9), (1.11), and (1.27) for a given $w$. We say that $(\alpha, u, e, p)$ is a quasistatic viscosity evolution with datum $w$ if there exists a rescaled viscosity evolution $\left(\alpha^{\circ}, u^{\circ}, e^{\circ}, p^{\circ}, t^{\circ}\right)$ with the same datum such that $t^{\circ}:[0, S] \rightarrow[0, T]$ and for every $t \in[0, T]$

$$
\alpha(t)=\alpha^{\circ}\left(s_{-}^{\circ}(t)\right), \quad u(t)=u^{\circ}\left(s_{-}^{\circ}(t)\right), \quad e(t)=e^{\circ}\left(s_{-}^{\circ}(t)\right), \quad p(t)=p^{\circ}\left(s_{-}^{\circ}(t)\right),
$$

where we recall that $s_{-}^{\circ}(t):=\sup \left\{s \in[0, S]: t^{\circ}(s)<t\right\}$. Moreover, we denote

$$
\sigma(t):=\sigma^{\circ}\left(s_{-}^{\circ}(t)\right) .
$$

By continuity with respect to time of rescaled viscosity evolutions and by left continuity of $s_{-}^{\circ}$, the functions introduced above are left-continuous in the norm topologies of their target spaces. Since

$$
\lim _{h \rightarrow 0} s_{-}^{\circ}(t+h)=s_{+}^{\circ}(t)
$$

for every $t \in[0, T]$, the right limits $\alpha\left(t^{+}\right), u\left(t^{+}\right), e\left(t^{+}\right)$, and $p\left(t^{+}\right)$in their norm topologies satisfy

$$
\alpha\left(t^{+}\right)=\alpha^{\circ}\left(s_{+}^{\circ}(t)\right), \quad u\left(t^{+}\right)=u^{\circ}\left(s_{+}^{\circ}(t)\right), \quad e\left(t^{+}\right)=e^{\circ}\left(s_{+}^{\circ}(t)\right), \quad p\left(t^{+}\right)=p^{\circ}\left(s_{+}^{\circ}(t)\right) .
$$


Notice that $p:[0, T] \rightarrow M_{b}\left(\Omega \cup \partial_{D} \Omega ; \mathbb{M}_{D}^{n \times n}\right)$ has bounded variation, since $p^{\circ}$ is Lipschitz and $s_{-}^{\circ}$ is nondecreasing. Then we define $\mu$ as the unique Radon measure on $[0, T]$ such that

$$
\mu([0, t])=\mathcal{V}(p ; 0, t),
$$

for every continuity point $t$ of $t \mapsto \mathcal{V}(p ; 0, t)$, with $\mathcal{V}(p ; 0, t)$ the total variation of $p$ on $[0, T]$ introduced in (1.23). By the continuity properties of $p$, we have that $\mu(\{t\})=0$ for every $t \notin S^{\circ}$ (recall (4.12)), and then the diffuse part $\mu_{d}$ of $\mu$ satisfies

$$
\mu_{d}=\mu-\sum_{\tau \in S^{\circ}} \mu(\{t\}) \delta_{\tau}
$$

where $\delta_{\tau}$ is the unit mass at $\tau$.

By [8, Theorem 7.1], there is a unique (up to $\mu$-equivalence) function $\nu_{p}:[0, T] \rightarrow M_{b}\left(\Omega \cup \partial_{D} \Omega ; \mathbb{M}_{D}^{n \times n}\right)$ such that for every $\varphi \in C_{0}\left(\Omega \cup \partial_{D} \Omega ; \mathbb{M}_{D}^{n \times n}\right)$ the function $t \mapsto\left\langle\nu_{p}(t), \varphi\right\rangle$ is $\mu$-integrable and

$$
\langle p(b)-p(a)\rangle=\int_{a}^{b}\left\langle\nu_{p}(t), \varphi\right\rangle \mathrm{d} \mu(t)
$$

for every $a, b \in[0, T]$, with $a \leq b$, such that $\mu(\{a\})=\mu(\{b\})=0$. Moreover,

$$
\left\|\nu_{p}(t)\right\|_{1} \leq 1
$$

for $\mu$-a.e. $t \in[0, T]$.

Proposition 5.7. Let $(\alpha, u, e, p)$ be a quasistatic viscosity evolution with datum $w$. Then

$$
\mathcal{E}(\alpha(\tau), e(\tau))-\mathcal{E}\left(\alpha\left(\tau^{+}\right), e\left(\tau^{+}\right)\right) \geq 0
$$

for every $\tau \in S^{\circ} \cap[0, T)$, and

$$
\begin{aligned}
& \mathcal{E}(\alpha(T), e(T))+\lambda \int_{0}^{T} \mathcal{H}\left(\alpha(T), \nu_{p}(t)\right) \mathrm{d} \mu_{d}(t)+(1-\lambda) \int_{0}^{T} \mathcal{H}\left(\alpha(t), \nu_{p}(t)\right) \mathrm{d} \mu_{d}(t) \\
& +\sum_{\tau \in S^{\circ} \cap[0, T)}\left(\mathcal{E}(\alpha(\tau), e(\tau))-\mathcal{E}\left(\alpha\left(\tau^{+}\right), e\left(\tau^{+}\right)\right)\right)=\mathcal{E}\left(\alpha_{0}, e_{0}\right)+\int_{0}^{T}\langle\sigma(t), E \dot{w}(t)\rangle \mathrm{d} t .
\end{aligned}
$$

Proof. For every $\tau \in S^{\circ} \cap[0, T)$ evaluating the energy balance (ev4) in $\left(s_{-}^{\circ}(\tau), s_{+}^{\circ}(\tau)\right) \subset U^{\circ}$ gives, since $\dot{t}^{\circ}=0$ in $U^{\circ}$,

$$
\begin{aligned}
& \int_{s_{-}^{\circ}(\tau)}^{s_{+}^{\circ}(\tau)}\left(\lambda \mathcal{H}\left(\alpha^{\circ}\left(s_{-}^{\circ}(T)\right), \dot{p}^{\circ}(s)\right)+(1-\lambda) \mathcal{H}\left(\alpha^{\circ}(s), \dot{p}^{\circ}(s)\right)+\left\|\dot{\alpha}^{\circ}(s)\right\|_{2} \Psi\left(\alpha^{\circ}(s), e^{\circ}(s) ; p^{\circ}, s\right)\right) \mathrm{d} s \\
& \quad=\mathcal{E}\left(\alpha^{\circ}\left(s_{-}^{\circ}(\tau)\right), e^{\circ}\left(s_{-}^{\circ}(\tau)\right)\right)-\mathcal{E}\left(\alpha^{\circ}\left(s_{+}^{\circ}(\tau)\right), e^{\circ}\left(s_{+}^{\circ}(\tau)\right)\right) .
\end{aligned}
$$

By definition of quasistatic viscosity evolutions and (5.10), we get immediately (5.11). Moreover, arguing as in $[8$, Lemma 5.5] we deduce

$$
\begin{gathered}
\int_{\left(0, s_{-}^{\circ}(T)\right) \backslash U^{\circ}}\left(\lambda \mathcal{H}\left(\alpha^{\circ}\left(s_{-}^{\circ}(T)\right), \dot{p}^{\circ}(s)\right)+(1-\lambda) \mathcal{H}\left(\alpha^{\circ}(s), \dot{p}^{\circ}(s)\right)\right) \mathrm{d} s \\
=\int_{0}^{T}\left(\lambda \mathcal{H}\left(\alpha(T), \nu_{p}(t)\right)+(1-\lambda) \mathcal{H}\left(\alpha(t), \nu_{p}(t)\right)\right) \mathrm{d} \mu_{d}(t) .
\end{gathered}
$$

The energy balance $\left(\right.$ ev4) in $\left(0, s_{-}^{\circ}(T)\right)$ reads

$$
\begin{aligned}
& \mathcal{E}\left(\alpha^{\circ}\left(s_{-}^{\circ}(T)\right), e^{\circ}\left(s_{-}^{\circ}(T)\right)\right)+\lambda \int_{0}^{s_{-}^{\circ}(T)} \mathcal{H}\left(\alpha^{\circ}\left(s_{-}^{\circ}(T)\right), \dot{p}^{\circ}(s)\right) \mathrm{d} s+(1-\lambda) \int_{0}^{s_{-}^{\circ}(T)} \mathcal{H}\left(\alpha^{\circ}(s), \dot{p}^{\circ}(s)\right) \mathrm{d} s \\
& +\int_{\left(0, s_{-}^{\circ}(T)\right) \cap U^{\circ}}\left\|\dot{\alpha}^{\circ}(s)\right\|_{2} \Psi\left(\alpha^{\circ}(s), e^{\circ}(s) ; p^{\circ}, s\right) \mathrm{d} s=\mathcal{E}\left(\alpha_{0}, e_{0}\right)+\int_{0}^{s_{-}^{\circ}(T)}\left\langle\sigma^{\circ}(s), E \dot{w}^{\circ}(s)\right\rangle \mathrm{d} s,
\end{aligned}
$$

hence we deduce (5.12) from (5.13) and (5.14), recalling (4.13) and the definition of quasistatic viscosity evolution.

Remark 5.8. Neglecting the positive viscous terms in (5.12) an energy inequality can be written in every subinterval $\left[t_{1}, t_{2}\right]$ of $[0, T]$. This inequality holds as an equality, also with $\mu_{d}=\mu$, in every subinterval $\left[t_{1}, t_{2}\right]$ such that $\left[t_{1}, t_{2}\right] \cap S^{\circ}=\emptyset$, with $S^{\circ}$ introduced in (4.12). 


\section{A. Auxiliary Results}

We collect in this appendix two abstract results used throughout the paper.

First we prove a compactness result used to construct $\varepsilon$-approximate viscous evolutions in Section 3 . If $X$ is a reflexive space it is well known that $L^{2}(0, T ; X)$ is isomorphic to the dual space of $L^{2}\left(0, T ; X^{\prime}\right)$, where $X^{\prime}$ is the dual space of $X$. We now consider the case when $X$ is only the dual of a separable Banach space $Y$ : every function in $L^{2}(0, T ; X)$ is in the dual of $L^{2}(0, T ; Y)$ but the limit (in the sense of the dual of $L^{2}(0, T ; Y)$ ) of a converging sequence in $L^{2}(0, T ; X)$ could be weakly* measurable but not strongly measurable.

A function $f:(0, T) \rightarrow X$ is said weakly* measurable if $(0, T) \ni t \mapsto\langle f(t), g\rangle$ is measurable for every $g \in Y$. Let us denote

$$
L_{w}^{2}(0, T ; X):=\left\{p:[0, T] \rightarrow X \text { weakly* measurable }: t \mapsto\|p(t)\| \in L^{2}(0, T)\right\} .
$$

Adapting the proof of [39, Theorem IV.1.8] we can see that there is an algebraic isomorphism $\mathcal{I}$ between the dual space of $L^{2}(0, T ; Y)$ and $L_{w}^{2}(0, T ; X)$ given, for every $p \in L_{w}^{2}(0, T ; X)$ and $\varphi \in L^{2}(0, T ; Y)$, by

$$
\mathcal{I}(p)(\varphi):=\int_{0}^{T}\langle p(t), \varphi(t)\rangle \mathrm{d} t, \text { with }\|\mathcal{I}(p)\|^{2}=\int_{0}^{T}\|p(t)\|^{2} \mathrm{~d} t
$$

This defines the weak ${ }^{*}$ convergence in $L_{w}^{2}(0, T ; X)$. In the following we study the space of functions with distributional time derivative in $L_{w}^{2}(0, T ; X)$. In Section 3 the lemma below is applied to the case of $X=M_{b}\left(\Omega \cup \partial_{D} \Omega ; \mathbb{M}_{D}^{n \times n}\right)$ and $Y=C_{0}\left(\Omega \cup \partial_{D} \Omega ; \mathbb{M}_{D}^{n \times n}\right)$. Notice that $Y$ can be identified with the space of functions in $C\left(\bar{\Omega} ; \mathbb{M}_{D}^{n \times n}\right)$ vanishing on $\overline{\partial_{N} \Omega}$.

Lemma A.1. Let $X$ be the dual space of a separable Banach space $Y$ and let

$$
\begin{gathered}
H_{w}^{1}(0, T ; X):=\left\{p \in L_{w}^{2}(0, T ; X): \exists \widetilde{p} \in L_{w}^{2}(0, T ; X) \text { s.t. for every } \varphi \in C_{c}^{1}((0, T) ; Y)\right. \\
\left.\int_{0}^{T}\left\langle p(t), \partial_{t} \varphi(t)\right\rangle \mathrm{d} t=-\int_{0}^{T}\langle\widetilde{p}(t), \varphi(t)\rangle \mathrm{d} t\right\} .
\end{gathered}
$$

Then every $p \in H_{w}^{1}(0, T ; X)$ admits a unique representative absolutely continuous into $X$, its distributional derivative $\widetilde{p}$ is characterized by

$$
\widetilde{p}(t)=w^{*}-\lim _{s \rightarrow t} \frac{p(s)-p(t)}{s-t}=: \dot{p}(t) \text { for a.e. } t \in(0, T),
$$

and

$$
\|p\|_{C^{0,1 / 2}([0, T] ; X)} \leq C\left(\|p(\cdot)\|_{2}+\|\dot{p}(\cdot)\|_{2}\right),
$$

with $C$ independent of $p \in H_{w}^{1}(0, T ; X)$.

Moreover, for every sequence $\left\{p_{k}\right\}_{k} \subset H_{w}^{1}(0, T ; X)$ with $\left\|p_{k}(\cdot)\right\|_{2}+\left\|\dot{p}_{k}(\cdot)\right\|_{2} \leq C$ for every $k$, there exists a function $p \in H_{w}^{1}(0, T ; X)$ such that, up to a subsequence,

$$
p_{k}(t) \stackrel{*}{\rightarrow} p(t) \text { weakly* } \text { in } X \text { for every } t \in[0, T], \quad \dot{p}_{k} \stackrel{*}{\rightarrow} \dot{p} \text { weakly* in } L_{w}^{2}(0, T ; X) .
$$

Proof. Let $\rho$ be the standard mollifier in $\mathbb{R}$ and $\rho_{k}(t):=k \rho\left(\frac{t}{k}\right)$. For every $t_{1} \leq t_{2} \in[0, T], \psi \in Y$, we take in (A.1) $\varphi_{k}(t)=\psi \omega_{k}(t)$, where $\omega_{k}$ is the convolution product between $\rho_{k}$ and the indicator function of $\left[t_{1}, t_{2}\right]$, and let $k$ tend to $+\infty$. Then we get that for every $p \in H_{w}^{1}(0, T ; X)$

$$
\left\langle p\left(t_{2}\right)-p\left(t_{1}\right), \psi\right\rangle=\int_{t_{1}}^{t_{2}}\langle\widetilde{p}(s), \psi\rangle \mathrm{d} s .
$$

Since $\int_{t_{1}}^{t_{2}}\langle\widetilde{p}(s), \psi\rangle \mathrm{d} s \leq \int_{t_{1}}^{t_{2}}\|\widetilde{p}(s)\| \mathrm{d} s$ for every $\|\psi\| \leq 1$, it follows that

$$
\left\|p\left(t_{2}\right)-p\left(t_{1}\right)\right\| \leq \int_{t_{1}}^{t_{2}}\|\widetilde{p}(s)\| \mathrm{d} s,
$$

and then $p$ is absolutely continuous, $s \mapsto\|\widetilde{p}(s)\|$ being in $L^{2}$. Then [5, Lemma 7.1] implies that for a.e. $t \in(0, T)$ the weak* limit $\dot{p}(t)$ defined in (A.2) exists. Let us now consider the function $h(t):=\|p(t)\|$ : we have

$$
|h(t)-h(s)| \leq\|p(t)-p(s)\|,
$$

and therefore, by (A.5) and the Hölder inequality, $h \in H^{1}(0, T)$ and $|\dot{h}(t)| \leq\|\dot{p}(t)\|$ for a.e. $t \in(0, T)$. From the Sobolev embedding theorem for real valued functions (A.3) follows.

By (A.4) and a standard argument that uses the separability of $Y$, we obtain that for a.e. $t \in(0, T)$ it holds

and then (A.2) follows.

$$
\lim _{s \rightarrow t}\left\langle\frac{p(s)-p(t)}{s-t}, \psi\right\rangle=\langle\widetilde{p}(t), \psi\rangle \text { for every } \psi \in Y,
$$


By (A.3), every sequence $\left\{p_{k}\right\}_{k}$ as in the statement is equibounded in $C^{0,1 / 2}([0, T] ; X)$, and in particular $\left\|p_{k}(t)\right\| \leq M$ for every $k$ and $t$. It is now well known that, since $Y$ is separable, there exists a distance $\mathrm{d}_{M}$ on $B_{M}$, the ball of $X$ with radius $M$ centered in the origin, inducing the weak* convergence, and the metric space $\left(B_{M}, \mathrm{~d}_{M}\right)$ is complete. Then the Arzelà-Ascoli Theorem implies that there exists $p \in C^{0,1 / 2}([0, T] ; X)$ such that, up to a subsequence,

$$
p_{k}(t) \stackrel{*}{\rightarrow} p(t) \text { in } X, \text { for every } t \in[0, T] .
$$

Since $\left\|\dot{p}_{k}(\cdot)\right\|_{2} \leq C$, there exists $\widehat{p} \in L_{w}^{2}(0, T ; X)$ such that, up to a subsequence,

$$
\dot{p}_{k} \rightarrow \widehat{p} \text { weakly* in } L_{w}^{2}(0, T ; X) \text {. }
$$

This implies that for every $\varphi \in C_{c}^{\infty}((0, T) ; Y)$

$$
\int_{0}^{T}\langle\widehat{p}(t), \varphi(t)\rangle \mathrm{d} t=-\int_{0}^{T}\left\langle p(t), \partial_{t} \varphi(t)\right\rangle \mathrm{d} t
$$

and therefore $\widehat{p}=\dot{p}$. This concludes the proof.

The following lemma is a generalization of the Riesz Representation Theorem for bounded linear functionals acting on the space of continuous functions. It is employed in Lemma 3.4.

Lemma A.2. Let $B$ be an open bounded subset of $\mathbb{R}^{n}$, and let $S$ be a distribution on $B$ such that

$$
\langle S, \beta\rangle \leq C\|\beta\|_{p} \quad \text { for every } \beta \in C_{c}^{\infty}(B),
$$

with $C>0$ and $p \in[1, \infty)$. Then there exists a unique pair $(g, \mu)$ such that $g \in L^{p^{\prime}}(B)$, with $\frac{1}{p^{\prime}}+\frac{1}{p}=1$, $g \geq 0, \mu \in M^{+}(B)$ (namely $\mu$ is a nonnegative measure on $B$ ), $g \mathrm{~d} x$ and $\mu$ are mutually singular, and

$$
\langle S, \beta\rangle=\int_{B} g \beta \mathrm{d} x-\int_{B} \beta \mathrm{d} \mu \quad \text { for every } \beta \in C_{c}^{\infty}(B) .
$$

Proof. In the following we will use the notation $C_{0}^{+}(B):=\left\{\beta \in C_{0}(B): \beta \geq 0\right\}$, and the analogous for $C_{0}^{-}(B)$.

Recall that every $\beta \in C_{0}^{+}(B)$ can be approximated uniformly (and thus in $L^{p}$-norm) from below in $C_{c}^{\infty}(B) \cap C_{0}^{+}(B)$. We define

$$
\left\langle S^{+}, \beta\right\rangle:=\sup _{\substack{\varphi \in C_{c}^{\infty}(B) \\ 0 \leq \varphi \leq \beta}}\langle S, \varphi\rangle \quad \text { for every } \beta \in C_{0}^{+}(B),
$$

which satisfies

$$
0 \leq\left\langle S^{+}, \beta\right\rangle \leq C\|\beta\|_{p}
$$

for every $\beta \in C_{0}^{+}(B)$ by (A.6). Following [31, Proposition 24], we extend $S^{+}$by setting

$$
\left\langle S^{+}, \beta\right\rangle:=-\left\langle S^{+},-\beta\right\rangle \text { for every } \beta \in C_{0}^{-}(B)
$$

and we see that the functional $S^{+}$is linear and positive on $C_{0}(B)$. Moreover

$$
\left|\left\langle S^{+}, \beta\right\rangle\right|=\left|\left\langle S^{+}, \beta^{+}\right\rangle-\left\langle S^{+}, \beta^{-}\right\rangle\right| \leq 2 C\|\beta\|_{p} \quad \text { for every } \beta \in C_{0}(B),
$$

and thus there exists $g \in L^{p^{\prime}}(B)$ such that

$$
\left\langle S^{+}, \beta\right\rangle=\int_{B} g \beta \mathrm{d} x \quad \text { for every } \beta \in C_{0}(B) .
$$

Since $\left\langle S^{+}, \beta\right\rangle \in \mathbb{R}$ for every $\beta$, the distribution

$$
\left\langle S^{-}, \beta\right\rangle:=\left\langle S^{+}, \beta\right\rangle-\langle S, \beta\rangle \quad \text { for every } \beta \in C_{c}^{\infty}(B)
$$

is well defined and by (A.8) we obtain

$$
\left\langle S^{-}, \beta\right\rangle \geq 0 \quad \text { for every } C_{c}^{\infty}(B) \cap C_{0}^{+}(B) .
$$

It is well known from the theory of distributions that there exists a nonnegative measure $\mu \in M^{+}(B)$ such that

$$
\left\langle S^{-}, \beta\right\rangle=\int_{B} \beta \mathrm{d} \mu \text { for every } C_{c}^{\infty}(B) .
$$

Collecting (A.9), (A.10), and (A.11) we find that $g$ and $\mu$ satisfy the properties as in the statement. Since every measure is uniquely decomposed into a nonnegative and a nonpositive part, the uniqueness of $g$ and $\mu$ follows. Thus the proof is concluded. 
Acknowledgments. The authors thank Gianni Dal Maso for discussions and for several helpful suggestions. V.C. received support from the PRIN 2010-11 grant for the project Calculus of Variations of the Italian Ministry of Education, University, and Research, as well as from the GNAMPA (Gruppo Nazionale per l'Analisi Matematica, la Probabilità e le loro Applicazioni) of INdAM (Istituto Nazionale di Alta Matematica). The work of G.L. is funded by the European Research Council through the Advanced Grant Quasistatic and Dynamic Evolution Problems in Plasticity and Fracture (290888).

\section{REFERENCES}

[1] R. Alessi, J.-J. Marigo, And S. Vidoli, Gradient damage models coupled with plasticity and nucleation of cohesive cracks, Arch. Ration. Mech. Anal., 214 (2014), pp. 575-615.

[2] R. Alessi, J.-J. Marigo, AND S. Vidoli, Gradient damage models coupled with plasticity: Variational formulation and main properties, Mechanics of Materials, 80, Part B (2015), pp. $351-367$.

[3] L. Ambrosio, N. Fusco, and D. Pallara, Functions of bounded variation and free discontinuity problems, Oxford Mathematical Monographs, The Clarendon Press, Oxford University Press, New York, 2000.

[4] V. CRismale, Globally stable quasistatic evolution for a coupled elastoplastic-damage model. Preprint SISSA 34/2014/MATE, 2014.

[5] G. Dal Maso, A. DeSimone, and M. G. Mora, Quasistatic evolution problems for linearly elastic-perfectly plastic materials, Arch. Ration. Mech. Anal., 180 (2006), pp. 237-291.

[6] G. Dal Maso, A. DeSimone, M. G. Mora, and M. Morini, Globally stable quasistatic evolution in plasticity with softening, Netw. Heterog. Media, 3 (2008), pp. 567-614.

[7] G. Dal Maso, A. DeSimone, and F. Solombrino, Quasistatic evolution for Cam-Clay plasticity: a weak formulation via viscoplastic regularization and time rescaling, Calc. Var. Partial Differ. Equ., 40 (2011), pp. 125-181.

[8] - Quasistatic evolution for Cam-Clay plasticity: properties of the viscosity solution, Calc. Var. Partial Differential Equations, 44 (2012), pp. 495-541.

[9] A. Demyanov, Regularity of stresses in Prandtl-Reuss perfect plasticity, Calc. Var. Partial Differential Equations, 34 (2009), pp. 23-72.

[10] M. A. EFendiev And A. Mielke, On the rate-independent limit of systems with dry friction and small viscosity, J. Convex Anal., 13 (2006), pp. 151-167.

[11] G. A. Francfort and A. Garroni, A variational view of partial brittle damage evolution, Arch. Ration. Mech. Anal., 182 (2006), pp. 125-152.

[12] G. A. Francfort and A. Giacomini, Small-strain heterogeneous elastoplasticity revisited, Comm. Pure Appl. Math., 65 (2012), pp. 1185-1241.

[13] M. Frémond, Non-smooth thermomechanics, Springer-Verlag, Berlin, 2002.

[14] C. Goffman and J. Serrin, Sublinear functions of measures and variational integrals, Duke Math. J., 31 (1964), pp. $159-178$.

[15] A. Ibrahimbegovic, Nonlinear solid mechanics, vol. 160 of Solid Mechanics and its Applications, Springer, Dordrecht, 2009.

[16] D. Knees, A. Mielke, And C. Zanini, On the inviscid limit of a model for crack propagation, Math. Models Methods Appl. Sci., 18 (2008), pp. 1529-1569.

[17] D. Knees, R. Rossi, and C. ZAnini, A quasilinear differential inclusion for viscous and rate-independent damage systems in non-smooth domains. WIAS Preprint No. 1867, 2013.

[18] - A vanishing viscosity approach to a rate-independent damage model, Math. Models Methods Appl. Sci., 23 (2013), pp. 565-616.

[19] R. Kohn and R. Temam, Dual spaces of stresses and strains, with applications to Hencky plasticity, Appl. Math. Optim., 10 (1983), pp. 1-35.

[20] P. KREJČí, Evolution variational inequalities and multidimensional hysteresis operators, in Nonlinear differential equations (Chvalatice, 1998), vol. 404 of Chapman \& Hall/CRC Res. Notes Math., Chapman \& Hall/CRC, Boca Raton, FL, 1999, pp. 47-110.

[21] G. Lazzaroni and R. Toader, A model for crack propagation based on viscous approximation, Math. Models Methods Appl. Sci., 21 (2011), pp. 2019-2047.

[22] J. Lemaitre and J. Chabouche, Mechanics of Solid Materials, Cambridge University Press, Avon, 1990.

[23] H. Matthies, G. Strang, and E. Christiansen, The saddle point of a differential program, in Energy Methods in Finite Element Analysis, Z. O. Glowinski R., Rodin E., ed., Wiley, New York, 1979, pp. 309-318.

[24] A. Mielke, Evolution of rate-independent systems, in Evolutionary equations. Vol. II, Handb. Differ. Equ., Elsevier/North-Holland, Amsterdam, 2005, pp. 461-559.

[25] A. Mielke, R. Rossi, AND G. SAVARÉ, BV solutions and viscosity approximations of rate-independent systems, ESAIM Control Optim. Calc. Var., 18 (2012), pp. 36-80.

[26] — Balanced viscosity (BV) solutions to infinite-dimensional rate-independent systems. to appear on J. Eur. Math. Soc., 2015.

[27] A. Mielke And T. Roubíček, Rate-independent damage processes in nonlinear elasticity, Math. Models Methods Appl. Sci., 16 (2006), pp. 177-209.

[28] M. NeGRI, Quasi-static rate-independent evolutions: characterization, existence, approximation and application to fracture mechanics, ESAIM Control Optim. Calc. Var., 20 (2014), pp. 983-1008.

[29] C. G. Panagiotopoulos, V. Mantič, and T. RoubÍČEK, BEM solution of delamination problems using an interface damage and plasticity model, Comput. Mech., 51 (2013), pp. 505-521.

[30] T. Roubíček, M. KRužík, And J. Zeman, Delamination and adhesive contact models and their mathematical analysis and numerical treatment, in Math. Methods and Models in Composites. Vol. 5, V. Mantič, ed., Comput. Experiment. Meth. in Structures, Imperial College Press, 2014, pp. 349-400.

[31] H. L. Royden, Real analysis, Macmillan Publishing Company, New York, third ed., 1988.

[32] W. Rudin, Real and Complex Analysis, McGraw-Hill, New York, 1966. 
[33] F. Solombrino, Quasistatic evolution problems for nonhomogeneous elastic plastic materials, J. Convex Anal., 16 (2009), pp. 89-119.

[34] $ـ$ A-priori time regularity estimates and a simplified proof of existence in perfect plasticity, Mathematische Nachrichten, (2015).

[35] P.-M. Suquet, Sur les équations de la plasticité: existence et régularité des solutions, J. Mécanique, 20 (1981), pp. 3-39.

[36] R. Temam, Mathematical problems in plasticity, Gauthier-Villars, Paris, 1985. Translation of Problèmes mathématiques en plasticité. Gauthier-Villars, Paris, 1983.

[37] R. Temam and G. Strang, Duality and relaxation in the variational problem of plasticity, J. Mécanique, 19 (1980), pp. $493-527$.

[38] M. Thomas and A. Mielke, Damage of nonlinearly elastic materials at small strain-existence and regularity results, ZAMM Z. Angew. Math. Mech., 90 (2010), pp. 88-112.

[39] J. WARGA, Optimal control of differential and functional equations, Academic Press, New York-London, 1972.

SisSA, via Bonomea 265, 34136 Trieste, Italy

E-mail address, V. Crismale: vito.crismale@sissa.it

E-mail address, G. Lazzaroni: giuliano.lazzaroni@sissa.it 